\title{
THE HODGE THEORY OF ALGEBRAIC MAPS
}

\author{
By MARK ANDREA A. DE CATALDO ${ }^{1}$ AND LUCA MIGLIORINI ${ }^{2}$
}

ABSTRACT. - We give a geometric proof of the Decomposition Theorem of Beilinson, Bernstein, Deligne and Gabber for the direct image of the intersection cohomology complex under a proper map of complex algebraic varieties. The method rests on new Hodge-theoretic results on the cohomology of projective varieties which extend naturally the classical theory and provide new applications.

() 2005 Elsevier SAS

RÉSUMÉ. - On donne une démonstration géométrique du théorème de décomposition de Beilinson, Bernstein, Deligne et Gabber pour l'image directe, par un morphisme propre de variétés algébriques complexes, du complexe de cohomologie d'intersection. La preuve s'appuie sur des résultats nouveaux concernant la théorie de Hodge des variétés projectives, qui généralisent la théorie classique et donnent de nouvelles applications.

(C) 2005 Elsevier SAS

\section{Contents}

1 Introduction . . . . . . . . . . . . . . . . . . . . . . . . . . . . . . . .694

2 Statements .........................................698

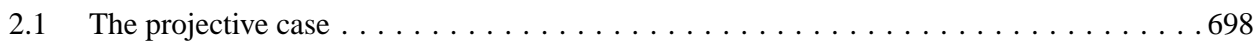

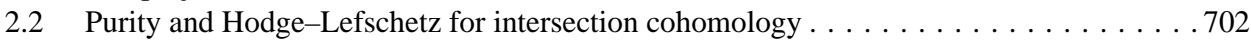

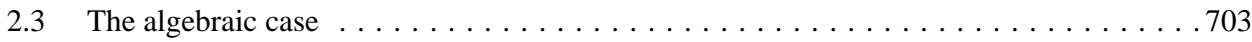

2.4 Example: Resolution of singularities of a threefold $\ldots \ldots \ldots \ldots \ldots \ldots \ldots \ldots$

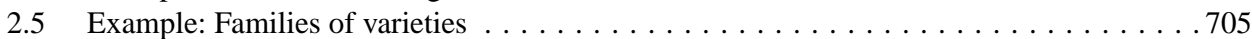

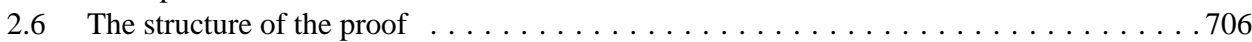

3 Notation and preliminary results $\ldots \ldots \ldots \ldots \ldots \ldots \ldots \ldots \ldots \ldots$

3.1 The topology and Hodge Theory of algebraic varieties $\ldots \ldots \ldots \ldots \ldots \ldots$

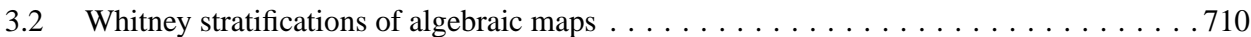

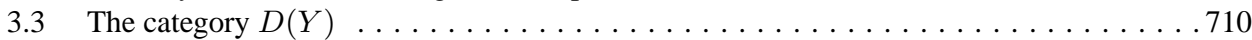

3.4 The intersection form on the fibers of the $\operatorname{map} f: X \rightarrow Y \ldots \ldots \ldots \ldots \ldots \ldots 11$

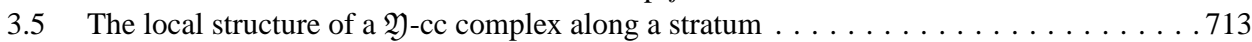

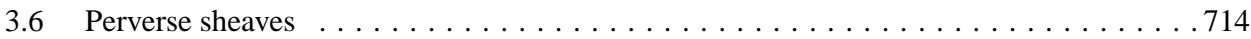

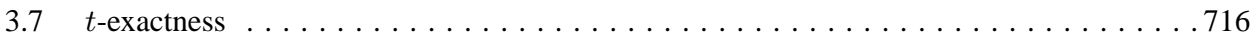

3.8 Intersection cohomology complexes, semisimple objects and intermediate extensions . . 716

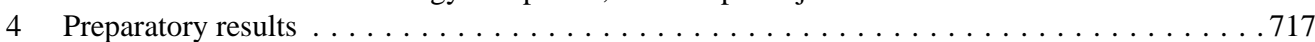

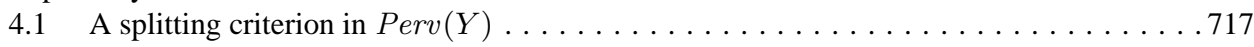

4.2 The perverse filtration . . . . . . . . . . . . . . . . . . . . 719

\footnotetext{
${ }^{1}$ Partially supported by NSF Grant DMS 0202321, NSA Grant MDA904-02-1-0100.

${ }^{2}$ Partially supported by GNSAGA.
} 


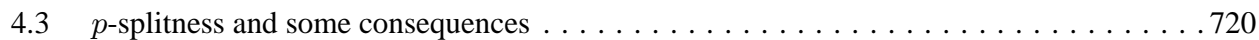

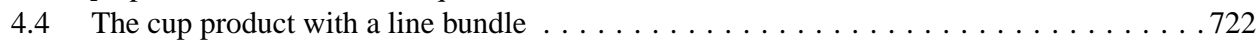

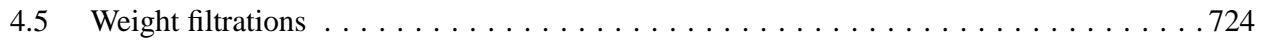

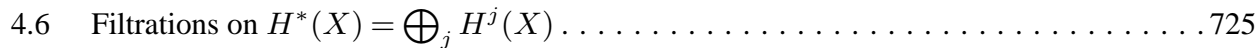

4.7 The Universal Hyperplane section and the defect of semismallness . . . . . . . . . 726

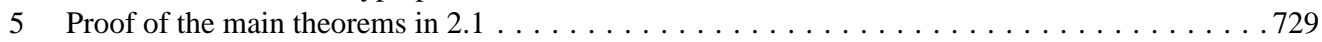

5.1 Relative Hard Lefschetz, Decomposition and Semisimplicity $(i \neq 0) \ldots \ldots$. . . . . . 729

5.2 Hard Lefschetz for perverse cohomology groups . . . . . . . . . . . . . . . 730

5.3 The Generalized Hodge-Riemann Bilinear Relations $\left(P_{j}^{i} \neq P_{0}^{0}\right) \ldots \ldots \ldots \ldots \ldots$

5.4 The space $\Lambda \subseteq H^{n}(X)$, its approximability and the polarization of $P_{0}^{0} \ldots \ldots \ldots 734$

6 The Semisimplicity Theorem $2.1 .1(\mathrm{c})$ for ${ }^{p} \mathcal{H}^{0}\left(f_{*} \mathbb{Q}_{X}[n]\right) \ldots \ldots \ldots \ldots \ldots \ldots \ldots 737$

6.1 The induction on the strata: reduction to $S_{0} \ldots \ldots \ldots \ldots \ldots \ldots \ldots \ldots \ldots \ldots 737$

6.2 The local system $\mathcal{H}^{-s}\left(\alpha_{s}^{!}{ }^{p} \mathcal{H}^{0}\left(f_{*} \mathbb{Q}_{X}[n]\right)\right)$ on $S_{s} \ldots \ldots \ldots \ldots \ldots \ldots \ldots$

$6.3{ }^{p} \mathcal{H}^{0}\left(f_{*} \mathbb{Q}_{X}[n]\right)$ is a direct sum of intersection cohomology complexes . . . . . . .740

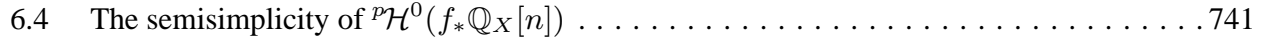

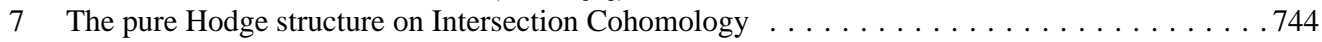

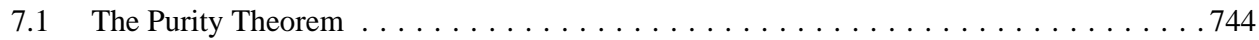

7.2 The Hodge-Lefschetz Theorem . . . . . . . . . . . . . . . . . . . . . . . 747

Acknowledgements . . . . . . . . . . . . . . . . . . . . . . . . . . . .748

References . . . . . . . . . . . . . . . . . . . . . . . . . . . 749

\section{Introduction}

Let $f: X \rightarrow Y$ be a proper map of complex algebraic varieties, $n=\operatorname{dim} X$. For ease of exposition only, assume that $X$ is nonsingular and that $X$ and $Y$ are projective. Let $\eta$ and $A$ be ample line bundles on $X$ and $Y$ respectively, and set $L:=f^{*} A$.

If $f$ is a smooth family, then the classical Hard Lefschetz Theorem for $\eta$ applied to the fibers of $f$ gives isomorphisms for every $i \geqslant 0$ :

$$
\eta^{i}: R^{n-\operatorname{dim} Y-i} f_{*} \mathbb{Q}_{X} \simeq R^{n-\operatorname{dim} Y+i} f_{*} \mathbb{Q}_{X}
$$

which give rise to a direct sum decomposition for the direct image complex

$$
R f_{*} \mathbb{Q}_{X} \simeq \bigoplus_{i} R^{i} f_{*} \mathbb{Q}_{X}[-i]
$$

in the derived category of the category of sheaves on $Y$ (cf. [9]). This important fact implies, for example, the $E_{2}$-degeneration of the Leray spectral sequence for $f$. The sheaves $R^{i} f_{*} \mathbb{Q}_{X}$ are semisimple local systems, i.e. they split as a direct sum of local systems with no nontrivial local subsystems. Note that the category of finite dimensional local systems is abelian, noetherian and artinian.

At first sight, nothing similar happens for an arbitrary map $f: X \rightarrow Y$. The isomorphisms (1) and (2) fail in general, the Leray spectral sequence may not degenerate at $E_{2}$ and the abelian category of sheaves on $Y$ is neither noetherian, nor artinian.

The Leray spectral sequence is associated with the "filtration" of $R f_{*} \mathbb{Q}_{X}$ by the truncated complexes $\tau_{\leqslant i} R f_{*} \mathbb{Q}_{X}$. The $i$-th direct image $R^{i} f_{*} \mathbb{Q}_{X}$ appears, up to a shift, as the cone of the natural map $\tau_{\leqslant i-1} R f_{*} \mathbb{Q}_{X} \rightarrow \tau_{\leqslant i} R f_{*} \mathbb{Q}_{X}$, i.e. as the $i$-th cohomology sheaf of the complex $R f_{*} \mathbb{Q}_{X}$.

One of the main ideas leading to the theory of perverse sheaves in [1] is that all the facts mentioned in the case of a smooth family hold for an arbitrary map, provided that they are re-formulated with respect to a notion of truncation different from the one leading to

$4^{\mathrm{e}}$ SÉRIE - TOME $38-2005-\mathrm{N}^{\circ} 5$ 
the cohomology sheaves, that is with respect to the so-called perverse truncation ${ }^{p} \tau_{\leqslant i}$, and that we replace the sheaves $R^{i} f_{*} \mathbb{Q}_{X}$ with the shifted cones ${ }^{p} \mathcal{H}^{i}\left(R f_{*} \mathbb{Q}_{X}\right)$ of the mappings $p_{\tau_{\leqslant i-1}} R f_{*} \mathbb{Q}_{X} \rightarrow{ }^{p} \tau_{\leqslant i} R f_{*} \mathbb{Q}_{X}$. These cones are called the perverse cohomology of $R f_{*} \mathbb{Q}_{X}$ and are perverse sheaves. Despite their name, perverse sheaves are complexes in the derived category of the category of sheaves on $Y$ which are characterized by conditions on their cohomology sheaves. Just like local systems, the category of perverse sheaves is abelian, noetherian and artinian. Its simple objects are the intersection cohomology complexes of simple local systems on strata. Whenever $Y$ is nonsingular and the stratification is trivial, perverse sheaves are, up to a shift, just local systems.

That these notions are the correct generalization to arbitrary proper morphisms of the situation considered above for smooth morphisms, is shown by the beautiful Relative Hard Lefschetz Theorem and Decomposition Theorem, proved in [1] by Beilinson, Bernstein and Deligne using algebraic geometry in positive characteristic. They generalize the isomorphisms (1) and (2) for a smooth family to the case of an arbitrary projective map from an algebraic manifold: the map induced by the line bundle $\eta$ in perverse cohomology

$$
\eta^{i}:{ }^{p} \mathcal{H}^{-i}\left(R f_{*} \mathbb{Q}_{X}[n]\right) \rightarrow{ }^{p} \mathcal{H}^{i}\left(R f_{*} \mathbb{Q}_{X}[n]\right)
$$

is an isomorphism for every $i \geqslant 0$ and we have a direct sum decomposition

$$
R f_{*} \mathbb{Q}_{X}[n] \simeq \bigoplus_{i}^{p} \mathcal{H}^{i}\left(R f_{*} \mathbb{Q}_{X}[n]\right)[-i] .
$$

As a consequence, the so-called perverse Leray spectral sequence $\mathbb{H}^{l}\left(Y,{ }^{p} \mathcal{H}^{m}\left(R f_{*} \mathbb{Q}_{X}[n]\right)\right) \Longrightarrow$ $H^{n+l+m}(X, \mathbb{Q})$ is $E_{2}$-degenerate. This fact alone has striking computational and theoretical consequences. For example, the intersection cohomology groups of a variety $Y$ inject in the ordinary singular cohomology groups of any resolution $X$ of the singularities of $Y$. The semisimplicity statement for the local systems $R^{i} f_{*} \mathbb{Q}_{X}$ has a far-reaching generalization in the Semisimplicity Theorem, also proved in [1]: there is a canonical isomorphism of perverse sheaves

$$
{ }^{p} \mathcal{H}^{i}\left(R f_{*} \mathbb{Q}_{X}[n]\right) \simeq \bigoplus_{l} I C_{\overline{S_{l}}}\left(L_{i, l}\right)
$$

where the $I C_{\overline{S_{l}}}\left(L_{i, l}\right)$ are the Goresky-MacPherson intersection cohomology complexes on $Y$ associated with certain semisimple local systems $L_{i, l}$ on the strata of a finite algebraic stratification $Y=\coprod_{l=0}^{\operatorname{dim} Y} S_{l}$ for the map $f$.

Analogous results hold for a possibly singular $X$, provided one replaces $R f_{*} \mathbb{Q}_{X}[n]$ by the intersection cohomology complex $I C_{X}$.

These three theorems are cornerstones of the topology of algebraic maps. They have found many applications to algebraic geometry and to representation theory and, in our opinion, should be regarded as expressing fundamental properties of complex algebraic geometry.

In our previous paper [6] we proved that if $f$ is semismall, then $L$ behaves Hodgetheoretically like an ample line bundle: the Hard Lefschetz Theorem holds for $L$ acting on rational cohomology, i.e. $L^{r}: H^{n-r}(X) \simeq H^{n+r}(X)$, for every $r$, and the primitive subspaces $\operatorname{Ker} L^{r+1} \subseteq H^{n-r}(X)$ are polarized by means of the intersection form on $X$. Associated with a stratification of the map $f$ there is a series of intersection forms describing how the fiber of a point in a given stratum intersects in $X$ the pre-image of the stratum. In the case of a semismall map there is only one intersection form for each stratum component. The discovery of the above polarizations, joined with an argument of mixed Hodge structures showing that for 
every $y \in Y$ the group $H_{n}\left(f^{-1}(y)\right)$ injects in $H^{n}(X)$, allowed us to prove that the intersection forms are definite and thus nondegenerate. This generalizes the well-known result of Grauert for the contraction of curves on surfaces. By means of an induction on the strata, the statement of the semisimplicity theorem (5) for semismall maps $f$ was proved to be equivalent to the fact that the intersection forms are nondegenerate. The statements (3) and (4) are trivial for semismall maps since $R f_{*} \mathbb{Q}_{X}[n] \simeq{ }^{p} \mathcal{H}^{0}\left(R f_{*} \mathbb{Q}_{X}[n]\right)$. Our result about the intersection forms being definite can be seen as a "Decomposition Theorem with signs for semismall maps," i.e. as a polarized version of this theorem.

In this paper, in the spirit of our paper [6], we give a geometric proof of the Relative Hard Lefschetz, Decomposition and Semisimplicity isomorphisms (3)-(5). We complement these results by uncovering a series of Hodge-theoretic properties of the singular rational cohomology groups $H^{*}(X)$ and of the natural maps $H_{n-*}\left(f^{-1}(y)\right) \rightarrow H^{n+*}(X), y \in Y$.

We now discuss our results. By standard reductions, most statements remain valid in the context of proper maps of algebraic varieties (cf. 2.3).

The perverse truncation, which is defined locally over $Y$ by means of the topological operations of push-forward and truncation with respect to a stratification for $f$, gives rise to an increasing filtration $H_{\leqslant b}^{l}(X) \subseteq H^{l}(X)$ and to the corresponding graded perverse cohomology groups $H_{b}^{l}(X)=H_{\leqslant b}^{l}(X) / H_{\leqslant b-1}^{l}(X)$. See 4.2.

Our first result is the Hard Lefschetz Theorem for Perverse Cohomology Groups 2.1.4. While the Hard Lefschetz Theorem for the pull-back line bundle $L$ acting on ordinary cohomology fails, due to the lack of positivity of $L$ on the fibers of the map $f$, the analogous result, with a natural shift in cohomological degree, holds for the perverse cohomology groups.

It is as if the perverse filtration were calibrated precisely for the purpose of correcting the failure of the Hard Lefschetz Theorem for L. In fact, this result implies that the perverse filtration coincides with the canonical weight filtration associated with the Jordan form of the nilpotent operator $L$ acting on $H^{*}(X)$ (cf. 5.2.4). The results we find exhibit a striking similarity with the structures discovered on the cohomology of the limit fiber by Cattani, Kaplan, Schmid [4] and Kashiwara, Kawai [20], in the case of degenerating families along a normal crossing divisor, where the logarithms of the monodromies, instead of the cup products with Chern classes of line bundles, are the endomorphisms giving the filtrations. We find this aspect of our approach quite intriguing.

Since the weight filtration above, being characterized in terms of the $(1,1)$-operator $L$, is automatically Hodge-theoretic, we get the Hodge Structure Theorem 2.1.5 stating that the perverse filtration, and hence the perverse cohomology groups, are endowed with canonical Hodge structures.

The Hard Lefschetz Theorem for perverse cohomology groups implies the $(\eta, L)$-Decomposition Theorem 2.1.7, i.e. a Lefschetz-type direct sum decomposition of the perverse cohomology groups of $X$ into " $(\eta, L)$-primitive" Hodge sub-structures. The decomposition is orthogonal with respect to certain polarizing bilinear forms $S^{\eta L}$ coming from the Poincaré pairing on $X$ modified by $L$ and $\eta$.

The Generalized Hodge-Riemann Bilinear Relations Theorem 2.1.8 states that the forms $S^{\eta L}$ polarize up to sign the $(\eta, L)$-primitive spaces.

In our approach, it is crucial to describe (cf. 5.4) the subspace of cohomology classes of $H^{n}(X)$ which are limits for $\varepsilon \rightarrow 0^{+}$of cohomology classes primitive with respect to the ample line bundles of the form $L+\varepsilon \eta$.

The Generalized Grauert Contractibility Criterion 2.1.9 and the Refined Intersection Form Theorem 2.1.10 establish some of the Hodge-theoretic properties of the homology groups $H_{*}\left(f^{-1}(y)\right)$ and of the refined intersection forms defined on them (cf. 3.4).

$4^{\text {e }}$ SÉRIE - TOME $38-2005-\mathrm{N}^{\circ} 5$ 
To our knowledge, this rich structure on $H^{*}(X)$ and on $H^{*}\left(f^{-1}(y)\right)$ has not been spelled-out before and it should have significant geometric applications. We discuss two examples in 2.4 and 2.5 .

We propose two applications, the Contractibility Criterion 2.1 .12 and the Signature for Semismall Maps Theorem 2.1.14.

In our approach, not only are these structures and results complementary to the Decomposition Theorem, but they are also instrumental in proving it. In view of the inductive approach we develop in 6.1.3, the Decomposition Theorem implies that the refined intersection forms (more precisely, one of the graded parts) are nondegenerate. We establish the converse statement. We prove directly that these forms are nondegenerate and show how this nondegeneration implies the Decomposition Theorem. In the critical case of cohomological degree $n$ and perversity zero, we show that the graded class map $H_{n, 0}\left(f^{-1}(y)\right) \rightarrow H_{0}^{n}(X)$ is an injection of pure Hodge structures and that the refined intersection form on the fiber $f^{-1}(y)$ underlies a polarization. Again, our results can be seen as a "Decomposition Theorem with signs."

These results, coupled with a series of simple reductions, give a proof of (4), (5) for proper maps of complex algebraic varieties and of (3) for projective maps of complex algebraic varieties (cf. 2.3).

The Purity Theorem 2.2.1 states that the direct sum decomposition for the hypercohomology of (5) is by Hodge sub-structures. In particular, the intersection cohomology groups of projective varieties carry a pure Hodge structure which is canonical in the sense of 2.2.3(a).

The Hodge-Lefschetz Theorem for Intersection Cohomology 2.2.3 is a generalization to the intersection cohomology of a projective variety of the classical Hodge theory for the singular cohomology of projective manifolds (cf. 3.1).

What follows compares the results of the present paper with some of the literature.

Theorem 2.3.3 is proved by Beilinson, Bernstein, Deligne and Gabber in [1]. The result is first proved in positive characteristic using the formalism of perverse sheaves in conjunction with the purity results proved in [13] concerning the eigenvalues of the Frobenius operator acting on complexes of sheaves on a variety defined over a finite field. The result is then "lifted" to characteristic zero.

The deep elegance of this approach does not seem to explain the geometry of the result over the complex numbers and does not give a proof of the Hodge-theoretic results in 2.1. In the series of remarkable papers [26,27] and [28], M. Saito has developed a D-modules transcendental approach via his own mixed Hodge modules.

C. Sabbah [25] has recently extended M. Saito's results to the case of semisimple local systems on $X$ by developing his own theory of polarizable twistor D-modules. See also the related work by T. Mochizuki $[23,24]$.

While Saito's results cover and pre-date some of the results of this paper, namely the Relative Hard Lefschetz Theorem 2.1.1(a), the Decomposition Theorem 2.1.1(b) and part of the Purity of Intersection Cohomology 2.2.3(a), and give other results as well, the proofs do not seem to explain the underlying geometry and do not describe explicitly Hodge structures and polarizations.

We show that the properties of the refined intersection form are responsible for the topological splitting of $R f_{*} \mathbb{Q}_{X}[n]$ and we establish these properties using Hodge theory.

The paper is not self-contained as it relies, for instance, on the theory of $t$-structures. However, at several stages, we need results in a form that seems to be less general but sharper than what we could find in the literature. For this reason, we offer two rather long sections of preliminaries. We also hope that having collected results on the theory of stratifications, constructible sheaves and perverse sheaves can in any case be useful to the reader. The statements proved in this paper are collected in Section 2. The proofs of the main results are strongly intertwined and we give 
a detailed account of the steps of the proof in 2.6, trying to emphasize the main ideas. Due to the presence of a rich array of structures, many verifications of compatibility are necessary in the course of our proofs. We have decided to include careful proofs of the ones that did not seem to be just routine.

\section{Statements}

We state and prove our results for maps of projective varieties $f: X \rightarrow Y$ with $X$ nonsingular in 2.1 and 2.2. The Hodge-theoretic results are strongest and more meaningful in this context. Most results remain valid (cf. 2.3) for proper algebraic maps of algebraic varieties via standard reductions to the nonsingular projective case.

\subsection{The projective case}

The basic set-up of this paper is as follows:

- let $f: X \rightarrow Y$ be a map of projective varieties, $X$ nonsingular of dimension $n, \eta$ be an ample line bundle on $X, A$ be an ample line bundle on $Y$ and $L:=f^{*} A$.

The results that follow are discussed in the two Examples 2.4 and 2.5.

We denote $R f_{*}$ simply by $f_{*}$. The line bundle $\eta$ defines a map $\mathbb{Q}_{X} \rightarrow \mathbb{Q}_{X}[2]$. By pushingforward in the derived sense, we get a map $f_{*} \mathbb{Q}_{X} \rightarrow f_{*} \mathbb{Q}_{X}[2]$ and maps ${ }^{\eta} \mathcal{H}^{i}\left(f_{*} \mathbb{Q}_{X}[n]\right) \rightarrow$ ${ }^{p} \mathcal{H}^{i+2}\left(f_{*} \mathbb{Q}_{X}[n]\right)$. We denote all these maps simply by $\eta$.

The following extends a great deal of classical Hodge Theory to the case of maps. It was proved by Beilinson, Bernstein and Deligne in [1].

THEOREM 2.1.1. -

(a) (The Relative Hard Lefschetz Theorem). For every $i \geqslant 0$, the map induced by $\eta$ in perverse cohomology is an isomorphism:

$$
\eta^{i}:{ }^{p} \mathcal{H}^{-i}\left(f_{*} \mathbb{Q}_{X}[n]\right) \simeq{ }^{p} \mathcal{H}^{i}\left(f_{*} \mathbb{Q}_{X}[n]\right) .
$$

In particular, having set, for $i \geqslant 0, \mathcal{P}_{\eta}^{-i}:=\operatorname{Ker} \eta^{i+1} \subseteq{ }^{\mathcal{H}^{-i}}\left(f_{*} \mathbb{Q}_{X}[n]\right)$, we have equalities

$$
{ }^{p} \mathcal{H}^{-i}\left(f_{*} \mathbb{Q}_{X}[n]\right)=\bigoplus_{j \geqslant 0} \eta^{j} \mathcal{P}_{\eta}^{-i-2 j}, \quad{ }^{p} \mathcal{H}^{i}\left(f_{*} \mathbb{Q}_{X}[n]\right)=\bigoplus_{j \geqslant 0} \eta^{i+j} \mathcal{P}_{\eta}^{-i-2 j}
$$

(b) (The Decomposition Theorem). There is an isomorphism in $D(Y)$ :

$$
\varphi: \bigoplus_{i}^{p} \mathcal{H}^{i}\left(f_{*} \mathbb{Q}_{X}[n]\right)[-i] \simeq f_{*} \mathbb{Q}_{X}[n]
$$

(c) (The Semisimplicity Theorem). The ${ }^{p} \mathcal{H}^{i}\left(f_{*} \mathbb{Q}_{X}[n]\right)$ are semisimple (cf. 3.8). More precisely, given any stratification for $f$ (cf. 3.2) $Y=\amalg_{l} S_{l}, 0 \leqslant l \leqslant \operatorname{dim} Y$, there is a canonical isomorphism in $\operatorname{Perv}(Y)$ :

$$
{ }^{p} \mathcal{H}^{i}\left(f_{*} \mathbb{Q}_{X}[n]\right) \simeq \bigoplus_{l=0}^{\operatorname{dim} Y} I C_{\overline{S_{l}}}\left(L_{i, l}\right)
$$

where the local systems $L_{i, l}:=\alpha_{l}^{*} \mathcal{H}^{-l}\left({ }^{p} \mathcal{H}^{i}\left(f_{*} \mathbb{Q}_{X}[n]\right)\right)$ on $S_{l}$ are semisimple.

$4^{\mathrm{e}}$ SÉRIE - TOME $38-2005-\mathrm{N}^{\circ} 5$ 
Remark 2.1.2. - The complexes ${ }^{p} \mathcal{H}^{i}\left(f_{*} \mathbb{Q}_{X}[n]\right)=0$ if $|i|>r(f)$, where $r(f)$ is the defect of semismallness 4.7.2 of $f$. It can be shown that this vanishing is sharp (cf. [8]).

Remark 2.1.3. - The isomorphism $\varphi$ of Theorem 2.1.1(b) is not unique. It is possible to make some distinguished choices (cf. [10]). These choices play no role in the present paper.

The symbol $\stackrel{\varphi}{\simeq}$ indicates that a certain isomorphism is realized via $\varphi$. The Decomposition Theorem implies that (cf. 4.3), setting

$$
H_{\leqslant b}^{n+l}(X):=\operatorname{Im}\left\{\mathbb{H}^{l}\left(Y,{ }^{p} \tau_{\leqslant b} f_{*} \mathbb{Q}_{X}[n]\right) \rightarrow \mathbb{H}^{l}\left(Y, f_{*} \mathbb{Q}_{X}[n]\right)\right\} \subseteq H^{n+l}(X)
$$

and

$$
H_{b}^{n+l}(X):=H_{\leqslant b}^{n+l}(X) / H_{\leqslant b-1}^{n+l}(X),
$$

we get a canonical identification

$$
H_{b}^{n+l}(X)=\mathbb{H}^{l-b}\left(Y,{ }^{p} \mathcal{H}^{b}\left(f_{*} \mathbb{Q}_{X}[n]\right)\right)
$$

and isomorphisms:

$$
H_{\leqslant b}^{n+l}(X) \stackrel{\varphi}{\simeq} \bigoplus_{i \leqslant b} \mathbb{H}^{l-i}\left(Y,{ }^{p} \mathcal{H}^{i}\left(f_{*} \mathbb{Q}_{X}[n]\right)\right) .
$$

The cup product with $\eta$ verifies $\eta H_{\leqslant a}^{l}(X) \subseteq H_{\leqslant a+2}^{l+2}(X)$ (cf. 4.4) and induces maps, still denoted $\eta: H_{a}^{l}(X) \rightarrow H_{a+2}^{l+2}(X)$. The cup product with $L$ is compatible with the direct sum decomposition induced by any isomorphism $\varphi$ (cf. 4.4.3) and induces maps $L: H_{a}^{l}(X) \rightarrow$ $H_{a}^{l+2}(X)$.

Theorem 2.1.4 (The Hard Lefschetz Theorem for Perverse Cohomology Groups). - Let $k \geqslant 0, b, j \in \mathbb{Z}$. Then the following cup product maps are isomorphisms:

$$
\eta^{k}: H_{-k}^{j}(X) \simeq H_{k}^{j+2 k}(X), \quad L^{k}: H_{b}^{n+b-k}(X) \simeq H_{b}^{n+b+k}(X) .
$$

The previous result allows to describe the perverse filtration purely in terms of the nilpotent linear map $L$ acting via cup-product on the cohomology of $X$. For the precise statement, involving the notion of weight filtration associated with $L$, see 4.5, 4.6 and Proposition 5.2.4. Since $L$ is of type $(1,1)$, we get the following.

THEOREM 2.1.5 (The Hodge Structure Theorem). - For $l \geqslant 0$ and $b \in \mathbb{Z}$, the subspaces

$$
H_{\leqslant b}^{l}(X) \subseteq H^{l}(X)
$$

are pure Hodge sub-structures. The quotient spaces

$$
H_{b}^{l}(X)=H_{\leqslant b}^{l}(X) / H_{\leqslant b-1}^{l}(X)
$$

inherit a pure Hodge structure of weight $l$.

Note that the Hodge structure thus constructed on $H_{b}^{l}(X)$ is compatible with restriction to zero-loci of sections of $\eta$ and $L$ in the following sense. Let $i: X_{s}^{r} \rightarrow X$ be the inclusion of a complete intersection of $r$ general sections of $\eta$ and $s$ general sections of $L$. The restriction $i^{*}: H^{l}(X) \rightarrow H^{l}\left(X_{s}^{r}\right)$ maps $H_{\leqslant a}^{l}(X)$ to $H_{\leqslant a+r}^{l}\left(X_{s}^{r}\right)$ (cf. 4.2.3) and induces a morphism of pure Hodge structures $H_{a}^{l}(X) \rightarrow H_{a+r}^{l}\left(X_{s}^{r}\right)$. 
Remark 2.1.6. - The isomorphism $\varphi$ may not be compatible with the Hodge structure, that is the isomorphisms

$$
H^{l}(X) \stackrel{\varphi}{\simeq} \bigoplus_{b} H_{b}^{l}(X)
$$

are not necessarily of Hodge structures. This, though, can be achieved with a particular choice of $\varphi$.

Define $P_{-i}^{-j}:=\operatorname{Ker} \eta^{i+1} \cap \operatorname{Ker} L^{j+1} \subseteq H_{-i}^{n-i-j}(X), i, j \geqslant 0$ and $P_{-i}^{-j}:=0$ otherwise. In the same way in which the classical Hard Lefschetz implies the Primitive Lefschetz Decomposition for the cohomology of $X$, Theorem 2.1.4 implies the double direct sum decomposition of

COROLlaRY 2.1.7 (The ( $\eta, L)$-Decomposition). - Let $i, j \in \mathbb{Z}$. There is a Lefschetz-type direct sum decomposition into pure Hodge sub-structures of weight $(n-i-j)$, called the $(\eta, L)$ decomposition:

$$
H_{-i}^{n-i-j}(X)=\bigoplus_{l, m \in \mathbb{Z}} \eta^{-i+l} L^{-j+m} P_{i-2 l}^{j-2 m}
$$

Using representatives in $H_{\leqslant-i}^{n-i-j}(X), i, j \geqslant 0$, define bilinear forms $S_{i j}^{\eta L}$ on $H_{-j}^{n-i-j}(X)$ by modifying the Poincaré pairing

$$
S_{i j}^{\eta L}([\alpha],[\beta]):=\int_{X} \eta^{i} \wedge L^{j} \wedge \alpha \wedge \beta .
$$

Using 2.1.4, one can define forms $S_{i j}^{\eta L}$ for all $i$ and $j$. These forms are well defined and nondegenerate (cf. 4.5).

TheOREM 2.1.8 (The Generalized Hodge-Riemann Bilinear Relations). - The ( $\eta, L)$-decomposition is orthogonal with respect to $S_{i j}^{\eta L}$. The forms $S_{i j}^{\eta L}$ are, up to a sign made precise in 4.5.2, a polarization of each $(\eta, L)$-direct summand.

The following two results are key in proving the Semisimplicity Theorem and in our opinion are geometrically significant. For what follows see 3.4 and Lemma 4.3.6.

The homology groups $H_{*}^{\mathrm{BM}}\left(f^{-1}(y)\right)=H_{*}\left(f^{-1}(y)\right), y \in Y$, are endowed with the perverse filtration, see 4.3.5. The natural cycle class map $c l: H_{n-*}^{\mathrm{BM}}\left(f^{-1}(y)\right) \rightarrow H^{n+*}(X)$ is strict.

THEOREM 2.1.9 (The Generalized Grauert Contractibility Criterion). - Let $b \in \mathbb{Z}, y \in Y$. The natural class maps

$$
c l_{b}: H_{n-b, b}^{\mathrm{BM}}\left(f^{-1}(y)\right) \rightarrow H_{b}^{n+b}(X)
$$

is injective and identifies $H_{n-b, b}^{\mathrm{BM}}\left(f^{-1}(y)\right) \subseteq \operatorname{Ker} L \subseteq H_{b}^{n+b}(X)$ with a pure Hodge substructure, compatibly with the $(\eta, L)$-decomposition. Each $(\eta, L)$-direct summand of $H_{n-b, b}^{\mathrm{BM}}\left(f^{-1}(y)\right)$ is polarized up to sign by $S_{-b, 0}^{\eta L}$.

In particular, the restriction of $S_{-b, 0}^{\eta L}$ to $H_{n-b, b}^{\mathrm{BM}}\left(f^{-1}(y)\right)$ is nondegenerate.

By intersecting in $X$ cycles supported on $f^{-1}(y)$, we get the refined intersection form (cf. 3.4) $H_{n-*}^{\mathrm{BM}}\left(f^{-1}(y)\right) \rightarrow H^{n+*}\left(f^{-1}(y)\right)$ which is strict as well.

THEOREM 2.1.10 (The Refined Intersection Form Theorem). - Let $b \in \mathbb{Z}, y \in Y$. The graded refined intersection form

$$
H_{n-b, a}^{\mathrm{BM}}\left(f^{-1}(y)\right) \rightarrow H_{a}^{n+b}\left(f^{-1}(y)\right)
$$

is zero if $a \neq b$ and it is an isomorphism if $a=b$.

$4^{\text {e }}$ SÉRIE - TOME $38-2005-\mathrm{N}^{\circ} 5$ 
Remark 2.1.11. - Let $b \in \mathbb{Z}, y \in Y$. If $y$ lies on a positive-dimensional stratum $S_{l}$ for the map $f$ (cf. 3.2), then the refined intersection form is identically zero (cf. 3.4.1). In this case, the geometrically interesting map is the one of the Splitting Criterion 4.1.3: $H_{n-l-b, b}^{\mathrm{BM}}\left(f^{-1}(y)\right) \rightarrow$ $H_{b}^{n-l+b}\left(f^{-1}(y)\right)$. This map is the refined intersection form for $f^{-1}(y)$ in $X_{l}$ for the map $X_{l} \rightarrow Y_{l}$, where $Y_{l}$ is a codimension- $l$ general complete intersection on $Y$ transversal to $S_{l}$ at $y$ and $X_{l}:=f^{-1}\left(Y_{l}\right)$ (cf. 4.3.8).

The following two corollaries give examples of the kind of geometric applications stemming from the results of this paper. See also Example 2.4.

Corollary 2.1.12 (Contractibility Criterion). - Let $f: X \rightarrow Y$ be a projective and surjective map of quasi-projective varieties, $X$ nonsingular. Let $y \in Y$ and assume that $f$ is semismall over $Y \backslash y$, e.g. $f_{\mid X \backslash f^{-1}(y)}$ is an isomorphism.

For every $b \geqslant 0$ the natural mixed Hodge structure $H^{n+b}\left(f^{-1}(y)\right)$ is pure of weight $n+b$. The associated $\eta$-direct summands are polarized up to sign by the forms $S_{-b 0}^{\eta L}$.

Proof. - We may compactify the morphism without changing the situation around and over $y$. In particular, the perverse cohomology complexes will not change around $y$ by this process. It follows that we may assume that $X$ is projective. By the Decomposition Theorem, the Semisimplicity Theorem and semismallness (cf. 4.7.2 and 4.7.3)

$$
f_{*} \mathbb{Q}_{X}[n] \simeq \bigoplus_{l>0} I C_{\bar{S}_{l}}\left(L_{0, l}\right) \oplus T^{0} \oplus \bigoplus_{i \neq 0}{ }^{p} \mathcal{H}^{i}\left(f_{*} \mathbb{Q}_{X}[n]\right)[-i],
$$

where $T^{0}$ and ${ }^{p} \mathcal{H}^{i}\left(f_{*} \mathbb{Q}_{X}[n]\right)$ are skyscraper sheaves at $y$. It follows that the natural map $H^{n+b}(X) \rightarrow H^{n+b}\left(f^{-1}(y)\right)$ is surjective for every $b \geqslant 0$, whence the purity statement. Since $\mathcal{H}^{j}\left(I C_{\bar{S}_{l}}\left(L_{0, l}\right)\right)=0$ for $j \geqslant 0$ (cf. 3.8), $H^{n}\left(f^{-1}(y)\right)=T^{0}$ and $H^{n+b}\left(f^{-1}(y)\right)=$ $p \mathcal{H}^{b}\left(f_{*} \mathbb{Q}_{X}[n]\right)$ for $b>0$. It follows that, for $b \geqslant 0$, we have $H^{n+b}\left(f^{-1}(y)\right)=H_{\leqslant b}^{n+b}\left(f^{-1}(y)\right)=$ $H_{b}^{n+b}\left(f^{-1}(y)\right)$. The result follows from 2.1.10 and 2.1.9.

Remark 2.1.13. - If, for example, $n=4$ and $f^{-1}(y)=\bigcup S_{j}$ is a configuration of surfaces, then the matrix $\left[S_{j}\right] \cdot\left[S_{k}\right]$ is positive definite. The example of $\mathbb{P}^{2} \subseteq \mathbb{P}^{4}$ shows that the necessary Contractibility conditions expressed by Corollary 2.1 .12 are clearly not sufficient.

In [6] we study projective semismall (cf. 4.7.2) maps $f: X \rightarrow Y$ with $X$ a projective manifold. We show that the intersection form $I$ associated with a component of a codimension $2 h$ relevant stratum has a precise signature: $(-1)^{h} I>0$. The methods of [6] do not cover the case of semismall projective maps from a quasi-projective manifold $X$. One may compactify the morphism, but the condition of semismallness could be destroyed at the boundary. The methods of this paper by-pass this problem and we have the following

COROLlary 2.1.14 (Signature Theorem for Semismall Maps). - Let $f: X \rightarrow Y$ be a projective and surjective semismall map, with $X$ nonsingular and quasi-projective. Then the intersection form $I$ associated with a component of a codimension $2 h$ relevant stratum is $(-1)^{h} I>0$.

Proof. - As in the proof of 2.1.12, we may also assume that $X$ is projective. By slicing with hyperplane sections on $Y$ (cf. Section 6 and [6]), we are reduced to the case when $\operatorname{dim} X=h$, i.e. to the zero-dimensional stratum $S_{0}$. Let $y \in S_{0}$. We have a decomposition similar to the one in the proof of 2.1.12, where $T^{0} \simeq H_{n}\left(f^{-1}(y)\right)$ is polarized up to sign by the intersection form on $X$, i.e. by $I$. 


\subsection{Purity and Hodge-Lefschetz for intersection cohomology}

Let $H_{i, l}^{j}(X):=\mathbb{H}^{j-i-n}\left(Y, I C_{\bar{S}_{l}}\left(L_{i, l}\right)\right) \subseteq H_{i}^{j}(X), 0 \leqslant l \leqslant \operatorname{dim} Y, i \in \mathbb{Z}, j \geqslant 0$. See 2.1.1(c). In general, the pure-dimensional stratum $S_{l}$ is not connected and one may write

$$
H_{i, l}^{j}(X)=\bigoplus_{S} H_{i, l, S}^{j}(X)
$$

where the direct sum is over the connected components $S$ of $S_{l}$.

A priori, it is not clear that the 1.h.s. of (6) is a Hodge sub-structure of $H_{i}^{j}(X)$ and that (6) itself is a decomposition into Hodge sub-structures. We prove these facts in the following

THEOREM 2.2.1 (Purity Theorem). - Let $j \geqslant 0$ and $i \in \mathbb{Z}$. The canonical direct sum decomposition

$$
H_{i}^{j}(X)=\bigoplus_{l} H_{i, l}^{j}(X)=\bigoplus_{l, S} H_{i, l, S}^{j}(X)
$$

is by Hodge sub-structures, it is $S^{\eta L}$-orthogonal, and it is compatible with the $(\eta, L)$-decomposition and its polarization.

Remark 2.2.2. - The spaces $H_{b, l, S}^{n+b+d}(X)$ behave like the cohomology of a collection of projective manifolds. Fix $b \in \mathbb{Z}, l \geqslant 0$ and $S$ a connected component of $S_{l}$, and let $d \in \mathbb{Z}$ vary. We get a structure which satisfies Hodge-Lefschetz properties for $L$ analogous to the ones stated in Theorem 2.2.3. Similarly, if we fix $d, l$ and $S$, and we let $b \in \mathbb{Z}$ vary, then we get similar properties with respect to $\eta$. See 2.4, 2.5.

The following is the intersection cohomology counterpart of the classical Hodge Theory of projective manifolds.

THEOREM 2.2.3 (Hodge-Lefschetz for Intersection Cohomology). -

(a) (Purity of Intersection Cohomology). For every $j \geqslant 0$, the intersection cohomology group $I H^{j}(Y, \mathbb{Q})$ carries a weight- $j$ pure Hodge structure.

This structure is characterized by the property that, given any projective resolution $f: X \rightarrow Y$, the resulting natural inclusion

$$
I H^{j}(Y, \mathbb{Q}) \rightarrow H_{0}^{j}(X)
$$

is a map of weight- $j$ pure Hodge structures.

Moreover, given a diagram of projective resolutions

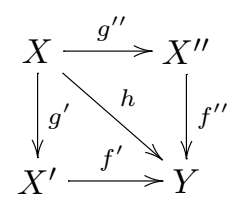

the images of the natural inclusions

$$
I H^{j}(Y, \mathbb{Q}) \stackrel{f^{\prime} \circ g^{\prime}}{\longrightarrow} H_{0}^{j}(X) \stackrel{f^{\prime \prime} \circ g^{\prime \prime}}{\longleftarrow} I H^{j}(Y, \mathbb{Q})
$$

coincide.

(b) (Weak Lefschetz Theorem for Intersection Cohomology). Let $r: Y_{1} \subseteq Y$ be the inclusion of a subvariety hyperplane section of $Y$ transversal to all the strata of a stratification

$4^{\mathrm{e}}$ SÉRIE - TOME $38-2005-\mathrm{N}^{\circ} 5$ 
of $Y$. Then

$$
r^{*}: I H^{j}(Y, \mathbb{Q}) \rightarrow I H^{j}\left(Y_{1}, \mathbb{Q}\right)
$$

is an isomorphism for $j \leqslant \operatorname{dim} Y-2$ and injective for $j=\operatorname{dim} Y-1$.

(c) (Hard Lefschetz Theorem for Intersection Cohomology) The cup product map

$$
A^{j}: I H^{\operatorname{dim} Y-j}(Y, \mathbb{Q}) \rightarrow I H^{\operatorname{dim} Y+j}(Y, \mathbb{Q})
$$

is an isomorphism for every $j \geqslant 0$. Setting $P_{A}^{n-r}:=\operatorname{Ker} A^{r+1} \subseteq I H^{n-r}(Y, \mathbb{Q}), r \geqslant 0$ there is a primitive Lefschetz decomposition

$$
I H^{\operatorname{dim} Y-j}(Y, \mathbb{Q})=\bigoplus_{r \geqslant 0} A^{r} P_{A}^{\operatorname{dim} Y-j-2 r} .
$$

(d) (Hodge-Riemann Bilinear Relations for Intersection Cohomology) Given a projective resolution $f: X \rightarrow Y$ of the singularities of $Y$, the forms $S_{0,-j}^{\eta L}$ polarize, up to sign, the spaces $P_{A}^{n-j}$. Moreover, the decomposition (8) is $S_{0,-j}^{\eta L}$-orthogonal.

Remark 2.2.4. - The Hodge-Lefschetz Theorem 2.2.3 is due to several authors. The Weak Lefschetz Theorem is due to Goresky and MacPherson [17]. The Hard Lefschetz Theorem for Intersection Cohomology is proved in [1] using algebraic geometry in positive characteristic. We prove it as a corollary to the Hard Lefschetz Theorem on Perverse Cohomology Groups. The fact that the Intersection Cohomology of a projective variety carries a canonical pure Hodge structure is proved by M. Saito in [26]. The relation with the cohomology of resolutions is stated, but not proved in [28]. Polarizations associated with Intersection Cohomology appear implicitly in Saito's work. The methods of the aforementioned papers are completely different from the ones of this paper.

\subsection{The algebraic case}

In this section we point out that a series of simple reductions using Hironaka's resolution of singularities and Chow's Lemma allows to prove Theorem 2.1.1 for proper maps of algebraic varieties. Since no new idea is necessary for this purpose, we omit the proofs. For details see [8], p. 71-74.

THEOREM 2.3.1. - Let $f: X \rightarrow Y$ be a proper map of projective varieties. Then all the results in 2.1 hold if we replace $\mathbb{Q}_{X}[n]$ with $I C_{X}$.

Remark 2.3.2. - In the singular case above, the refined intersection form must be replaced by the maps $\mathbb{H}^{*}\left(y, \alpha_{y}^{!} f_{*} I C_{X}\right) \rightarrow \mathbb{H}^{*}\left(y, \alpha_{y}^{*} f_{*} I C_{X}\right)$, where $\alpha_{y}: y \rightarrow Y$ is a point in $Y$.

TheOREM 2.3.3. - Let $f: X \rightarrow Y$ be a proper map of algebraic varieties. Then the Decomposition Theorem 2.1.1(b) and the Semisimplicity Theorem 2.1.1(c) hold for $f$ if we replace $\mathbb{Q}_{X}[n]$ by $I C_{X}$.

If, in addition, $f$ is projective and $\eta$ is $f$-ample, then the Relative Hard-Lefschetz Theorem 2.1.1(a) holds as well.

The results hold, with obvious modifications also left to the reader, for the push-forward of any complex $K \simeq \bigoplus_{i}{ }^{p} \mathcal{H}^{i}(K)[-i]$ such that each ${ }^{p} \mathcal{H}^{i}(K) \simeq \bigoplus I C_{\bar{Z}}\left(L_{Z}\right)$, where the $Z$ are nonsingular locally closed subvarieties of $X$ and the $L_{Z}$ are self-dual local systems arising as direct summands of some ${ }^{p} \mathcal{H}^{b}\left(g_{*} \mathbb{Q}_{Z^{\prime}}\left[\operatorname{dim} Z^{\prime}\right]\right)$ for some algebraic proper $g: Z^{\prime} \rightarrow Z$. 


\subsection{Example: Resolution of singularities of a threefold}

Let $f: X \rightarrow Y$ be a generically finite and surjective map from a nonsingular three-dimensional projective variety $X$. For ease of exposition, we assume that $f$ admits a stratification $Y=$ $U \amalg C \amalg y$ where $y$ is a point in the closure of the smooth curve $C$ with the properties: (a) $\operatorname{dim} f^{-1}(y)=2$, (b) $\operatorname{dim} f^{-1}(c)=1, c \in C$. The map $f$ is locally topologically trivial, when restricted to the strata.

Note that $f$ is semismall over $Y \backslash y$. Let $\mathcal{L}_{U}$ be the local system on $U$ associated with the topological covering $f^{-1}(U) \rightarrow U$ and $\mathcal{L}_{C}$ be the local system on $C$ associated with $H^{2}\left(f^{-1}(c)\right), c \in C$. Let $D:=f^{-1}(y), D_{j} \subseteq D$ be the irreducible surface components. We have that $H_{4}(D)$ is spanned precisely by the fundamental classes of $\left\{D_{j}\right\}$. Denote by $\left[D_{j}\right] \in H^{2}(X)$ the image of $\left\{D_{j}\right\}$ via the natural class map $c l: H_{4}(D) \rightarrow H^{2}(X)$. Let $X^{1} \subseteq X$ be a general $\eta$-hyperplane section of $X$ and $X_{1}$ be the zero locus of a general section of $L=f^{*} A$. The map $f_{\mid}: X^{1} \rightarrow Y$ is generically finite onto a surface and contracts to $y$ the irreducible curves $E_{j}:=D_{j} \cap X^{1}$. The map $f_{\mid}: X_{1} \rightarrow Y$ has analogous properties, but contracts the irreducible curves inside $f^{-1}(c), c \in C \cap f\left(X_{1}\right)$.

The defect of semismallness $r(f)=1$ so that ${ }^{p} \mathcal{H}^{i}\left(f_{*} \mathbb{Q}_{X}[3]\right)=0, i \neq-1,0,1$ and

\begin{tabular}{|l|l|l|}
\hline${ }^{p} \mathcal{H}^{-1}\left(f_{*} \mathbb{Q}_{X}[3]\right)$ & ${ }^{p} \mathcal{H}^{0}\left(f_{*} \mathbb{Q}_{X}[3]\right)$ & ${ }^{p} \mathcal{H}^{1}\left(f_{*} \mathbb{Q}_{X}[3]\right)$ \\
\hline$H_{4}(D)_{y}$ & $I C_{Y}\left(\mathcal{L}_{U}\right) \oplus I C_{\bar{C}}\left(\mathcal{L}_{C}\right) \oplus H_{3}(D)_{y}$ & $H^{4}(D)_{y}$ \\
\hline
\end{tabular}

If $f^{-1}(c)$ is irreducible, then $I C_{\bar{C}}\left(\mathcal{L}_{C}\right) \simeq \nu_{*} \mathbb{Q}_{\widetilde{C}}[1]$, where $\nu: \widetilde{C} \rightarrow \bar{C}$ is the normalization.

The Relative Hard Lefschetz Theorem, i.e. $\eta^{p}{ }^{p} \mathcal{H}^{-1}\left(f_{*} \mathbb{Q}_{X}[3]\right) \simeq{ }^{p} \mathcal{H}^{1}\left(f_{*} \mathbb{Q}_{X}[3]\right)$, becomes the statement that the composition $H_{4}(D) \rightarrow H^{2}(X) \stackrel{\eta}{\rightarrow} H^{4}(X) \rightarrow H^{4}(D)$ is an isomorphism so that the ensuing bilinear form on $H_{4}(D)$

$$
\left\langle\left\{D_{j}\right\},\left\{D_{k}\right\}\right\rangle=\int_{X} \eta \wedge\left[D_{j}\right] \wedge\left[D_{k}\right]=\int_{X^{1}}\left[E_{j}\right] \wedge\left[E_{k}\right]
$$

is nondegenerate. We have ${ }^{\mathcal{P}} \mathcal{H}^{-i}\left(f_{*} \mathbb{Q}_{X}[3]\right)=\mathcal{P}_{\eta}^{-i}, i=0,1$.

The Decomposition Theorem reads:

$$
f_{*} \mathbb{Q}_{X}[3] \simeq\left(H_{4}(D)_{y}[1]\right) \oplus\left(I C_{Y}\left(\mathcal{L}_{U}\right) \oplus I C_{\bar{C}}\left(\mathcal{L}_{C}\right) \oplus H_{3}(D)_{y}[0]\right) \oplus\left(H^{4}(D)_{y}[-1]\right) .
$$

The Semisimplicity Theorem implies the elementary fact that $\mathcal{L}_{U}$ and $\mathcal{L}_{C}$ are semisimple.

The Hodge Structure Theorem gives Table 1 of Hodge (sub-)structures. The forms $S_{i j}^{\eta L}$ are defined via $\int_{X} \eta^{i} \wedge L^{j} \wedge a \wedge b=\int_{X_{j}^{i}} a_{\mid X_{j}^{i}} \wedge b_{\mid X_{j}^{i}}$.

Table 1

\begin{tabular}{llllllll}
\hline coh.deg. & 0 & 1 & 2 & 3 & 4 & 5 & 6 \\
\hline$H_{\leqslant-1}^{*}(X)$ & 0 & 0 & $\operatorname{Im}\left\{H_{4}(D) \rightarrow H^{2}\right\}$ & 0 & 0 & 0 & 0 \\
$H_{\leqslant 0}^{*}(X)$ & $H^{0}$ & $H^{1}$ & $H^{2}$ & $H^{3}$ & $\operatorname{Ker}\left\{H^{4} \rightarrow H^{4}(D)\right\}$ & $H^{5}$ & $H^{6}$ \\
$H_{\leqslant 1}^{*}(X)$ & $H^{0}$ & $H^{1}$ & $H^{2}$ & $H^{3}$ & $H^{4}$ & $H^{5}$ & $H^{6}$ \\
\hline
\end{tabular}

$4^{\mathrm{e}}$ SÉRIE - TOME $38-2005-\mathrm{N}^{\circ} 5$ 
Table 2

\begin{tabular}{llllllll}
\hline coh.deg. & 0 & 1 & 2 & 3 & 4 & 5 & 6 \\
\hline$H_{-1}^{*}(X)$ & 0 & 0 & $P_{-1}^{0} \simeq H_{4}(D)$ & 0 & 0 & 0 & 0 \\
$H_{0}^{*}(X)$ & $P_{0}^{-3}$ & $P_{0}^{-2}$ & $P_{0}^{-1} \oplus L P_{0}^{-3}$ & $P_{0}^{0} \oplus L P_{0}^{-2}$ & $L P_{0}^{-1} \oplus L^{2} P_{0}^{-3}$ & $L^{2} P_{0}^{-2}$ & $L^{3} P_{0}^{-3}$ \\
$H_{1}^{*}(X)$ & 0 & 0 & 0 & 0 & $\eta P_{-1}^{0} \simeq H^{4}(D)$ & 0 & 0 \\
\hline
\end{tabular}

The $(\eta, L)$-Decomposition Theorem and the Generalized Hodge-Riemann Bilinear Relations give Table 2 of $S_{i j}^{\eta L}$-orthogonal decompositions of the pure Hodge structures $H_{-i}^{3-i-j}(X)$. Each term $P_{-i}^{-j}$ is a Hodge sub-structure polarized by $S_{i j}^{\eta L}$. In general, the display above has several rows. Each row presents a horizontal shifted symmetry with respect to the action of $L$. There is an additional diagonal symmetry of the display due to $\eta$.

Each horizontal and diagonal row behaves like the cohomology of a projective manifold under the action of an ample line bundle.

These symmetries are explained by the Hard Lefschetz Theorem for Perverse Cohomology Groups which gives the following display of isomorphisms

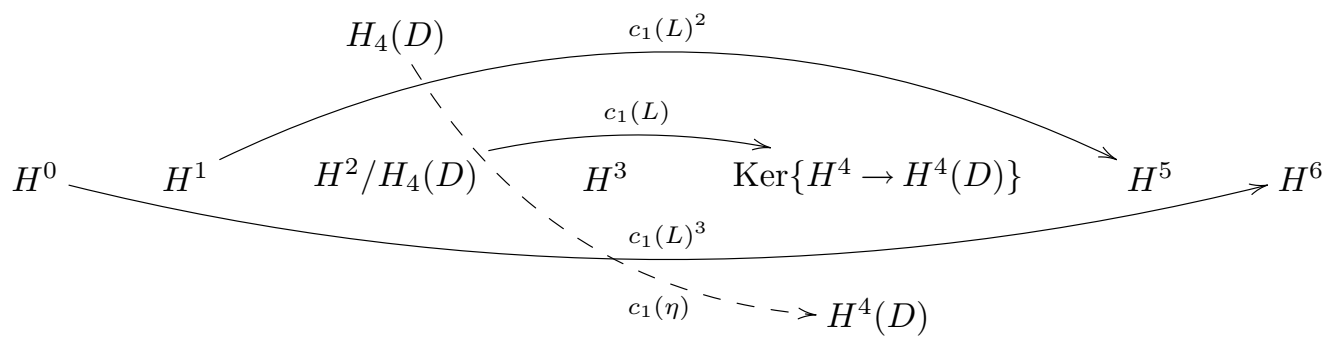

The Purity Theorem implies that the various pieces decompose further according to strata into polarized Hodge sub-structures in a fashion compatible with the ( $\eta, L)$-decomposition (cf. 2.2.2).

By the Generalized Grauert Contractibility Criterion, the fibers of $f$ contribute to the pieces $P_{-1}^{0}, P_{0}^{-1}$ and $P_{0}^{0}$ as we now explain.

(a) The class map $H_{4,-1}(D)=H_{4}(D) \rightarrow H_{-1}^{2}(X)$ is injective. The image is the Hodge structure $P_{-1}^{0}$ polarized by $S_{10}^{\eta L}$ which in turn coincides with the negative-definite (9). This is Grauert's Criterion for the contraction of the curves $E_{j}$ on $X^{1}$.

(b) The group $H_{2,1}\left(f^{-1}(c)\right)=\{0\}$ and the injectivity statement 2.1.9 is trivial for $c \in C$. The map $H_{2,0}\left(f^{-1}(c)\right)=H_{2}\left(f^{-1}(c)\right) \rightarrow H_{0}^{4}(X)$ lands in $L P_{0}^{-1}$. This map is not the zero map: its image is isomorphic to the invariants $H_{2}\left(f^{-1}(c)\right)^{\pi_{1}(C, c)}$. The fact that $S_{0,-1}^{\eta L}$ is a polarization merely reflects the Grauert criterion for the contraction of the curves in $f^{-1}(c), c \in C \cap f\left(X_{1}\right)$, given by the map $f_{\mid X_{1}}: X_{1} \rightarrow Y$.

(c) The class map $H_{3,0}(D)=H_{3}(D) \rightarrow H^{3}(X)$ is injective. The image is a split Hodge substructure of $P_{0}^{0}$. Both Hodge structures are polarized by $S_{00}^{\eta L}$, i.e. by $\int_{X}$. In fact, by the Contractibility Criterion 2.1.12: if $D$ is to appear in such a morphism $f$, then its a-priori mixed Hodge structures $H^{i}(D)$ must be pure for every $i \geqslant 3$.

\subsection{Example: Families of varieties}

Let $f: X \rightarrow C$ be a surjective map of projective manifolds, $\operatorname{dim} X=m+1, \operatorname{dim} C=1$.

There is a stratification of $f$ given by $C=U \amalg S$, where $S$ is a finite set. Denote by $g: f^{-1}(U)=: U^{\prime} \rightarrow U$ and by $\beta: U \rightarrow C \leftarrow S: \alpha$ the resulting maps. If $\mathcal{L}$ is a local system 
on $U$, then $I C_{C}(\mathcal{L})=\left(\beta_{*}^{0} \mathcal{L}\right)[1]$, where we denote by $\beta_{*}^{0}$ the sheaf-theoretic direct image (i.e. not the derived functor). We have $r(f)=m$ and

$$
\begin{aligned}
& p_{\mathcal{H}^{j}}\left(f_{*} \mathbb{Q}_{X}[m+1]\right) \simeq \beta_{*}^{0}\left(R^{m+j} g_{*} \mathbb{Q}_{U^{\prime}}\right)[1] \oplus K^{j}, \quad j \in[-m, m], \\
& 0 \quad \text { otherwise, }
\end{aligned}
$$

where the $K^{j}$ are sheaves supported on $S$.

The Relative Hard Lefschetz Theorem translates into

$$
\eta^{j}: R^{m-j} g_{*} \mathbb{Q}_{U^{\prime}} \simeq R^{m+j} g_{*} \mathbb{Q}_{U^{\prime}}, \quad \eta^{j}: K^{-j} \simeq K^{j} .
$$

The first isomorphism is the classical Hard Lefschetz Theorem for the fibers of the smooth map $g$.

The Decomposition Theorem reads

$$
f_{*} \mathbb{Q}_{X}[m+1] \simeq \bigoplus_{j}\left(\left(\beta_{*}^{0} R^{m+j} g_{*} \mathbb{Q}_{U^{\prime}}\right)[1][-j] \oplus \alpha_{*} K^{j}[-j]\right) .
$$

The Semisimplicity Theorem gives the well-known semisimplicity of the local systems $R^{m+j} g_{*} \mathbb{Q}_{U^{\prime}}$. We omit drawing tables as in 2.4. We point out that the stalks $K_{p}^{j}, p \in S$, are split Hodge sub-structures of $P_{-j}^{0}$, polarized up to sign by the forms $S_{j 0}^{\eta L}$. By taking cohomology sheaves we find isomorphisms $R^{i} f_{*} \mathbb{Q}_{X} \simeq \beta_{*}^{0} \beta^{*} R^{i} g_{*} \mathbb{Q}_{U^{\prime}} \oplus \alpha_{*} K^{i-m}$. It follows that the natural adjunction map $R^{i} f_{*} \mathbb{Q}_{X} \rightarrow \beta_{*}^{0} \beta^{*} R^{i} g_{*} \mathbb{Q}_{U^{\prime}}$ is surjective. By taking stalks at $p \in S$ we get that

$$
H^{i}\left(f^{-1}(p)\right) \rightarrow H^{i}\left(g^{-1}(u)\right)^{\mathbb{Z}}, \quad u \in U
$$

is surjective, i.e. that the classes in $H^{i}\left(g^{-1}(u)\right)$ which are invariant under the local monodromy around $p$ come from $H^{i}\left(f^{-1}(p)\right)$ or, equivalently, from $H^{i}\left(f^{-1}\left(U_{p}\right)\right), U_{p}$ a small Euclidean neighborhood of $p$. This statement is known as the Local Invariant Cycle Theorem. We note that, compared with the sharp versions of this theorem, due to various authors, see for instance $[30,5,15]$ and [18], this proof works only for projective (as opposed to Kähler) families over a quasi-projective base (as opposed to over the disk).

\subsection{The structure of the proof}

The set-up is as in 2.1. The proof of the results in 2.1 is by a double induction on the defect of semismallness and on the dimension of the target of the map $f: X \rightarrow Y$.

The Purity Theorem 2.2.1 is proved in 7.1. The Hodge-Lefschetz Theorem 2.2.3 is proved in 7.2 .

The starting point of the proof by induction is the following

Fact 2.6.1. - If $\operatorname{dim} f(X)=0$, then $L$ and the perverse filtration are trivial and all the results of 2.1 are either trivial or hold by classical Hodge theory. See Theorem 3.1.2.

The inductive hypothesis takes the following form

Assumption 2.6.2. - Let $R \geqslant 0$ and $m>0$. Assume that the results of 2.1 hold for every projective map $g: Z^{\prime} \rightarrow Z$ of projective varieties with $Z^{\prime}$ nonsingular such that either $r(g)<R$, or $\operatorname{dim} f(Z)<m$ and $r(g) \leqslant R$.

We prove that if Assumption 2.6.2 holds, then the results of 2.1 hold for every map $f: X \rightarrow Y$ as in 2.1 with $r(f) \leqslant R$ and $\operatorname{dim} f(X) \leqslant m$.

$4^{\mathrm{e}}$ SÉRIE - TOME $38-2005-\mathrm{N}^{\circ} 5$ 
In view of Fact 2.6.1, all the results in 2.1 follow by induction.

What follows is an outline of the structure of the proof of the results of this paper.

Remark 2.6.3. - We assume 2.6.2 and prove the results in 2.1 for the map $f$. Once a result has been established for $f$ we use it in the proof of the results that follow.

- Step 1. We prove the Relative Hard Lefschetz Theorem 2.1.1(a) in Proposition 5.1.3. Either the defect of semismallness is zero and there is nothing to prove, or we consider the universal hyperplane section morphism $g$ (cf. 4.7). In this case, the defect of semismallness $r(g)<$ $r(f)$ and the inductive hypothesis 2.6 .2 apply. The rest of the proof follows a classical path: one is reduced to the case $i=1$ and concludes by using the inductive semisimplicity statement for $g$. As is well-known (cf. [10]), the Decomposition Theorem 2.1.1(b) is a formal homological consequence of the Relative Hard Lefschetz Theorem 2.1.1(a). The Weak-Lefschetz-type result 4.7.8 implies the Semisimplicity Theorem 2.1.1(c) for $i \neq 0$. The critical case $i=0$ is proved at the end of the inductive procedure. See Step 6.

- Step 2. We prove the Hard Lefschetz Theorem for Perverse Cohomology Groups 2.1.4 in 5.2. The statement for $\eta$ follows from the Relative Hard Lefschetz after taking hypercohomology. Using hyperplane sections $X^{1}$ on $X$ (cf. 4.7.6) and $Y_{1}$ on $Y$ (cf. 4.7.7), the statement for $L$ is reduced to checking that $L: H_{0}^{n-1}(X) \simeq H_{0}^{n+1}(X)$. At this stage we do not know yet that $H_{0}^{n-1}(X)$ has a Hodge structure, so that care is needed when using Hodge-theoretic statements. We use the map $X_{1}=f^{-1}\left(Y_{1}\right) \rightarrow Y_{1}$ and the inductive Generalized Hodge-Riemann Bilinear Relations 2.1.8 to conclude.

- Step 3. The Hodge Structure Theorem 2.1.5 is proved in 5.2. The Hard Lefschetz Theorem for Perverse Cohomology Groups implies that the filtration $W^{\text {tot }}$ (cf. 4.6) on $\bigoplus_{j} H^{j}(X)$ coincides with the weight filtration $W^{L}$ for the nilpotent operator $L$. Since the latter is clearly Hodge-Theoretic, so is the former, whence Theorem 2.1.5. The $(\eta, L)$ Decomposition 2.1.7 is then a formal algebraic consequence of what has already been proved.

- Step 4. The Generalized Hodge-Riemann Bilinear Relations 2.1.8 are proved in the cases $P_{i}^{j} \neq P_{0}^{0}$ in 5.3 using the inductive hypothesis. The crucial case $P_{0}^{0}$ is proved in 5.4. Let $\varepsilon>0$ and $L_{\varepsilon}:=L+\varepsilon \eta: H^{n}(X) \rightarrow H^{n+2}(X), \Lambda_{\varepsilon}:=\operatorname{Ker} L_{\varepsilon} \subseteq H^{n}(X)$. We consider the pure Hodge structure $\Lambda=\lim _{\varepsilon \rightarrow 0} \Lambda_{\varepsilon} \subseteq \operatorname{Ker} L \subseteq H^{n}(X)$. Since, by classical Hodge Theory (cf. 3.1.2), every $\Lambda_{\varepsilon}$ is polarized (up to sign) by the intersection form, the space $\Lambda$ is semipolarized by the same form, i.e. the relevant bilinear form has a nontrivial radical, but it is positive semidefinite. At this point something quite remarkable happens: $\Lambda \subseteq H_{\leqslant 0}^{n}(X)$, i.e. $\Lambda=\Lambda_{\leqslant 0}$ and the radical of the semipolarization is $\Lambda_{\leqslant-1}=\Lambda \cap H_{\leqslant-1}^{n}(X)$. It follows that $\Lambda_{0}:=\Lambda_{\leqslant 0} / \Lambda_{\leqslant-1} \subseteq H_{0}^{n}(X)$ is polarized by $S_{00}^{\eta L}$. Finally, since $P_{0}^{0} \subseteq \Lambda_{0}$ is a Hodge sub-structure, it is automatically polarized (cf. 3.1.1).

- Step 5. The Generalized Grauert Contractibility Criterion 2.1.9 is proved in 6.3 in the context of proving the semisimplicity of ${ }^{p} \mathcal{H}^{0}\left(f_{*} \mathbb{Q}_{X}[n]\right)$, i.e. the remaining case of Theorem 2.1.1(c). By slicing the strata, we reduce the proof to the key case when the perversity index is zero and the stratum is zero-dimensional. To deal with this case we use Deligne's Theory of Mixed Hodge structures to infer the injectivity part of the statement. The relevant graded piece of the homology of the fiber is then a Hodge sub-structure of the corresponding perverse cohomology group of $X$, compatibly with the $(\eta, L)$ Decomposition. The nondegeneration statement follows from the Generalized HodgeRiemann Bilinear Relations and the elementary 3.1.1.

- Step 6. We prove that ${ }^{p} \mathcal{H}^{0}\left(f_{*} \mathbb{Q}_{X}[n]\right)$ is semisimple in Section 6. We first prove Theorem 6.3.2 which states that the complex in question is a direct sum of intersection cohomology complexes of local systems on strata. The proof uses the Splitting Criterion 4.1.3: 
the criterion is met by virtue of the Generalized Grauert Contractibility Criterion. The Refined Intersection Form Theorem 2.1.10 follows from Theorem 6.3.2 and the Splitting Criterion 4.1.3. We prove Theorem 6.4.2, i.e. that the local systems above are semisimple by exhibiting them as quotients of local systems associated with certain auxiliary smooth proper maps.

The Purity Theorem 2.2.1 is proved in 7.1 by a similar induction, using the results of 2.1 as well as Deligne's theory of Mixed Hodge structures.

\section{Notation and preliminary results}

We work over $\mathbb{C}$ and denote rational singular cohomology groups by $H^{*}(-)$.

\subsection{The topology and Hodge Theory of algebraic varieties}

In this section we collect classical results concerning the topology and the Hodge theory of projective manifolds. For a comprehensive introduction to Hodge theory and its applications to Algebraic Geometry, see [32].

Let $l \in \mathbb{Z}, H$ be a finitely generated abelian group, $H_{\mathbb{Q}}:=H \otimes_{\mathbb{Z}} \mathbb{Q}, H_{\mathbb{R}}=H \otimes_{\mathbb{Z}} \mathbb{R}, H_{\mathbb{C}}=$ $H \otimes_{\mathbb{Z}} \mathbb{C}$.

A pure Hodge structure of weight $l$ on $H, H_{\mathbb{Q}}$ or $H_{\mathbb{R}}$, is a direct sum decomposition $H_{\mathbb{C}}=$ $\bigoplus_{p+q=l} H^{p q}$ such that $H^{p q}=\overline{H^{q p}}$. The Hodge filtration is the decreasing filtration $F^{p}\left(H_{\mathbb{C}}\right):=$ $\bigoplus_{p^{\prime} \geqslant p} H^{p^{\prime} q^{\prime}}$. A morphism of Hodge structures $f: H \rightarrow H^{\prime}$ is a group homomorphism such that $f \otimes \mathrm{Id}_{\mathbb{C}}$ is compatible with the Hodge filtration, i.e. such that it is a filtered map. Such maps are automatically strict. The category of Hodge structures of weight $l$ is abelian.

Let $C$ be the Weil operator, i.e. $C: H_{\mathbb{C}} \simeq H_{\mathbb{C}}$ is such that $C(x)=i^{p-q} x$, for every $x \in H^{p q}$. It is a real operator. Replacing $i^{p-q}$ by $z^{p} \bar{z}^{q}$ we get a real action $\rho$ of $\mathbb{C}^{*}$ on $H_{\mathbb{R}}$. A polarization of the real pure Hodge structure $H_{\mathbb{R}}$ is a real bilinear form $\Psi$ on $H_{\mathbb{R}}$ which is invariant under the action given by $\rho$ restricted to $S^{1} \subseteq \mathbb{C}$ and such that the bilinear form $\widetilde{\Psi}(x, y):=\Psi(x, C y)$ is symmetric and positive definite. If $\Psi$ is a polarization, then $\Psi$ is symmetric if $l$ is even, and antisymmetric if $l$ is odd. In any case, $\Psi$ is nondegenerate. In addition, for every $0 \neq x \in H^{p q}$, $(-1)^{l} i^{p-q} \Psi(x, \bar{x})>0$, where $\Psi$ also denotes the $\mathbb{C}$-bilinear extension of $\Psi$ to $H_{\mathbb{C}}$. The following remark is used several times in this paper.

Remark 3.1.1. - If $H^{\prime} \subseteq H$ is a Hodge sub-structure, then $H_{\mathbb{R}}$ is fixed by $C$. It follows that $\Psi_{\mid H_{\mathbb{R}}^{\prime}}$ is a polarization, hence it is nondegenerate.

Let $X$ be a nonsingular projective variety of dimension $n, \eta$ be an ample line bundle on $X$. For every $r \geqslant 0$ define $P^{n-r}=\operatorname{Ker} \eta^{r+1} \subseteq H^{n-r}(X, \mathbb{Q})$. Classical Hodge Theory states that, for every $l, H^{l}(X, \mathbb{Z})$ is a pure Hodge structure of weight $l, P^{n-r}$ is a rational pure Hodge structure of weight $n-r$ polarized by a modification of the Poincaré pairing on $X$.

THEOREM 3.1.2.-

(a) (The Hard Lefschetz Theorem). For every $r \geqslant 0$ one has

$$
\eta^{r}: H^{n-r}(X, \mathbb{Q}) \simeq H^{n+r}(X, \mathbb{Q}) .
$$

(b) (The Primitive Lefschetz Decomposition). For every $r \geqslant 0$ there is the direct sum decomposition

$$
H^{n-r}(X, \mathbb{Q})=\bigoplus_{j \geqslant 0} \eta^{j} P^{n-r-2 j}
$$

$4^{\text {e }}$ SÉRIE - TOME $38-2005-\mathrm{N}^{\circ} 5$ 
where each summand is a pure Hodge sub-structure of weight $n-r$ and all summands are mutually orthogonal with respect to the bilinear form $\int_{X} \eta^{r} \wedge-\wedge-$.

(c) (The Hodge-Riemann Bilinear Relations). For every $0 \leqslant l \leqslant n$, the bilinear form $(-1)^{\frac{l(l+1)}{2}} \int_{X} \eta^{n-l} \wedge-\wedge-i$ s a polarization of the pure weight-l Hodge structure $P^{l} \subseteq$ $H^{l}(X, \mathbb{R})$. In particular,

$$
(-1)^{l(l-1) / 2} i^{p-q} \int_{X} \eta^{n-l} \wedge \alpha \wedge \bar{\alpha}>0, \quad \forall 0 \neq \alpha \in P^{l} \cap H^{p q}(X, \mathbb{C}) .
$$

A local system $\mathcal{L}$ on an algebraic variety $Y$ is said to be semisimple if every local subsystem $\mathcal{L}^{\prime}$ of $\mathcal{L}$ admits a complement, i.e. a local subsystem $\mathcal{L}^{\prime \prime}$ of $\mathcal{L}$ such that $\mathcal{L} \simeq \mathcal{L}^{\prime} \oplus \mathcal{L}^{\prime \prime}$.

Remark 3.1.3. - If $Y$ is normal and $Y^{\prime} \subseteq Y$ is a Zariski-dense open subset, then $\mathcal{L}$ is semisimple if and only $\mathcal{L}_{\mid Y^{\prime}}$ is semisimple. In fact, the natural map $\pi_{1}\left(Y^{\prime}, y^{\prime}\right) \rightarrow \pi_{1}\left(Y, y^{\prime}\right)$ is surjective for any $y^{\prime} \in Y^{\prime}$.

THEOREM 3.1.4 (Decomposition Theorem for proper smooth maps). - Let $f: X^{n} \rightarrow Y^{m}$ be a smooth proper map of smooth algebraic varieties of the indicated dimensions and $\eta$ be an ample line bundle on $X$. Then

$$
\eta^{i}: R^{n-m-i} f_{*} \mathbb{Q}_{X} \simeq R^{n-m+i} f_{*} \mathbb{Q}_{X}, \quad \forall i \geqslant 0, \quad R f_{*} \mathbb{Q}_{X} \simeq \bigoplus_{i \geqslant 0} R^{i} f_{*} \mathbb{Q}_{X}[-i]
$$

and the local systems $R^{j} f_{*} \mathbb{Q}_{X}$ are semisimple on $Y$.

Proof. - See [9] and [11, Théorème 4.2.6].

THEOREM 3.1.5 (Mixed Hodge structure on cohomology). - Let $X$ be an algebraic variety. For each $j$ there is an increasing weight filtration

$$
\{0\}=W_{-1} \subseteq W_{0} \subseteq \cdots \subseteq W_{2 j}=H^{j}(X, \mathbb{Q})
$$

and a decreasing Hodge filtration

$$
H^{j}(X, \mathbb{C})=F^{0} \supseteq F^{1} \supseteq \cdots \supseteq F^{m} \supseteq F^{m+1}=\{0\}
$$

such that the filtration induced by $F^{\bullet}$ on the complexified graded pieces of the weight filtration endows every graded piece $W_{l} / W_{l-1}$ with a pure Hodge structure of weight $l$.

This structure is functorial for maps of algebraic varieties and the induced maps strictly preserve both filtrations.

We shall need the following two properties of this structure. See $[12,8.2]$ for more.

THEOREM 3.1.6. - Let $Z \subseteq U \subseteq X$ be embeddings where $U$ is a Zariski-dense open subvariety of the nonsingular variety $X$ and $Z$ is a closed subscheme of $X$. Then

$$
\operatorname{Im}\left(H^{j}(X) \rightarrow H^{j}(Z)\right)=\operatorname{Im}\left(H^{j}(U) \rightarrow H^{j}(Z)\right), \quad \forall j \geqslant 0 .
$$

Let $g: T \rightarrow Z$ be a proper algebraic map of proper schemes, $T$ nonsingular. Then

$$
\operatorname{Ker}\left(g^{*}: H^{j}(Z) \rightarrow H^{j}(T)\right)=W_{j-1}\left(H^{j}(Z)\right), \quad \forall j \geqslant 0 .
$$




\subsection{Whitney stratifications of algebraic maps}

The recent book [14] provides an accessible introduction to many of the topics treated in this and in the following sections. It is known that every algebraic variety $Y$ of dimension $d$ admits a Whitney stratification $\mathfrak{Y}$ where the strata are locally closed algebraic subsets with a finite number of irreducible nonsingular components. See [2, I.1, I.4], [17, I] and the references contained therein. In particular, $Y$ admits a filtration $Y=Y_{d} \supseteq Y_{d-1} \supseteq Y_{d-2} \supseteq \cdots \supseteq Y_{1} \supseteq Y_{0} \supseteq Y_{-1}=\emptyset$ by closed algebraic subsets subject to the following properties.

(1) $S_{l}:=Y_{l} \backslash Y_{l-1}$ is either empty or a locally closed algebraic subset of pure dimension $l$; the connected components of $S_{l}$ are a finite number of nonsingular algebraic varieties. We have Zariski-dense open sets $U_{l}:=Y \backslash Y_{l-1}=\bigsqcup_{l^{\prime} \geqslant l} S_{l^{\prime}}$, such that $U_{l}=U_{l+1} \amalg S_{l}$. Note that $U_{d}$ is a nonsingular Zariski-dense open subset of $Y$ and that $U_{0}=Y$.

(2) (Local normal triviality) Let $y \in S_{l}, \bar{N}$ be a normal slice through $S_{l}$ at $y, \mathfrak{L}$ be the link of $S$ at $y, N:=\bar{N} \backslash \mathfrak{L}$ be the (open) normal slice. The spaces $\bar{N}, \mathfrak{L}$ and $N$ inherit Whitney stratifications. $\bar{N}$ (N, respectively) is homeomorphic in a stratum-preserving manner to the cone $c(\mathfrak{L})(c(\mathfrak{L}) \backslash \mathfrak{L}$, respectively) over the link $\mathfrak{L}$ with vertex identified to $y$. The cone is stratified using the cone structure and the given stratification of the link. The point $y$ admits an open euclidean neighborhood $W$ in $Z$ which is homeomorphic in a stratumpreserving manner to $\mathbb{C}^{l} \times N$.

DEFINITION 3.2.1 (Stratification of $Y$ ). - In this paper, the term stratification of $Y$ indicates a finite, algebraic Whitney stratification of $Y$. The resulting open and closed embeddings are denoted by $S_{l} \stackrel{\alpha_{l}}{\longrightarrow} U_{l} \stackrel{\beta_{l}}{\longleftarrow} U_{l+1}$.

Remark 3.2.2. - Let $Y \subseteq \mathbb{P}^{N}$ be a quasi-projective variety, $\mathfrak{Y}$ a stratification of $Y$. Bertini Theorem implies that, for every $l>0$ for which $S_{l}$ is not empty, the normal slice $N$ through a point $y \in S_{l}$ can be chosen to be the trace, in a suitable euclidean neighborhood of $y$ in $Y$, of a complete intersection of $l$ hyperplanes of $\mathbb{P}^{N}$ passing through $y$, transversal to all strata of $\mathfrak{Y}$.

The Thom Isotopy Lemmas, adapted to the algebraic setting, yield the following result. See [17, I.7].

THEOREM 3.2.3. - Let $f: X \rightarrow Y$ be an algebraic map of algebraic varieties. There exist finite algebraic Whitney stratifications $\mathfrak{X}$ of $X$ and $\mathfrak{Y}$ of $Y$ such that, given any connected component $S$ of a $\mathfrak{Y}$ stratum $S_{l}$ on $Y$ :

(1) $f^{-1}(S)$ is an union of connected components of strata of $\mathfrak{X}$ each of which is mapping submersively to $S$; in particular, every fiber $f^{-1}(y)$ is stratified by its intersection with the strata of $\mathfrak{X}$.

(2) $\forall y \in S$ there exists an euclidean open neighborhood $U$ of $y$ in $S$ and a stratum-preserving homeomorphism $h: U \times f^{-1}(y) \simeq f^{-1}(U)$ such that $f \circ h$ is the projection to $U$.

DEFINITION 3.2.4 (Stratification for $f$ ). - A pair of stratifications $\mathfrak{X}$ and $\mathfrak{Y}$ as in Theorem 3.2.3 is called a stratification for $f$.

If $f$ is an open immersion, then a stratification $\mathfrak{Y}$ induces one on $X$. If $f$ is a closed immersion, one can choose a finite Whitney stratification $\mathfrak{X}$ so that every stratum of it is the intersection of $X$ with strata of $\mathfrak{Y}$ of the same dimension. In either case, one obtains a stratification for $f$.

\subsection{The category $D(Y)$}

Let $Y$ be an algebraic variety and $D^{b}(Y)$ be the bounded derived category of sheaves of rational vector spaces on $Y$. We refer to [2, $\S \mathrm{V}]$ and to [19] for an account of the formalism of derived categories and Poincaré-Verdier Duality.

$4^{\text {e }}$ SÉRIE - TOME $38-2005-\mathrm{N}^{\circ} 5$ 
DEFINITION 3.3.1 (Cohomologically-constructible). - Let $\mathfrak{Y}$ be stratification of $Y$. We say that $K \in O b\left(D^{b}(Y)\right)$ is $\mathfrak{Y}$-cohomologically-constructible (in short, $\mathfrak{Y}$-cc) if $\forall j \in \mathbb{Z}$ and $\forall l$, the sheaves $\mathcal{H}^{j}(K)_{\mid S_{l}}$ are locally constant and the stalks are finite dimensional.

Let $D(Y)$ be the full sub-category of $D^{b}(Y)$ consisting of those complexes $K$ which are $\mathfrak{Y}$-cc with respect to some stratification $\mathfrak{Y}$ of $Y$.

The category $D(Y)$ is triangulated and it is preserved by the truncation, Verdier Duality and Rhom functors. The dualizing complex of $Y$ is denoted by $\omega_{Y} \in O b(D(Y))$ and the Verdier dual of a complex $K$ by $\mathcal{D}(K)=\operatorname{Rhom}\left(K, \omega_{Y}\right)$. In fact, $\mathfrak{Y}$-cc complexes are stable under these constructions. By triangle we mean a distinguished triangle.

The four functors $\left(R f_{!}, f^{!}, f^{*}, R f_{*}\right)$, are denoted here simply by $\left(f_{!}, f^{!}, f^{*}, f_{*}\right)$.

One has the following properties; see [2, V.10.13 and 16]: if $F$ is $\mathfrak{Y}$-cc and $G$ is $\mathfrak{X}$-cc, then $f^{*} F, f^{!} F$ are $\mathfrak{X}$-cc and $f_{*} G$ and $f_{!} G$ are $\mathfrak{Y}$-cc. In particular, $f_{!} f^{!} F, f_{*} f^{*} F$ are $\mathfrak{Y}$-cc and $f^{!} f_{!} G$, $f^{*} f_{*} G$ are $\mathfrak{X}$-cc.

The following facts are used in the sequel, often without explicit mention.

The pairs $\left(f_{!}, f^{!}\right)$and $\left(f^{*}, f_{*}\right)$ are pairs of adjoint functors so that there are natural transformations Id $\rightarrow f_{*} f^{*}, f^{*} f_{*} \rightarrow \mathrm{Id}, f_{!} f^{!} \rightarrow \mathrm{Id}$ and Id $\rightarrow f^{!} f_{!}$.

Let $\alpha: Z \rightarrow Y$ be the embedding of a closed algebraic subset, $\beta: U \rightarrow Y$ be the embedding of the open complement and $K \in O b(D(Y))$. There are natural isomorphism: $\alpha_{!} \simeq \alpha_{*}, \beta^{!} \simeq \beta^{*}$ and dual triangles:

$$
\alpha ! \alpha ! K \rightarrow K \rightarrow \beta_{*} \beta^{*} K \stackrel{[1]}{\longrightarrow}, \quad \beta_{!} \beta^{!} K \rightarrow K \rightarrow \alpha_{*} \alpha^{*} K \stackrel{[1]}{\longrightarrow},
$$

whose associated long exact sequences in hypercohomology are the ones of the pairs $\mathbb{H}^{l}(Y, U, K)$ and $\mathbb{H}^{l}(Y, Z, K)$, respectively.

There are canonical isomorphisms $f^{!} \omega_{Y} \simeq \omega_{X}, \mathcal{D D} \simeq \operatorname{Id}_{D(Y)}, \mathcal{D} f_{*} \simeq f_{!} \mathcal{D}$ and $\mathcal{D} f^{*} \simeq f^{!} \mathcal{D}$.

Let $f$ be proper and consider a Cartesian diagram

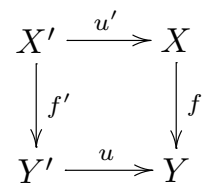

Since $f_{!} \simeq f_{*}$, the Change of Coefficients Formula reads:

$$
K \stackrel{\mathbb{L}}{\otimes} f_{*} K^{\prime} \simeq f_{*}\left(f^{*} K \stackrel{\mathbb{L}}{\otimes} K^{\prime}\right), \quad \forall K \in O b(D(Y)), \forall K^{\prime} \in O b(D(X))
$$

and the Base Change Theorem for Proper Maps reads:

$$
u^{*} f_{*} \simeq f^{\prime}{ }_{*}^{\prime *}, \quad u^{!} f_{*} \simeq f_{*}^{\prime} u^{\prime !} .
$$

\subsection{The intersection form on the fibers of the map $f: X \rightarrow Y$}

In this section we introduce the intersection form on the fibers of an algebraic map $f: X \rightarrow Y$. We could not find a reference serving the needs of the present paper. Lemma 4.3.6 and Theorem 2.1.10 describe important properties of the intersection form.

Let $Z$ be an algebraic set, $c: Z \rightarrow p t$ be the constant map. We have $\omega_{Z} \simeq c^{\prime} \mathbb{Q}_{p t}$. Define the Borel-Moore homology groups with rational coefficients of $Z$ as

$$
H_{l}^{\mathrm{BM}}(Z):=\mathbb{H}^{-l}\left(Z, \omega_{Z}\right)
$$


We have that $H_{l}^{\mathrm{BM}}(Z) \simeq H_{c}^{l}(Z)^{\vee}$. If $Z$ is compact, then $H_{l}^{\mathrm{BM}}(Z) \simeq H_{l}(Z)$. Let $i: Z \rightarrow W$ be a map of algebraic sets. If $i$ is proper, then the natural adjunction map, the identification $i_{*} \simeq i_{\text {! }}$ and the isomorphism, $\omega_{Z} \simeq i^{!} \omega_{W}$, give the map $i_{*} \omega_{Z} \rightarrow \omega_{W}$. The resulting maps in hypercohomology $i_{*}: H_{l}^{\mathrm{BM}}(Z) \rightarrow H_{l}^{\mathrm{BM}}(W)$ are the usual proper-push-forward maps. If $i$ is an open immersion, then using the natural adjunction map and the identification $i^{*} \simeq i^{!}$, we get a map $\omega_{W} \rightarrow i_{*} \omega_{Z}$ whose counterparts in hypercohomology are the restriction to an open set maps $H_{l}^{\mathrm{BM}}(W) \rightarrow H_{l}^{\mathrm{BM}}(Z)$.

Let $y \in Y$ and $i: f^{-1}(y) \rightarrow X$. Using the isomorphism $\omega_{X}[-n] \simeq \mathbb{Q}_{X}[n]$, we get a natural sequence of maps

$$
i_{!} \omega_{f^{-1}(y)}[-n] \rightarrow \omega_{X}[-n] \simeq \mathbb{Q}_{X}[n] \rightarrow i_{*} \mathbb{Q}_{f^{-1}(y)}[n]
$$

where the first and third map are each other's dual. Taking degree $l$ hypercohomology we get maps

$$
H_{n-l}^{\mathrm{BM}}\left(f^{-1}(y)\right) \rightarrow H_{n-l}^{\mathrm{BM}}(X) \simeq H^{n+l}(X) \rightarrow H^{n+l}\left(f^{-1}(y)\right) .
$$

The resulting pairing

$$
H_{n-l}^{\mathrm{BM}}\left(f^{-1}(y)\right) \times H_{n+l}\left(f^{-1}(y)\right) \rightarrow \mathbb{Q}
$$

is called the refined intersection form on $f^{-1}(y) \subseteq X$. Note that we may replace $X$ by any euclidean open neighborhood of $f^{-1}(y)$. Geometrically, this form corresponds to intersecting locally finite cycles supported on $f^{-1}(y)$ with finite cycles of complementary dimension in $X$ supported on $f^{-1}(y)$.

Remark 3.4.1. - If $y$ lies on a positive dimensional stratum $S_{l}, l>0$, then the refined intersection form is trivial: in fact, by the local triviality of the stratification, a cycle supported on $f^{-1}(y)$ can be moved to a homologous one supported on a nearby fiber $f^{-1}\left(y^{\prime}\right), y^{\prime} \neq y$, $y^{\prime} \in S_{l}$. See also 2.1.11.

We now assume that $f$ is proper. We have the canonical identification $H_{l}^{\mathrm{BM}}\left(f^{-1}(y)\right) \simeq$ $H_{l}\left(f^{-1}(y)\right)$ and the usual base-change identifications:

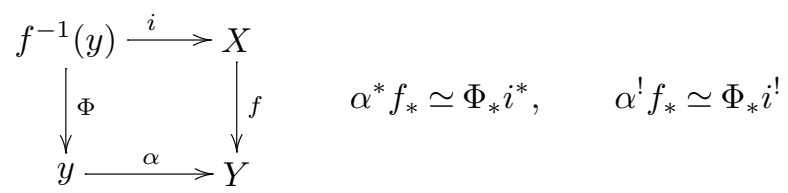

giving rise to a self-dual diagram of adjunction maps:

$$
\alpha_{!} \alpha^{!} f_{*} \omega_{X}[-n] \rightarrow f_{*} \omega_{X}[-n] \simeq f_{*} \mathbb{Q}_{X}[n] \rightarrow \alpha_{*} \alpha^{*} f_{*} \mathbb{Q}_{X}[n]
$$

which, after taking hypercohomology, give the refined intersection form (10) on $f^{-1}(y)$.

Remark 3.4.2. - We shall consider the map $\alpha ! \alpha^{!} f_{*} \mathbb{Q}_{X}[n] \rightarrow f_{*} \mathbb{Q}_{X}[n]$ in connection with the Splitting Criterion 4.1.3. On the other hand, the map that arises geometrically is $\alpha_{!} \alpha^{!} f_{*} \omega_{X}[-n] \rightarrow f_{*} \omega_{X}[-n]$. Using that $\alpha_{!} \alpha^{!} \rightarrow \mathrm{Id}$ is a natural transformation of additive 
functors and the isomorphism $\omega_{X}[-n] \simeq \mathbb{Q}_{X}[n]$, one has a commutative diagram:

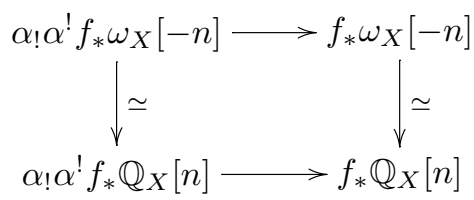

The two horizontal maps are thus equivalent and one can check the hypotheses of the Splitting Criterion 4.1.3 on the top one.

\subsection{The local structure of a $\mathfrak{Y}$-cc complex along a stratum}

The references are [17, 1.4], [2, V.3. and Lemma V.10.14]. Let $Y$ be a projective variety, $\mathfrak{Y}$ be a stratification, $y \in S \subseteq S_{l}$ be a point in a connected component $S$ of a stratum $S_{l}, N$ be a normal slice through $S$ at $y$. Let $W$ be a standard open neighborhood of $y$ in $Y$, homeomorphic in a stratum-preserving manner to $\mathbb{C}^{l} \times N$. Let $\pi: W \rightarrow N$ be the corresponding map, $\dot{N}:=N \backslash y$, $\dot{W}:=W \backslash(S \cap W)$. We have a commutative diagram of Cartesian squares, where $a, \alpha, i_{y}$, and $i_{N}$ are closed immersions, $b$ and $\beta$ are open immersions, $c, \pi$ and $\dot{\pi}$ are trivial topological $\mathbb{C}^{l}$-bundles, $c \circ \alpha_{y}=\operatorname{Id}_{y}$ and $\pi \circ i_{N}=\operatorname{Id}_{N}$ :

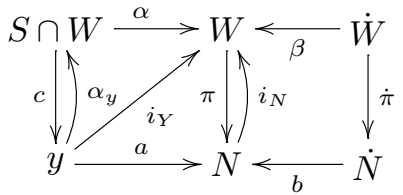

The following rules apply: $\alpha_{*} \simeq \alpha_{!}, \alpha^{*} \alpha_{*} \simeq \mathrm{Id} \simeq \alpha^{!} \alpha_{*}, \pi^{*} \simeq \pi^{!}[-2 l], c^{*} \simeq c^{!}[-2 l]$, $\beta_{*} \dot{\pi}^{*} \simeq \pi^{*} b_{*}$. If $K$ has locally constant cohomology sheaves on $S$, then $\alpha_{y}^{!} K \simeq \alpha_{y}^{*} K[-2 l]$.

Let $K$ be $\mathfrak{Y}$-cc. On $W$, we have that: $K \simeq \pi^{*} \pi_{*} K \simeq \pi^{*} K_{\mid N}$. That is, $K$ has, locally at a point of any stratum, a product structure along the stratum. See [2, Lemma V.10.14].

The sheaves $\mathcal{H}^{i}\left(\alpha^{*} K\right)$ and $\mathcal{H}^{i}\left(\alpha^{!} K\right)$ on $S \cap W$ are constant with representative stalks $\mathcal{H}^{i}\left(\alpha^{*} K\right)_{y} \simeq \mathbb{H}^{i}\left(N, K_{\mid N}\right) \simeq \mathcal{H}^{i}\left(a^{*} K_{\mid N}\right)$, and $\mathcal{H}^{i}(\alpha ! K)_{y} \simeq \mathbb{H}^{i}\left(N, \dot{N} ; K_{\mid N}\right) \simeq \mathcal{H}^{i}\left(a^{!} K_{\mid N}\right)$.

Remark 3.5.1. - Since $\pi^{*}$ is fully faithful, if $K$ is self-dual, then $K_{\mid N}[-l]$ is self-dual. If $K$ is perverse (cf. 3.6) on $Y$, then, using the characterization of perverse sheaves in Remark 3.6.1 and Lemma 3.5.4, one shows that $K_{\mid N}[-l]$ is perverse on $N$.

Remark 3.5.2. - Let $\alpha: Y^{\prime} \rightarrow Y$ be a closed immersion of algebraic varieties. We have that $\alpha ! \simeq \alpha_{*}$ are fully faithful so that for every $K \in O b(D(Y))$, the composition of the adjunction maps $\alpha_{!} \alpha^{!} K \rightarrow K \rightarrow \alpha_{*} \alpha^{*} K$ yields a natural map: $\alpha^{!} K \rightarrow \alpha^{*} K$.

LEMMA 3.5.3. - Let $Y$ be an algebraic variety, $\mathfrak{Y}$ be a stratification of $Y, K$ be $\mathfrak{Y}$-cc, $\alpha: S \rightarrow Y$ be the embedding of a connected component of a stratum $S_{l}, y \in S$.

The natural map $\alpha^{!} K \rightarrow \alpha^{*} K$ coincides, when restricted to a standard neighborhood $W$ of $y$ in $Y$, with $c^{*}$ of the analogous map $a^{!} K_{\mid N} \rightarrow a^{*} K_{\mid N}$.

The same is true for the induced maps $\mathcal{H}^{j}\left(\alpha^{!} K\right) \rightarrow \mathcal{H}^{j}\left(\alpha^{*} K\right), \mathcal{H}^{j}\left(\alpha^{!} K\right)_{y} \rightarrow \mathcal{H}^{j}\left(\alpha^{*} K\right)_{y}$ induced on the cohomology sheaves and on their stalks at $y$.

Finally: $\mathcal{H}^{j}\left(\alpha^{!} K\right)_{y} \simeq \mathcal{H}^{-j-2 l}\left(i_{y}^{*} \mathcal{D}(K)\right)^{\vee} \simeq \mathcal{H}^{-j}\left(a^{*} \mathcal{D}\left(K_{\mid N}\right)\right)^{\vee}$. 
Proof. - The question being local around $y \in Y$, we may work on $W$. We may assume that $K=\pi^{*} K_{\mid N}$ and that $S$ is closed, so that, since $\alpha_{!} \simeq \alpha_{*}$ are fully faithful, it is enough to study the map $\alpha^{!} K \rightarrow \alpha^{*} K$.

We have $\alpha^{!} \pi^{*} K_{\mid N} \simeq \alpha^{!} \pi^{!} K_{\mid N}[-2 s] \simeq c^{!} a^{!} K_{\mid N}[-2 s] \simeq c^{*}\left(a^{!} K_{\mid N}\right)$, i.e. $\left(\alpha_{!} \alpha^{!} K\right)_{\mid S}$ is a pullback from $p$ and so are its cohomology sheaves. The statement concerning $\alpha ! K \rightarrow \alpha^{*} K$, the induced maps on the cohomology sheaves and associated stalks at $y$ follow. The duality statements stem from Poincaré-Verdier Duality on $y$ and the isomorphism $\alpha_{y}^{*} \alpha^{!} K \simeq i_{y}^{!} K[2 l]$, which holds in view of the fact that $\alpha ! K$ has locally constant cohomology sheaves on $S$.

A global counterpart of a normal slice is the notion of stratified normally nonsingular inclusion; see [17, Theorem I.1.11]. The embedding $Z \rightarrow Y$ of a subvariety is said to be a normally nonsingular inclusion if there exists a neighborhood $W$ of $Z$ in $Y$ and a retraction $\pi: W \rightarrow Z$ which is locally homeomorphic to a projection: every point $z \in Z$ has a neighborhood $U \subseteq Z$ and a homeomorphism $\pi^{-1}(U) \simeq U \times \mathbb{C}^{l}$ compatible with the maps to $U$. In addition, the homeomorphism $\pi^{-1}(U) \simeq U \times \mathbb{C}^{l}$ can be chosen so that it is stratum-preserving with respect to the induced stratification on $\pi^{-1}(U)$ and to the stratification on $U \times \mathbb{C}^{l}$ given by the product of the trivial stratification on $\mathbb{C}^{l}$ with that induced on $U$ by the transversality assumption.

A normally nonsingular inclusion can produced by intersecting a projective variety $Y$ with a subvariety of the ambient projective space, e.g. a hypersurface, which intersects transversally every stratum of a given stratification $\mathfrak{Y}$ of $Y$ (cf. 3.2.2). The universal hyperplane section construction in 4.7 is an example. A normally nonsingular inclusion carries a cohomology class.

The following fact is well-known and will be used often in this paper.

LEMMA 3.5.4. - Let $i: Z \rightarrow Y$ be a normally nonsingular inclusion of complex codimension $d$ of complex varieties, transversal to every stratum of a stratification $\mathfrak{Y}$ of $Y$, and $K$ be $\mathfrak{Y}$-cc. Let $\pi: W \rightarrow Z$ be a retraction of a tubular neighborhood of $Z$ in $Y$ onto $Z$. Then we have (a) $K_{\mid W} \simeq \pi^{*} \pi_{*}\left(K_{\mid W}\right) \simeq \pi^{*} K_{\mid Z}$ and (b) $i^{!} K \simeq i^{*} K[-2 d]$.

Proof. - We denote $K_{\mid W}$ simply by $K$.

(a) By virtue of the local triviality assumption, the natural adjunction map $\pi^{*} \pi_{*}(K) \rightarrow K$ is an isomorphism by [2, Lemma V.10.14]. The second isomorphism follows from the first one and the identification $i^{*} \pi^{*} \simeq \operatorname{Id}_{Z}^{*}: \pi^{*} \pi_{*} K \simeq \pi^{*}\left(i^{*} \pi^{*}\right) \pi_{*} K \simeq \pi^{*} i^{*} K=\pi^{*} K_{\mid Z}$.

(b) We use the natural identifications $\mathcal{D}^{2} \simeq \mathrm{Id}, i^{!} \simeq \mathcal{D}_{Z} i^{*} \mathcal{D}_{W}$ and the fact that $\pi^{!} \simeq \pi^{*}[2 d]$, for $\pi$ is a locally trivial $\mathbb{C}^{d}$-bundle. Denote the dualizing complexes of $W$ and $Z$ by $\omega_{W}$ and $\omega_{Z}$. One has $\omega_{W} \simeq \pi^{!} \omega_{Z}$. We have $i^{!} K \simeq \mathcal{D}_{Z} i^{*} \operatorname{Rhom}\left(K, \omega_{W}\right) \simeq \mathcal{D}_{Z} \operatorname{Rhom}\left(i^{*} K, i^{*} \pi^{!} \omega_{Z}\right) \simeq$ $\left(\mathcal{D}_{Z} \operatorname{Rhom}\left(i^{*} K, i^{*} \pi^{*} \omega_{Z}\right)\right)[-2 d] \simeq\left(\mathcal{D}_{Z} \operatorname{Rhom}\left(i^{*} K, \omega_{Z}\right)\right)[-2 d] \simeq \mathcal{D}^{2}\left(i^{*} K\right)[-2 d]$.

\subsection{Perverse sheaves}

Let $Y$ be an algebraic variety. We consider the $t$-structure on $D(Y)$ associated with the middle perversity see [1], [21, §10]. The associated heart is denoted by $\operatorname{Perv}(Y)$ and it is a full abelian sub-category of $D(Y)$. Its objects are called perverse sheaves, despite the fact that they are complexes. In short, we have the following structure.

- Two full sub-categories $D^{\leqslant 0}(Y)$ and $D^{\geqslant 0}(Y)$ of $D(Y)$ :

$$
\begin{aligned}
& O b\left(D^{\leqslant 0}(Y)\right)=\left\{K \in D(Y) \mid \operatorname{dimsupp} \mathcal{H}^{j}(K) \leqslant-j, \forall j\right\}, \\
& O b\left(D^{\geqslant 0}(Y)\right)=\left\{K \in D(Y) \mid \operatorname{dimsupp} \mathcal{H}^{j}(\mathcal{D}(K)) \leqslant-j, \forall j\right\} .
\end{aligned}
$$

Set $D^{\leqslant m}(Y):=D^{\leqslant 0}(Y)[-m], D^{\geqslant m}(Y):=D^{\geqslant 0}(Y)[-m]$.

$4^{\mathrm{e}}$ SÉRIE - TOME $38-2005-\mathrm{N}^{\circ} 5$ 
Remark 3.6.1. - These conditions can be re-formulated using a stratification $\mathfrak{Y}$ as follows. Let $K$ be $\mathfrak{Y}$-cc and $\alpha_{l}: S_{l} \rightarrow Y$ be the corresponding embedding. We have:

$K \in O b\left(D^{\leqslant 0}(Y)\right)$ if and only if $\mathcal{H}^{j}\left(\alpha_{l}^{*} K\right)=0, \forall l$ and $j$ s.t. $j>-l$. This is known as the condition of support.

$F \in O b\left(D^{\geqslant 0}(Y)\right)$ if and only if $\mathcal{H}^{j}\left(\alpha_{l}^{!} F\right)=0, \forall l$ and $j$ s.t. $j<-l$. This is known as the condition of co-support.

- If $P \in \operatorname{Perv}(Y)$, then $\mathcal{H}^{i}(P)=0$ for $i \notin[-\operatorname{dim} Y, 0]$. More precisely, if $P$ is $\mathfrak{Y}$-cc and $0 \leqslant s \leqslant d$, then $\mathcal{H}^{i}\left(P_{\mid U_{s}}\right)=0$ for $i \notin[-\operatorname{dim} Y,-s]$.

If $P \in \operatorname{Perv}(Y)$ is $\mathfrak{Y}$-cc and is supported on a closed $s$-dimensional stratum $S_{s}$, then $P \simeq \mathcal{H}^{-s}(P)[s]$.

- If $F \in O b\left(D^{\leqslant m}(Y)\right)$ and $G \in O b\left(D^{\geqslant m+t}(Y)\right)$ for $t>0$, then

$$
\operatorname{Hom}_{D(Y)}(F, G)=0 .
$$

- There are the perverse truncations functors, defined up to unique isomorphism, ${ }^{p} \tau_{\leqslant m}: D(Y) \rightarrow D^{\leqslant m}(Y)$, and ${ }^{p} \tau_{\geqslant m}: D(Y) \rightarrow D^{\geqslant m}(Y)$, adjoint to the inclusion functors, that is

$$
\operatorname{Hom}_{D(Y)}(F, G)=\operatorname{Hom}_{D \leqslant m(Y)}\left(F,{ }^{p} \tau_{\leqslant m} G\right) \quad \text { if } F \in O b\left(D^{\leqslant m}(Y)\right)
$$

and

$$
\operatorname{Hom}_{D(Y)}(G, F)=\operatorname{Hom}_{D \geqslant m(Y)}\left({ }^{p} \tau_{\geqslant m} G, F\right) \quad \text { if } F \in O b\left(D^{\geqslant m}(Y)\right) .
$$

There are adjunction maps ${ }^{p}{ }_{\uparrow \leqslant m} K \rightarrow K$ and $K \rightarrow{ }^{p}{ }_{\tau_{\geqslant m}} K$. By the boundedness hypothesis on $D(Y),{ }^{p} \tau_{\leqslant m} K \simeq 0$ if $m \ll 0$ and ${ }^{p} \tau_{\geqslant m} K \simeq 0$ if $m \gg 0$.

- If $K$ is $\mathfrak{Y}$-cc, then so are ${ }^{p} \tau_{\leqslant m} K$ and ${ }^{p_{\tau}}{ }_{\geqslant m} K$.

- There are canonical isomorphisms of functors

$$
{ }^{p} \tau_{\leqslant m} \circ[l] \simeq[l] \circ{ }^{p} \tau_{\leqslant m+l}, \quad{ }^{p} \tau_{\geqslant m} \circ[l] \simeq[l] \circ{ }^{p} \tau_{\geqslant m+l} .
$$

- For every $K$ and $m$ there is a functorial triangle

$$
{ }^{p} \tau_{\leqslant m} K \rightarrow K \rightarrow{ }^{p} \tau_{\geqslant m+1} K \stackrel{[1]}{\longrightarrow} .
$$

- The heart $\operatorname{Perv}(Y):=D^{\leqslant 0}(Y) \cap D^{\geqslant 0}(Y)$ of the $t$-structure is an abelian category which objects are called perverse sheaves. An object $K$ of $D(Y)$ is perverse if and only if the two natural maps coming from adjunction ${ }^{p} \tau_{\leqslant 0} K \rightarrow K$ and $K \rightarrow{ }^{p}{ }_{\tau_{\geqslant 0}} K$ are isomorphisms.

- The functor

$$
{ }^{p} \mathcal{H}^{0}(-): D(Y) \rightarrow \operatorname{Perv}(Y), \quad{ }^{p} \mathcal{H}^{0}(K):={ }^{p} \tau_{\leqslant 0}{ }^{p} \tau_{\geqslant 0} K \simeq{ }^{p} \tau_{\geqslant 0}{ }^{p} \tau_{\leqslant 0} K,
$$

is cohomological. Define

$$
{ }^{p} \mathcal{H}^{i}(K):={ }^{p} \mathcal{H}^{0}(K[i]) .
$$

These functors are called the perverse cohomology functors.

Any triangle $K^{\prime} \rightarrow K \rightarrow K^{\prime \prime} \stackrel{[1]}{\longrightarrow}$ in $D(Y)$ gives a long exact sequence in $\operatorname{Perv}(Y)$ :

$$
\cdots \rightarrow{ }^{p} \mathcal{H}^{i}\left(K^{\prime}\right) \rightarrow{ }^{p} \mathcal{H}^{i}(K) \rightarrow{ }^{p} \mathcal{H}^{i}\left(K^{\prime \prime}\right) \rightarrow{ }^{p} \mathcal{H}^{i+1}\left(K^{\prime}\right) \rightarrow \cdots
$$


If $K$ is $\mathfrak{Y}$-cc, then so are ${ }^{p} \mathcal{H}^{i}(K), \forall i \in \mathbb{Z}$.

By boundedness and the Five Lemma, a map $\phi: K \rightarrow K^{\prime}$ is an isomorphism iff ${ }^{p} \mathcal{H}^{i}(\phi):{ }^{p} \mathcal{H}^{i}(K) \rightarrow{ }^{p} \mathcal{H}^{i}\left(K^{\prime}\right)$ is an isomorphism for every $i$.

- Poincaré-Verdier Duality exchanges $D^{\leqslant 0}(Y)$ with $D^{\geqslant 0}(Y)$ and fixes $\operatorname{Perv}(Y)$. There are canonical isomorphisms of functors

$$
{ }^{p} \tau_{\leqslant 0} \circ \mathcal{D} \simeq \mathcal{D} \circ{ }^{p} \tau_{\geqslant 0}, \quad{ }^{p} \tau_{\geqslant 0} \circ \mathcal{D} \simeq \mathcal{D} \circ{ }^{p} \tau_{\leqslant 0}, \quad \mathcal{D} \circ{ }^{p} \mathcal{H}^{j} \simeq{ }^{p} \mathcal{H}^{-j} \circ \mathcal{D} .
$$

\section{7. $t$-exactness}

A functor $T: D_{1} \rightarrow D_{2}$ of triangulated categories with $t$-structures is said to be left (right, respectively) $t$-exact if $T\left(D_{1}^{\geqslant 0}\right) \subseteq D_{2}^{\geqslant 0}\left(T\left(D_{1}^{\leqslant 0}\right) \subseteq D_{2}^{\leqslant 0}\right.$, respectively) and it is said to be $t$ exact if it is left and right $t$-exact. If $T$ is $t$-exact, then it preserves the hearts of the two categories. In particular, if $T$ is $t$-exact, then there is a natural isomorphism ${ }^{p} \mathcal{H}^{l}(T(K)) \simeq T\left({ }^{p} \mathcal{H}^{l}(K)\right)$, $K \in O b\left(D_{1}\right)$. See [1, especially §4], and [21, §10].

Let $f: X \rightarrow Y$ be an algebraic map of algebraic varieties. We consider the triangulated categories $D(X)$ and $D(Y)$ with their middle-perversity $t$-structure. Verdier Duality is an autoequivalence of categories. It exchanges $f^{!}$with $f^{*}, f !$ with $f_{*}$ and $D^{\leqslant 0}(-)$ with $D^{\geqslant 0}(-)$. Consequently, statements about the left (right, respectively) $t$-exactness of the four functors $\left(f_{!}, f^{!}, f^{*}, f_{*}\right)$ are equivalent to the analogous statements of right (left, respectively) $t$-exactness of the four functors $\left(f_{*}, f^{*}, f^{!}, f_{!}\right)$. Similarly, $f^{*}[j]$ is left $t$-exact if and only if $f^{!}[-j]$ is right $t$-exact, etc.

If $f$ is affine, then $f_{*}$ is right $t$-exact and $f_{!}$is left $t$-exact (cf. [1, 4.1.1]). This is a convenient re-formulation of the theorem on the cohomological dimension of affine spaces and it implies the Weak Lefschetz Theorem (cf. 4.7).

If $f$ is quasi-finite and affine, then $f_{*}$ and $f$ ! are $t$-exact. If $f$ is smooth of relative dimension $d$, then $f^{*}[d] \simeq f^{!}[-d]$ and they are $t$-exact. If, in addition, $f$ is surjective and with connected fibers, then the induced functor $f^{*}[d]: \operatorname{Perv}(Y) \rightarrow \operatorname{Perv}(X)$ is fully faithful.

\subsection{Intersection cohomology complexes, semisimple objects and intermediate extensions}

Recall that $P \in O b(\operatorname{Perv}(Y))$ is said to be simple if it has no nontrivial sub-objects and hence no nontrivial quotients. $P$ is said to be semisimple if it is isomorphic to a direct sum of simple objects. The category $\operatorname{Perv}(Y)$ is abelian, artinian, i.e. every $P \in \operatorname{Perv}(Y)$ admits a finite filtration by sub-objects whose successive quotients are simple, and noetherian, i.e. any increasing filtration of $P$ by sub-objects stabilizes.

Let $\beta: U \rightarrow Y$ be a Zariski-dense open subset of $Y$. Given $P \in O b(\operatorname{Perv}(U))$, there is an object $\beta_{! *} P \in \operatorname{Perv}(Y)$ with the property that it extends $P$ and it has no nontrivial sub-object and quotient supported on a closed subvariety of $Y \backslash U$. It is unique up to isomorphism and is called the intermediate extension of $P$. See $[1,1.4 .25,2.1 .9,2.1 .11]$.

Given any stratification $\mathfrak{Y}$ of $Y$ for which $Y \backslash U$ is a union of connected components of strata, $\beta_{!_{*}} P$ is, up to isomorphism, the unique extension $\widetilde{P}$ of $P$ in $D(Y)$ such that, given any connected component, $S \stackrel{i}{\longrightarrow} Y \backslash U$, of a stratum contained in $Y \backslash U$, we have $\mathcal{H}^{j}\left(i^{*} \widetilde{P}\right)=0, \forall j \geqslant-\operatorname{dim} S$ and $\mathcal{H}^{j}\left(i^{!} \widetilde{P}\right)=0, \forall j \leqslant-\operatorname{dim} S$.

Remark 3.8.1. - The intermediate extension $\beta_{!_{*}} P$ can be described explicitly in terms of stratifications and successive push-forwards and truncations: let $\mathfrak{Y}$ be a stratification of $Y$ inducing stratifications on $U$ and $Y \backslash U$ with respect to which $P$ is $\mathfrak{Y}_{U}$-cc. The construction is by induction on the strata: if $U=U_{l+1}$, then $\beta_{l ! *} P \simeq \tau_{\leqslant-l-1} \beta_{l *} P$.

$4^{\mathrm{e}}$ SÉRIE - TOME $38-2005-\mathrm{N}^{\circ} 5$ 
Let $\mathcal{L}$ be a local system on an open set $U$ contained in the regular part of $Y$. The intersection cohomology complex associated with $\mathcal{L}$ is $I C_{Y}(\mathcal{L}):=\beta_{! *}(\mathcal{L}[\operatorname{dim} Y]) \in \operatorname{Ob}(\operatorname{Perv}(Y))$.

The case of $\mathcal{L}=\mathbb{Q}_{U}$ is of particular interest and gives rise to the intersection cohomology complex $I C_{Y}$ of $Y$. The groups $\mathbb{H}^{j}\left(Y, I C_{Y}\right) \simeq I H^{\operatorname{dim} Y+j}(Y, \mathbb{Q})$ are the rational intersection cohomology groups of $Y$ (cf. [16]).

If $Y$ is smooth, or at least a rational homology manifold, then $I C_{Y} \simeq \mathbb{Q}_{Y}[\operatorname{dim} Y]$.

The complex $I_{Y}(\mathcal{L})$ is characterized, up to isomorphism, by the following conditions:

- $\mathcal{H}^{j}\left(I C_{Y}(\mathcal{L})\right)=0$; for all $j<-\operatorname{dim} Y$;

- $\mathcal{H}^{-\operatorname{dim} Y}\left(I_{Y}(\mathcal{L})_{\mid U}\right) \simeq \mathcal{L}$

- $\operatorname{dim} \operatorname{supp} \mathcal{H}^{j}\left(I C_{Y}(\mathcal{L})\right)<-j$, if $j>-\operatorname{dim} Y$;

- $\operatorname{dim} \operatorname{supp}\left(\mathcal{H}^{j}\left(\mathcal{D}\left(I C_{Y}(\mathcal{L})\right)\right)\right)<-j$, if $j>-\operatorname{dim} Y$.

The last two conditions can be re-formulated using stratifications as follows. Let $\mathfrak{Y}$ be a stratification with respect to which $I C_{Y}(\mathcal{L})$ is $\mathfrak{Y}$-cc, $\alpha_{l}: S_{l} \rightarrow Y$ be the embedding. We have:

$$
\begin{array}{llll}
\mathcal{H}^{j}\left(\alpha_{l}^{*} I C_{Y}(\mathcal{L})\right)=0, & \forall l \quad \text { and } \quad j>\operatorname{dim} Y \quad \text { s.t. } j \geqslant-l ; \\
\mathcal{H}^{j}\left(\alpha_{l}^{!} I C_{Y}(\mathcal{L})\right)=0, & \forall l \quad \text { and } \quad j>-\operatorname{dim} Y & \text { s.t. } j \leqslant-l .
\end{array}
$$

One has $\mathcal{D}\left(I C_{Y}(\mathcal{L})\right) \simeq I C_{Y}\left(\mathcal{L}^{\vee}\right)$. Given a closed subvariety $i: Y^{\prime} \rightarrow Y$ and a complex of type $I C_{Y^{\prime}}\left(\mathcal{L}^{\prime}\right) \in \operatorname{Perv}\left(Y^{\prime}\right)$, we denote $i_{*} I C_{Y^{\prime}}\left(\mathcal{L}^{\prime}\right)$ simply by $I C_{Y^{\prime}}\left(\mathcal{L}^{\prime}\right)$. It is an object of $\operatorname{Perv}(Y)$ satisfying the conditions above, with $Y^{\prime}$ replacing $Y$.

An object $P \in \operatorname{Perv}(Y)$ is simple if and only if $P \simeq I C_{Y^{\prime}}\left(\mathcal{L}^{\prime}\right)$, for some closed subvariety $Y^{\prime} \subseteq Y$ and some simple local system $\mathcal{L}^{\prime}$ defined on an open subvariety of the regular part of $Y^{\prime}$. A semisimple $P$ is a finite direct sum of such objects.

Remark 3.8.2. - If $Y^{\prime}$ and $Y^{\prime \prime}$ are distinct, then the properties of intermediate extensions imply that $\operatorname{Hom}_{D(Y)}\left(I C_{Y^{\prime}}\left(L^{\prime}\right), I C_{Y^{\prime \prime}}\left(L^{\prime \prime}\right)\right)=0$.

\section{Preparatory results}

In this section we collect a series of results needed in the sequel for which we could not find an adequate reference.

\subsection{A splitting criterion in $\operatorname{Perv}(Y)$}

One of the key results of this paper is the geometric proof of the Semisimplicity Theorem for the perverse sheaf ${ }^{m} \mathcal{H}^{0}\left(f_{*} \mathbb{Q}_{X}[n]\right)$. Every perverse sheaf can be written as a finite extension of intersection cohomology complexes. We prove, using induction on a stratification, that all the extensions are trivial. The set-up is as follows. Let $Y$ be an algebraic variety and $s \in \mathbb{N}$ be such that there is a stratification $\mathfrak{Y}$ with $Y=U \amalg S, U=\amalg_{l>s} Y_{l}$ and $S=S_{s}$. Denote by $S \stackrel{\alpha}{\longrightarrow} Y \stackrel{\beta}{\longleftarrow} U$ the corresponding closed and open embeddings. Let $P \in O b(\operatorname{Perv}(Y))$ be $\mathfrak{Y}$-cc.

Consider the truncation triangle

$$
\tau_{\leqslant-s-1} P \rightarrow \tau_{\leqslant-s} P \stackrel{\tau}{\longrightarrow} \tau_{\geqslant-s} P l \stackrel{[1]}{\longrightarrow} .
$$

The conditions of support in 3.6 imply that $\tau_{\leqslant-s-1} \beta^{*} P \simeq \beta^{*} P, P \simeq \tau_{\leqslant-s} P$ and that $\tau_{\geqslant-s} P \simeq$ $\mathcal{H}^{-s}(P)[s]$ is a (shifted) local system supported on $S$. Using the long exact sequences associated with the truncation triangle and with the triangles obtained from it by applying $\alpha^{*}$ and $\alpha^{!}$, one 
checks that the complex $\tau_{\leqslant-s-1} P$ is perverse. We get a short exact sequence in $\operatorname{Perv}(Y)$ :

$$
0 \rightarrow \tau_{\leqslant-s-1} P \rightarrow P \stackrel{\tau}{\longrightarrow} \mathcal{H}^{-s}(P)[s] \rightarrow 0
$$

The deviation of $\tau_{\leqslant-s-1} P$ from being the intermediate extension $\tau_{\leqslant-s-1} \beta_{*} \beta^{*} P \simeq \beta_{! *} \beta^{*} P$ is measured by the map $\tau_{\leqslant-s-1} P \rightarrow \tau_{\leqslant-s-1} \beta_{*} \beta^{*} P$ which arises from truncating the adjunction map $P \rightarrow \beta_{*} \beta^{*} P$.

Since $P \simeq \tau_{\leqslant-s} P$, the adjunction map admits a canonical lifting

$$
l: P \rightarrow \tau_{\leqslant-s} \beta_{*} \beta^{*} P .
$$

We are looking for a condition implying that $P$ is a direct sum of intersection cohomology complexes, or equivalently, that $P \simeq \beta_{!_{*}} \beta^{*} P \oplus \mathcal{H}^{-s}(P)[s]$ holds for every stratum $S_{s}$. In this context the following assumption is natural (cf. Remark 4.1.2).

Assumption 4.1.1. $-\operatorname{dim}_{\mathbb{Q}}\left(\mathcal{H}^{-s}\left(\alpha_{!} \alpha^{!} P\right)\right)_{y}=\operatorname{dim}_{\mathbb{Q}}\left(\mathcal{H}^{-s}\left(\alpha_{*} \alpha^{*} P\right)\right)_{y}, y \in S$.

Remark 4.1.2. - Assumption 4.1.1 is automatically satisfied if, for example, $P$ is a direct sum of intersection cohomology complexes (this is what we are aiming to prove for $\left.{ }^{p} \mathcal{H}^{0}\left(f_{*} \mathbb{Q}_{X}[n]\right)\right)$ or if $P$ is self-dual (in our case, this is automatic by Verdier duality). In the former case, by 3.8, $P$ must be isomorphic to $\beta_{! *} \beta^{*} P \oplus \mathcal{H}^{-s}(P)[s]$ and 4.1 .1 follows from the natural isomorphisms $\alpha^{*} \alpha_{*} \simeq \operatorname{Id} \simeq \alpha^{!} \alpha_{*}$. In the latter case, we apply the duality statement of Lemma 3.5.3.

The triangle $\alpha_{!} \alpha^{!} P \rightarrow P \rightarrow \beta_{*} \beta^{*} P \rightarrow$ gives

$$
\mathcal{H}^{-s-1}(P) \stackrel{a}{\longrightarrow} \mathcal{H}^{-s-1}\left(\beta_{*} \beta^{*} P\right) \rightarrow \mathcal{H}^{-s}(\alpha ! \alpha ! P) \stackrel{\iota}{\longrightarrow} \mathcal{H}^{-s}(P) \stackrel{b}{\longrightarrow} \mathcal{H}^{-s}\left(\beta_{*} \beta^{*} P\right) .
$$

LEMmA 4.1.3 (Splitting Criterion). - Assume 4.1.1. The following are equivalent:

(1) $P \simeq \beta_{! *} \beta^{*} P \oplus \mathcal{H}^{-s}(P)[s]$.

(2) $\iota: \mathcal{H}^{-s}\left(\alpha_{!} \alpha ! P\right) \rightarrow \mathcal{H}^{-s}(P)$ is an isomorphism.

(3) The map $l: P \rightarrow \tau_{\leqslant-s} \beta_{*} \beta^{*} P$ has a lifting $\tilde{l}: P \rightarrow \beta_{! *} \beta^{*} P$.

If (3) holds, then the lifting $\tilde{l}$ is unique and gives the natural isomorphism (cf. (12))

$$
(\tilde{l}, \tau): P \simeq \beta_{!_{*}} P \oplus \mathcal{H}^{-s}(P)[s] .
$$

Proof.-By the characterization of intermediate extensions (cf. 3.8) and the fact that $\alpha^{!} \alpha_{*} \simeq$ Id, (1) implies (2).

Apply the functor $\operatorname{Hom}_{D(Y)}(P,-)$ to the triangle

$$
\tau_{\leqslant-s-1} \beta_{*} \beta^{*} P \rightarrow \tau_{\leqslant-s} \beta_{*} \beta^{*} P \rightarrow \mathcal{H}^{-s}\left(\beta_{*} \beta^{*} P\right)[s] \stackrel{[1]}{\longrightarrow} .
$$

By (11), $\operatorname{Hom}_{D(Y)}^{-1}\left(P, \mathcal{H}^{-s}\left(\beta_{*} \beta^{*} P\right)[s]\right)=\{0\}$. The associated long exact sequence shows that if $\tilde{l}$ exists, then it is unique. Furthermore, $\tilde{l}$ exists if and only if the image of $l$ in $\operatorname{Hom}_{D(Y)}\left(P, \mathcal{H}^{-s}\left(\beta_{*} \beta^{*} P\right)[s]\right)$ is zero. This is equivalent to $b=0$ (cf. (13)) and hence to $\iota$ being surjective hence an isomorphism (cf. 4.1.1). This shows that (2) and (3) are equivalent.

Assume that $\tilde{l}$ exists. We have an exact sequence in the abelian category $\operatorname{Perv}(Y)$ :

$$
0 \rightarrow K \rightarrow P \stackrel{\tilde{l}}{\longrightarrow} \beta_{! *} \beta^{*} P \rightarrow C \rightarrow 0
$$

$4^{\text {e }}$ SÉRIE - TOME $38-2005-\mathrm{N}^{\circ} 5$ 
Since $\tilde{l}$ is an isomorphism over $U$, the complexes $C$ and $K$ are supported on $S$. Since intermediate extensions do not admit quotients supported on $S$, the complex $C=0$. By the conditions of support 3.6, $K \simeq \mathcal{H}^{-s}(K)[s] \simeq \mathcal{H}^{-s}(P)[s]$ is a shifted local system on $S$. The sequence (14) reduces to the triangle

$$
K \rightarrow P \stackrel{\tilde{l}}{\longrightarrow} \beta_{! *} \beta^{*} P \stackrel{[1]}{\longrightarrow}
$$

whose long exact sequence contains

$$
0 \rightarrow \mathcal{H}^{-s-1}(P) \stackrel{\tilde{a}}{\longrightarrow} \mathcal{H}^{-s-1}\left(\beta_{*} \beta^{*} P\right) \stackrel{c}{\longrightarrow} \mathcal{H}^{-s}(K) \rightarrow \mathcal{H}^{-s}(P) \rightarrow 0 .
$$

Since $\iota$ is injective and $\tilde{l}$ is a lifting of $l$, we have that $a=\tilde{a}$ is surjective, so that $c=0$.

Since $\operatorname{Hom}_{D(Y)}\left(\tau_{\leqslant-s-1} \beta_{*} \beta^{*} P, K[1]\right) \simeq \operatorname{Hom}_{S h(Y)}\left(\mathcal{H}^{-s-1}\left(\beta_{*} \beta^{*} P\right), \mathcal{H}^{-s}(P)\right)$, we see that $c=0$ implies that the triangle (15) splits, i.e. that there is some isomorphism $P \simeq \beta_{! *} \beta^{*} P \oplus$ $\mathcal{H}^{-s}(P)[s]$. It follows that $\tilde{l} \oplus \tau$ is an isomorphism on cohomology sheaves, hence an isomorphism. The fact that (3) implies (1) is now trivial.

Remark 4.1.4. - Condition (2) of Lemma 4.1.3 is a local condition. The reader may compare this condition with the splitting criterion along a principal divisor used by M. Saito in [26], Lemme 5.1.4.

\subsection{The perverse filtration}

The perverse truncation functors define increasing filtrations in hypercohomology.

DEFINITION 4.2.1. - Let $K \in O b(D(Y))$ and $j \in \mathbb{Z}$. The perverse filtration on $\mathbb{H}^{j}(Y, K)$ is defined by setting:

$$
\mathbb{H}_{\leqslant i}^{j}(Y, K):=\operatorname{Im}\left\{\mathbb{H}^{j}\left(Y,{ }^{p} \tau_{\leqslant i} K\right) \rightarrow \mathbb{H}^{j}(Y, K)\right\} .
$$

The graded pieces are

$$
\mathbb{H}_{i}^{j}(Y, K):=\mathbb{H}_{\leqslant i}^{j}(Y, K) / \mathbb{H}_{\leqslant i-1}^{j}(Y, K) .
$$

We have canonical maps, $a$ injective, $b$ surjective:

$$
\mathbb{H}^{j}\left(Y,{ }^{p} \mathcal{H}^{i}(K)\right) \stackrel{a}{\longleftarrow} \operatorname{Coker}\left\{\mathbb{H}^{j}\left(Y,{ }^{p} \tau_{\leqslant i-1} K\right) \rightarrow \mathbb{H}^{j}\left(Y,{ }^{p} \tau_{\leqslant i} K\right)\right\} \stackrel{b}{\longrightarrow} \mathbb{H}_{i}^{j}(Y, K)
$$

Given a map $\phi: K \rightarrow K^{\prime}$ in $D(Y)$, the map $\mathbb{H}^{j}(\phi)$ is filtered, but not necessarily strict.

Let $f: X \rightarrow Y$ be a map of algebraic varieties, $n:=\operatorname{dim} X$. There is a canonical isomorphism $H^{n+j}(X) \simeq \mathbb{H}^{j}\left(Y, f_{*} \mathbb{Q}_{X}[n]\right)$.

DEFINITION 4.2.2 (Perverse filtration and cohomology groups). - Let $i, j \in \mathbb{Z}$ and set:

$$
H_{\leqslant i}^{n+j}(X):=\mathbb{H}_{\leqslant i}^{j}\left(Y, f_{*} \mathbb{Q}_{X}[n]\right), \quad H_{i}^{n+j}(X):=H_{\leqslant i}^{n+j}(X) / H_{\leqslant i-1}^{n+j}(X) .
$$

We call the groups $H_{b}^{l}(X)$ the perverse cohomology groups of $X$ (relative to $f$ ).

Note that, in view of the natural equivalence ${ }^{p} \tau_{\leqslant i} \circ[l] \simeq[l] \circ{ }^{p} \tau_{\leqslant i+l}$, we have

$$
H_{\leqslant i}^{n+j}(X) \neq \mathbb{H}_{\leqslant i}^{n+j}\left(Y, f_{*} \mathbb{Q}_{X}\right)=H_{\leqslant i+n}^{n+j}(X) .
$$

The dimensional shift in Definition 4.2.2 is convenient for our purposes. 
Remark 4.2.3. - Let $r: Z^{m} \rightarrow X^{n}, f: X \rightarrow Y$ be maps of algebraic varieties of the indicated dimensions, $g:=f \circ r$. The natural map $f_{*} \mathbb{Q}_{X}[n] \rightarrow g_{*} \mathbb{Q}_{Z}[m][n-m]$ and the rule ${ }^{p}{ }_{\leqslant i} \circ[l] \simeq$ $[l] \circ{ }^{p} \tau_{\leqslant i+l}$ imply that the natural restriction map satisfies (cf. 4.3.9):

$$
r^{*}: H_{\leqslant b}^{l}(X) \rightarrow H_{\leqslant b+n-m}^{l}(Z) .
$$

\section{3. $p$-splitness and some consequences}

In our inductive approach to the results of this paper, the Decomposition Theorem 2.1.1(b) is established rather early (cf. 5.1.3). In this section, we collect some simple consequences of the Decomposition Theorem which are used in the remainder of the proof by induction.

Definition 4.3.1 ( $p$-split). - A complex $K \in D(Y)$ is said to be $p$-split if there is an isomorphism

$$
\phi: K \simeq \bigoplus_{i}^{p} \mathcal{H}^{i}(K)[-i] .
$$

Remark 4.3.2. - We can and shall always consider isomorphisms satisfying the additional condition ${ }^{p} \mathcal{H}^{i}(\varphi)=\operatorname{Id}_{\mathcal{H}} \mathcal{H}^{i}(K)$. Given $\phi$, the map

$$
\varphi:\left(\sum_{i}{ }^{p} \mathcal{H}^{i}\left(\phi^{-1}\right)[-i]\right) \circ \phi: K \rightarrow \bigoplus_{i}{ }^{p} \mathcal{H}^{i}(K)[-i]
$$

is one such isomorphism.

Remark 4.3.3. - If $\phi: K \simeq \bigoplus_{i} P_{i}[-i]$ is an isomorphism with $P_{i} \in \operatorname{Perv}(Y)$ for every $i \in \mathbb{Z}$, then ${ }^{p} \mathcal{H}^{i}(\phi):{ }^{p} \mathcal{H}^{i}(K) \simeq P_{i}$ for every $i \in \mathbb{Z}$ and $K$ is $p$-split.

In the remainder of this section, $f: X \rightarrow Y$ is a proper map of algebraic varieties, $X$ is nonsingular, $n:=\operatorname{dim} X, A$ is an ample line bundle on $Y$ and $L:=f^{*} A$.

Remark 4.3.4. - If $f_{*} \mathbb{Q}_{X}[n]$ is $p$-split, then for $b, j \in \mathbb{Z}$ we have isomorphisms:

$$
\begin{gathered}
H_{\leqslant b}^{n+j}(X) \stackrel{\varphi}{\simeq} \bigoplus_{i \leqslant b} \mathbb{H}^{j-i}\left(Y,{ }^{p} \mathcal{H}^{i}\left(f_{*} \mathbb{Q}_{X}[n]\right)\right), \\
H^{n+j}(X) \stackrel{\varphi}{\simeq} \bigoplus_{b} H_{b}^{n+j}(X) .
\end{gathered}
$$

On the other hand, the maps $a$ and $b$ in (18) are isomorphism and we get a canonical identification:

$$
a \circ b^{-1}: H_{b}^{n+j}(X)=\mathbb{H}^{j-b}\left(Y,{ }^{p} \mathcal{H}^{b}\left(f_{*} \mathbb{Q}_{X}[n]\right)\right) .
$$

The isomorphism induced by $\varphi$ coincides with the one above (cf. 4.3.2).

Let $R: D(Y) \rightarrow D(Y)$ be a functor of triangulated categories, $\nu: R \rightarrow \operatorname{Id}$ (Id $\rightarrow R$, respectively) be a natural transformation of functors of triangulated categories compatible with coproducts and $K \in O b(D(Y))$ be $p$-split. The splitting of $K$ induces a filtration on $\mathbb{H}^{*}(R(K))$. This filtration is independent of the splitting $\varphi$ and need not to coincide with the perverse filtration. The maps $\mathbb{H}^{*}(Y, \nu)$ are strict.

Definition 4.3.5. - Given $R$ and $K p$-split as above, the filtration on $\mathbb{H}^{*}(Y, R(K))$ described above is called the induced filtration.

$4^{\mathrm{e}}$ SÉRIE - TOME $38-2005-\mathrm{N}^{\circ} 5$ 
LEMMA 4.3.6. - Assume that $f_{*} \mathbb{Q}_{X}[n]$ is p-split. Let $U \subseteq Y$ be an euclidean open neighborhood of $y \in Y$ and $U^{\prime}=f^{-1}(U)$. The natural maps

$$
H_{n-l}^{\mathrm{BM}}\left(f^{-1}(y)\right) \rightarrow H_{n-l}^{\mathrm{BM}}\left(U^{\prime}\right) \rightarrow H_{n-l}^{\mathrm{BM}}(X) \simeq H^{n+l}(X) \rightarrow H^{n+l}\left(U^{\prime}\right) \rightarrow H^{n+l}\left(f^{-1}(y)\right)
$$

are strict with respect to the induced filtrations for every $l \in \mathbb{Z}$.

For every $b \in \mathbb{Z}$ :

$$
H_{n-b, \leqslant b}^{\mathrm{BM}}\left(f^{-1}(y)\right)=H_{n-b}^{\mathrm{BM}}\left(f^{-1}(y)\right), \quad H_{\leqslant b-1}^{n+b}\left(f^{-1}(y)\right)=\{0\} .
$$

In particular, the map induced on the graded space

$$
H_{n-b, a}^{\mathrm{BM}}\left(f^{-1}(y)\right) \rightarrow H_{a}^{n+b}\left(f^{-1}(y)\right)
$$

is the zero map for every $a \neq b$.

Proof. - Let $\alpha: y \rightarrow Y$ and $\beta: U \rightarrow Y$ be the embeddings. Note that $f_{*} \omega_{X}[-n] \simeq f_{*} \mathbb{Q}_{X}[n]$ is $p$-split and that the induced filtration on $H^{*}\left(f^{-1}(U)\right)$ coincides with the perverse filtration. The strictness assertions follow from the discussion preceding 4.3 .5 applied to the duality functor and to the two adjunction maps associated with $\alpha$ and $\beta$.

The third statement follows immediately from the second one which in turn follows from the conditions of (co-)support (cf. Remark 3.6.1):

$$
\begin{aligned}
& \mathbb{H}^{t}\left(Y, \alpha ! \alpha^{!} \mathcal{H}^{l}\left(f_{*} \mathbb{Q}_{X}[n][-l]\right)\right)=\{0\}, \quad \forall l>b, \\
& \mathbb{H}^{t}\left(Y, \alpha_{*} \alpha^{* p} \mathcal{H}^{l}\left(f_{*} \mathbb{Q}_{X}[n]\right)[-l]\right)=\{0\}, \quad \forall l<b .
\end{aligned}
$$

Remark 4.3.7. - Theorem 2.1.9 states that the refined intersection product induces isomorphisms $H_{n-b, b}^{\mathrm{BM}}\left(f^{-1}(y)\right) \simeq H_{b}^{n+b}\left(f^{-1}(y)\right)($ cf. 2.1.11).

LEMMA 4.3.8. - Let

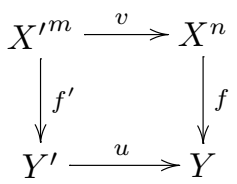

be a Cartesian diagram of maps of algebraic varieties of the indicated dimensions, $f$ proper. Let $\mathfrak{X}$ and $\mathfrak{Y}$ be a stratification for $f$. Assume that $f_{*} \mathbb{Q}_{X}[n]$ is p-split and that either $u$ is smooth, or it is a normally nonsingular inclusion.

Then $f^{\prime}{ }_{*} \mathbb{Q}_{X^{\prime}}[m]$ is p-split, ${ }^{p} \mathcal{H}^{i}\left(f^{\prime}{ }_{*} \mathbb{Q}_{X^{\prime}}[m]\right) \simeq u^{* p} \mathcal{H}^{i}\left(f_{*} \mathbb{Q}_{X}[n]\right)[m-n]$, for every $i \in \mathbb{Z}$ and the natural map $v^{*}: H^{k}(X) \rightarrow H^{k}\left(X^{\prime}\right)$ is compatible with the direct sum decomposition in perverse cohomology groups and it is strict.

Let $y^{\prime} \in Y^{\prime}$ and $y=u\left(y^{\prime}\right)$. Then the filtrations induced by $f$ and $f^{\prime}$ on the (co)-homology of $f^{-1}(y)=f^{\prime-1}\left(y^{\prime}\right)$ coincide.

Proof. - Let $\varphi: \bigoplus_{i}{ }^{p} \mathcal{H}^{i}\left(f_{*} \mathbb{Q}_{X}[n]\right)[-i] \simeq f_{*} \mathbb{Q}_{X}[n]$ be a splitting. By base change,

$$
u^{*} \varphi: u^{*}\left(\bigoplus_{i} \mathcal{H}^{i}\left(f_{*} \mathbb{Q}_{X}[n]\right)[-i][m-n]\right) \stackrel{\simeq}{\longrightarrow} u^{*} f_{*} \mathbb{Q}_{X}[n][m-n] \simeq f^{\prime}{ }_{*} \mathbb{Q}_{X^{\prime}}[m] .
$$

By 3.7 and by Remark 3.5.1, respectively, the hypotheses imply that $u^{*}[m-n]$ is $t$-exact so that the left-hand side is a direct sum of shifted perverse sheaves. The first two statements follow (cf. 4.3.3). 
The pull-back map $v^{*}$ on cohomology stems from adjunction and base change, $f_{*} \mathbb{Q}_{X} \rightarrow$ $f_{*} v_{*} v^{*} \mathbb{Q}_{X} \simeq u_{*} f_{*}^{\prime} v^{*} \mathbb{Q}_{X} \simeq u_{*} u^{*} f_{*} \mathbb{Q}_{X}$, and preserves direct sum decompositions so that $v^{*}$ is strict.

The last statement follows from (20).

Remark 4.3.9. - The same conclusions hold if $u=u^{\prime \prime} \circ u^{\prime}$, with $u^{\prime}$ smooth and $u^{\prime \prime}$ a $\mathfrak{Y}$-transverse embedding into $Y$. Note how the last statement improves on Remark 4.2.3.

\subsection{The cup product with a line bundle}

Let $\mu$ be a line bundle on $X$ and denote by the same symbol its first Chern class $\mu \in H^{2}(X) \simeq$ $\operatorname{Hom}_{D(X)}\left(\mathbb{Q}_{X}, \mathbb{Q}_{X}[2]\right)$. For every $K \in O b(D(X))$, the isomorphism $K \simeq K \otimes \mathbb{I} \mathbb{Q}_{X}$ defines a $\operatorname{map} \mu: K \rightarrow K[2]$.

Remark 4.4.1. - Suppose $s \in \Gamma(X, \mu)$ is a section whose zero locus defines a normally nonsingular codimension one inclusion $i:\{s=0\} \rightarrow X$; see 3.5. By Lemma 3.5.4 the map $\mu: K \rightarrow K[2]$ can be described geometrically as the composition:

$$
K \rightarrow i_{*} i^{*} K \simeq i_{!} i^{!} K[2] \rightarrow K[2] .
$$

If $K=\mathbb{Q}_{X}$, then $\mu$ is the cohomology class associated with the normally nonsingular inclusion $i$ and we find one of the classical definitions of first Chern class.

The resulting map $\mathbb{H}^{i}(X, \mu): \mathbb{H}^{i}(X, K) \rightarrow \mathbb{H}^{i+2}(X, K)$ is the cup product with $\mu$.

By functoriality, we get maps

$$
\begin{aligned}
f_{*} \mu: f_{*} K & \rightarrow f_{*} K[2], \\
{ }_{\tau_{\leqslant i} f_{*} \mu:}{ }^{p} \tau_{\leqslant i} f_{*} K & \rightarrow\left({ }^{p} \tau_{\leqslant i+2} f_{*} K\right)[2], \\
{ }^{p} \mathcal{H}^{i}\left(f_{*} \mu\right):{ }^{p} \mathcal{H}^{i}\left(f_{*} K\right) & \rightarrow{ }^{p} \mathcal{H}^{i+2}\left(f_{*} K\right) .
\end{aligned}
$$

Applying the functor $\mathbb{H}^{*}(Y,-)$, we obtain the cup product with $\mu$

$$
\mathbb{H}(X, \mu)=\mathbb{H}\left(Y, f_{*} \mu\right): \mathbb{H}_{\leqslant a}^{*}(X, K) \rightarrow \mathbb{H}_{\leqslant a+2}^{*+2}(X, K)
$$

which is filtered in an obvious sense and defines a cup product map on the graded objects, still denoted

$$
\mu: H_{a}^{*}(X, K) \rightarrow H_{a+2}^{*+2}(X, K) .
$$

Let $\varphi: K \simeq \bigoplus_{i}{ }^{p} \mathcal{H}^{i}(K)[-i]$ be a $p$-splitting as in 4.3.2. We have

$$
\varphi[2] \circ f_{*} \mu \circ \varphi^{-1}=: \tilde{\mu}=\sum_{i j} \tilde{\mu}_{i j}: \bigoplus_{i}{ }^{p} \mathcal{H}^{i}(K)[-i] \rightarrow \bigoplus_{j}{ }^{p} \mathcal{H}^{j}(K)[-j][2] .
$$

By the choice of $\varphi, \tilde{\mu}_{i, i+2}={ }^{p} \mathcal{H}^{i}\left(f_{*} \mu\right)$. By (11), $\tilde{\mu}_{i j}=0$ for $j>i+2$. In general, $\tilde{\mu}_{i j} \neq 0$ for $j<i+2$; e.g. if $f=\operatorname{Id}_{X}$, then $\mu=\tilde{\mu}=\tilde{\mu}_{00}$. It is immediate to verify the following important compatibility:

$4^{\mathrm{e}}$ SÉRIE - TOME $38-2005-\mathrm{N}^{\circ} 5$ 
LEMMA 4.4.2. - Let $f_{*} K$ be p-split (cf. 4.3.2), then the isomorphism $\mathbb{H}^{*-i}\left(Y,{ }^{p} \mathcal{H}^{i}\left(f_{*} K\right)\right) \simeq$ $\mathbb{H}_{i}^{*}(X, K)$ of 4.3.4 is compatible with $\mu$, i.e. the following diagram is commutative

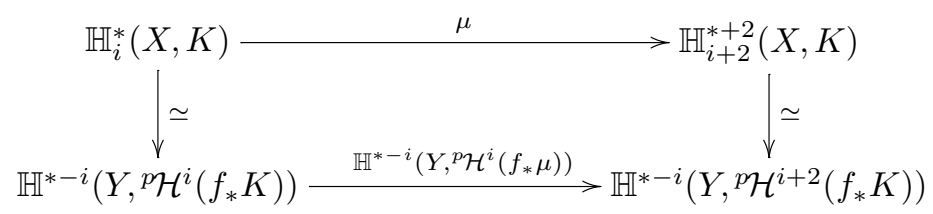

Remark 4.4.3. - Let $\nu$ be a line bundle on $Y$, denote the first Chern class in $H^{2}(Y)$ by $\nu$ and let $\mu=f^{*} \nu$. Then $f_{*} \mu=f_{*} f^{*} \nu: f_{*} K \rightarrow f_{*} K[2]$ coincides with $\nu$, as it can be seen by considering a section $s \in \Gamma(Y, \nu)$ such that $\{s=0\} \rightarrow Y$ and $f^{-1}(\{s=0\})=\left\{f^{*} s=0\right\} \rightarrow X$ are normally nonsingular. This also follows from the Change of Coefficients Formula in 3.3. In particular, if $f_{*} K \simeq \bigoplus_{i}{ }^{p} \mathcal{H}^{i}\left(f_{*} K\right)[-i]$ is $p$-split, then $f_{*} \mu=f_{*} f^{*} \nu=\nu$ is a direct sum map sending ${ }^{p} \mathcal{H}^{i}\left(f_{*} K\right)$ to ${ }^{p} \mathcal{H}^{i}\left(f_{*} K\right)[2]$. In this case, ${ }^{p} \mathcal{H}^{i}\left(\left(f_{*} \mu\right)^{r}\right):{ }^{p} \mathcal{H}^{i}\left(f_{*} K\right) \rightarrow{ }^{p} \mathcal{H}^{i+2 r}\left(f_{*} K\right)$ is the zero map, for every $r>0$ and the cup product map with a pull-back is filtered strict

$$
f^{*} \nu: H_{i}^{*}(X) \rightarrow H_{i}^{*+2}(X)
$$

with a compatibility analogous to the one in Lemma 4.4.2.

THEOREM 4.4.4. - Suppose that

$$
{ }^{p} \mathcal{H}^{-i}\left(f_{*}(\mu)^{i}\right):{ }^{p} \mathcal{H}^{-i}\left(f_{*} \mathbb{Q}_{X}[n]\right) \simeq{ }^{p} \mathcal{H}^{i}\left(f_{*} \mathbb{Q}_{X}[n]\right), \quad \forall i \geqslant 0
$$

Then:

(a) $f_{*} \mathbb{Q}_{X}[n]$ is p-split.

(b) Let $i \geqslant 0, k \leqslant l$ and define

$$
\begin{aligned}
\mathcal{P}_{\mu}^{-i} & :=\operatorname{Ker}\left\{{ }^{p} \mathcal{H}^{-i}\left(f_{*}(\mu)^{i+1}\right):{ }^{p} \mathcal{H}^{-i}\left(f_{*} \mathbb{Q}_{X}[n]\right) \rightarrow{ }^{p} \mathcal{H}^{i+2}\left(f_{*} \mathbb{Q}_{X}[n]\right)\right\}, \\
\mu^{k} \mathcal{P}_{\mu}^{-l} & :=\operatorname{Im}\left\{{ }^{p} \mathcal{H}^{-l}\left(f_{*} \mu^{k}\right): \mathcal{P}_{\mu}^{-l} \rightarrow{ }^{p} \mathcal{H}^{-l+2 k}\left(f_{*} \mathbb{Q}_{X}[n]\right)\right\} .
\end{aligned}
$$

(Note that the map above is a split monomorphism). There is a direct sum decomposition:

$$
{ }^{p} \mathcal{H}^{-i}\left(f_{*} \mathbb{Q}_{X}[n]\right) \simeq \bigoplus_{k \geqslant 0} \mu^{k} \mathcal{P}_{\mu}^{-i-2 k}, \quad{ }^{p} \mathcal{H}^{i}\left(f_{*} \mathbb{Q}_{X}[n]\right) \simeq \bigoplus_{k \geqslant 0} \mu^{i+k} \mathcal{P}_{\mu}^{-i-2 k}
$$

(c) Let $i \geqslant 0, j \in \mathbb{Z}$. The isomorphisms

$$
H_{-i}^{n+j}(X) \simeq \mathbb{H}^{j}\left(Y,{ }^{p} \mathcal{H}^{-i}\left(f_{*} \mathbb{Q}_{X}[n]\right)[i]\right) \simeq \bigoplus_{k \geqslant 0} \mathbb{H}^{j+i}\left(Y, \mu^{k} \mathcal{P}_{\mu}^{-i-2 k}\right)
$$

identify:

(1) $\operatorname{Ker} \mu^{i+1} \subseteq H_{-i}^{n+j}(X)$ with the summand $\mathbb{H}^{j+i}\left(Y, \mathcal{P}_{\mu}^{-i}\right)$ and

(2) the image of the injection $\mu^{k}: H_{-i-2 k}^{n+j-2 k}(X) \rightarrow H_{-i}^{n+j}(X)$ restricted to $\mathbb{H}^{j+i}\left(Y, \mathcal{P}_{\mu}^{-i-2 k}\right)$, with the summand $\mathbb{H}^{j+i}\left(Y, \mu^{k} \mathcal{P}_{\mu}^{-i-2 k}\right)$.

Proof. - For (a) and (b) see [9]. The rest follows from the constructions and Lemma 4.4.2.

Remark 4.4.5. - Given $\varphi: f_{*} \mathbb{Q}_{X}[n] \simeq \bigoplus_{i}{ }^{p} \mathcal{H}^{i}\left(f_{*} \mathbb{Q}_{X}[n]\right)[-i], \quad$ the space $\left.\varphi^{-1} \mathbb{H}^{-i}\left(Y, \mathcal{P}_{\eta}^{-i}[i]\right)\right) \subseteq H_{\leqslant-i}^{n-i}(X)$ is not contained in $\operatorname{Ker} \eta^{i+1}$, i.e. the space of classical 
primitive classes. What is true is that $\mathbb{H}^{l}\left(Y, \mathcal{P}_{\eta}^{-i}[i]\right)$ is the kernel of $\eta^{i+1}: H_{-i}^{n+l}(X) \rightarrow$ $H_{i+2}^{n+l+2 i+2}(X)$.

Remark 4.4.6. - In the sequel of this paper, for simplicity of notation, we shall denote by the same symbol all the cup product morphisms, without indicating which functor has been applied; e.g. we will use $\mu$ instead of $f_{*} \mu,{ }^{p} \tau_{\leqslant i} f_{*} \mu,{ }^{p} \mathcal{H}^{i}\left(f_{*} \mu\right)$ etc.

\subsection{Weight filtrations}

For the notions introduced in this section see [13] and [31]. An increasing filtration $W$ on a finite dimensional vector space $H$ is a collection of subspaces $W_{i} \subseteq W$ such that $W_{i-1} \subseteq W_{i}$ for every $i \in \mathbb{Z}$. The associated graded spaces are $G r_{i}^{W} H:=W_{i} / W_{i-1}$ The pair $(H, W)$ is called a filtered space.

A splitting of $(H, W)$ is an isomorphism $H \simeq \bigoplus G r_{i}^{W} H$.

Let $j \in \mathbb{Z}$. The shifted filtration $W[j]$ is defined by setting $W[j]_{i}:=W_{j+i}$.

A filtered map $\varphi:(H, W) \rightarrow\left(H^{\prime}, W^{\prime}\right)$ is a linear map $\varphi: H \rightarrow H^{\prime}$ such that $\varphi\left(W_{i}\right) \subseteq W_{i}^{\prime}$. It induces linear maps $\operatorname{Gr} \varphi: G r_{i}^{W} H \rightarrow G r_{i}^{W^{\prime}} H^{\prime}$ which we simply denote by $\varphi$.

A filtered map $\varphi$ is called strict if $\varphi\left(W_{i}\right)=\varphi(H) \cap W_{i}^{\prime}$.

Given a space $H^{\prime}$ which is either a subspace or a quotient of $H$, one easily defines an induced filtration $W\left(H^{\prime}\right)$ so that the maps in sight are filtered. In particular, given two filtrations $W$ and $W^{\prime}$ on $H$, the associated graded spaces $G r_{i}^{W} H:=W_{i} / W_{i-1}$ are naturally filtered by $W^{\prime}$.

Given a finite dimensional vector space $H$ and a nilpotent endomorphism $N$, there is a unique filtration $W$ with the properties that (i) $N W_{i} \subseteq W_{i-2}$ and, denoting again by $N$ the induced map on graded spaces, (ii) $N^{i}: G r_{i}^{W} H \simeq G r_{-i}^{W} H$, for every $i \geqslant 0$ (cf. [13]).

Set $N^{i}=0, P^{-i}=0$ if $i<0, P^{-i}=\operatorname{Ker} N^{i+1} \subseteq G r_{i}^{W}$, if $i \geqslant 0$. There is the Lefschetz decomposition

$$
G r_{i}^{W}=\bigoplus_{l \in \mathbb{Z}} N^{-i+l} P^{i-2 l}, \quad i \in \mathbb{Z}
$$

This unique filtration is called the weight filtration of $N$ and is given by the "convolution formula"

$$
W_{k}=\sum_{i+j=k} \operatorname{Ker} N^{i+1} \cap \operatorname{Im} N^{-j} .
$$

We denote the weight filtration of $N$ by $W^{N}$ and the graded spaces by $G r_{i}^{N}$.

Let $S$ be a nondegenerate bilinear form on $H$ which is either symmetric or skew-symmetric and satisfies

$$
S(N a, b)+S(a, N b)=0 .
$$

When (24) holds, one says that $N$ is an infinitesimal automorphism of $(H, S)$. In this case, the weight filtration is self-dual, i.e.

$$
\left(W_{i}^{N}\right)^{\perp}=W_{-i-1}^{N}, \quad i \in \mathbb{Z}
$$

and $S$ descends to nondegenerate forms on $G r_{i}^{N} H$ for every $i \in \mathbb{Z}$. More precisely,

$$
S_{i}^{N}([a],[b]):=S\left(a, N^{i} b\right), \quad \text { for } i \geqslant 0
$$

and one requires that $N^{i}: G r_{i}^{N} H \simeq G r_{-i}^{N} H$ is an isometry for every $i \geqslant 0$. This is achieved as follows: if $[a],[b] \in G r_{-i}^{N} H$, we have $[a]=N^{i}\left[a^{\prime}\right]$ and $[b]=N^{i}\left[b^{\prime}\right]$ for uniquely determined $4^{\mathrm{e}}$ SÉRIE - TOME $38-2005-\mathrm{N}^{\circ} 5$ 
$\left[a^{\prime}\right],\left[b^{\prime}\right] \in G r_{i}^{N} H$; set

$$
S_{-i}^{N}([a],[b]):=S_{i}^{N}\left(\left[a^{\prime}\right], N^{i}\left[b^{\prime}\right]\right), \quad i>0 .
$$

The Lefschetz decomposition (22) is $S_{i}^{N}$-orthogonal for every $i \in \mathbb{Z}$.

Let $(H, W)$ be a filtered space. For every $i \in \mathbb{Z}$, a nilpotent endomorphism $N$ of $(H, W)$ descends to a nilpotent map $G r_{i} N: G r_{i}^{W} H \rightarrow G r_{i}^{W} H$ and yields the weight filtration $W^{G r_{i} N}$ on $G r_{i}^{W} H$.

Let $k \in \mathbb{Z}$ be fixed. There is at most one filtration $W^{\prime}$ of $H$, called the weight- $k$ filtration of $N$ relative to $W$, such that (i) $N W_{i}^{\prime} \subseteq W_{i-2}^{\prime}$ and (ii) $W^{\prime}\left(G r_{k}^{W} H\right)=W^{G r_{k} N}$. See [31].

Let $(H, S)$ be as above, $N$ and $M$ be commuting nilpotent infinitesimal automorphisms of $(H, S)$. By the convolution formula (23) for $W^{N}$, one has $M W_{j}^{N} \subseteq W_{j}^{N}$. For ease of notation we denote the map induced by $M$ on $G r_{j}^{N} H$ simply by $M$.

Assume that $W^{M}[j]$ is the weight- $j$ filtration of $M$ relative to $W^{N}$ on $H$ for every $j \in \mathbb{Z}$. In particular, this means that

$$
M^{i}: G r_{j+i}^{M} G r_{j}^{N} H \simeq G r_{j-i}^{M} G r_{j}^{N} H, \quad i \geqslant 0 .
$$

Set $P_{-i}^{-j}=\operatorname{Ker} M^{i+1} \cap \operatorname{Ker} N^{j+1} \subseteq G r_{j+i}^{M} G r_{j}^{N} H$ if $i, j \geqslant 0$ and zero otherwise. We have the double Lefschetz decomposition

$$
G r_{j+i}^{M} G r_{j}^{N} H=\bigoplus_{l, m \in \mathbb{Z}} M^{-i+l} N^{-j+m} P_{i-2 l}^{j-2 m}, \quad i, j \in \mathbb{Z} .
$$

The nondegenerate forms $S_{j}^{N}$ descend to nondegenerate forms $S_{i j}^{M N}$ on $G r_{j+i}^{M} G r_{j}^{N} H$. For $i, j \geqslant 0$ we have

$$
S_{i j}^{M N}([a],[b])=S\left(a, M^{i} N^{j} b\right),
$$

where $a, b \in W_{j+i}^{M} H \cap W_{j}^{N} H$ are representatives of $[a],[b] \in G r_{j+i}^{M} G r_{j}^{N} H$. For the remaining values of $i$ and $j, S_{i j}^{M N}$ is defined by imposing that (28) is an isometry (cf. (27)).

The decomposition (29) is $S_{i j}^{N M}$-orthogonal.

Remark 4.5.1. - Let $n \in \mathbb{Z}$ be fixed and assume that the spaces $G r_{j+i}^{M} G r_{j}^{N} H$ are pure Hodge structures of weight $(n-i-j)$ and that the induced maps

$$
\begin{aligned}
& N: G r_{j+i}^{M} G r_{j}^{N} H \rightarrow G r_{j-2+i}^{M} G r_{j-2}^{N} H, \\
& M: G r_{j+i}^{M} G r_{j}^{N} H \rightarrow G r_{j+i-2}^{M} G r_{j}^{N} H
\end{aligned}
$$

are of pure type $(1,1)$. Then (29) is a direct sum of pure Hodge substructures.

Remark 4.5.2. - If in addition the form $(-1)^{n-i-j} S_{i j}^{M N}$ is a polarization of $P_{-i}^{-j}$ for every pair of indices $(i, j) \neq(0,0)$ such that $i, j \geqslant 0$, then $(-1)^{i+j-m-l-1} S_{i j}^{M N}$ is a polarization of the summands $M^{-i+l} N^{-j+m} P_{i-2 l}^{j-2 m}$ in (29) except, possibly, for $P_{0}^{0}$. In this case, we simply say that the forms $S_{i j}^{M N}$ polarize the summand spaces in question up to sign.

\subsection{Filtrations on $H^{*}(X)=\bigoplus_{j} H^{j}(X)$}

Let $f: X \rightarrow Y$ be a map of projective varieties, $X$ nonsingular, $n=\operatorname{dim} X, \eta$ be an ample line bundle on $X, A$ be an ample line bundle on $Y$ and $L=f^{*} A$. Let

$$
H^{*}(X):=\bigoplus_{l} H^{l}(X)
$$


and define the twisted Poincaré form by setting:

$$
S\left(\sum \alpha_{l}, \sum \beta_{l}\right):=\sum_{l}(-1)^{l(l-1) / 2} \int_{X} \alpha_{l} \wedge \beta_{2 n-l} .
$$

Note that the bilinear form $S$ on $H^{*}(X)$ is $(-1)^{n}$-symmetric, that $\eta$ and $L$ act via cup product as nilpotent, commuting operators on $H^{*}(X)$ with $\eta^{n+1}=L^{n+1}=0$ and that $\eta$ and $L$ are infinitesimal automorphisms of $\left(H^{*}(X), S\right)$ (cf. 4.5).

The line bundles $\eta$ and $L$ act via cup product on the cohomology of $X$ in a nilpotent fashion and induce the weight filtrations $W^{\eta}$ and $W^{L}$ on $H^{*}(X)$.

By the classical Hard Lefschetz Theorem 3.1.2 the weight filtration $W^{\eta}$ for $\eta$ is the filtration by degree $W^{\text {deg: }}$

$$
W_{i}^{\eta}=W^{\mathrm{deg}}:=\bigoplus_{l \geqslant n-i} H^{l}(X) .
$$

We also consider the total filtration on $H^{*}(X)$ :

$$
W_{i}^{\text {tot }}:=\bigoplus_{b \in \mathbb{Z}} H_{\leqslant b+i}^{n+b}(X)
$$

Clearly,

$$
G r_{j+i}^{\eta} G r_{j}^{\text {tot }} H^{*}(X)=H_{-i}^{n-i-j}(X), \quad i, j \in \mathbb{Z}
$$

and (21) implies:

$$
\eta W_{i}^{\mathrm{deg}} \subseteq W_{i-2}^{\mathrm{deg}}, \quad \eta W_{j}^{\mathrm{tot}} \subseteq W_{j}^{\text {tot }} .
$$

One of the main results of this paper is that $W^{L}=W^{\text {tot }}$, i.e. that, roughly speaking, the perverse filtration coincides with the weight filtration induced by $L=f^{*} A$ (cf. 5.2.4).

\subsection{The Universal Hyperplane section and the defect of semismallness}

The universal hyperplane section plays a very important role in our inductive proof of the Relative Hard Lefschetz Theorem. In this section we prove that the defect of semismallness $r(f)$ of a map decreases when taking a map naturally associated with the universal hyperplane section. We also prove Weak Lefschetz-type results.

Let $X \subseteq \mathbb{P}$ be an embedded quasi-projective variety and consider the universal hyperplane section diagram

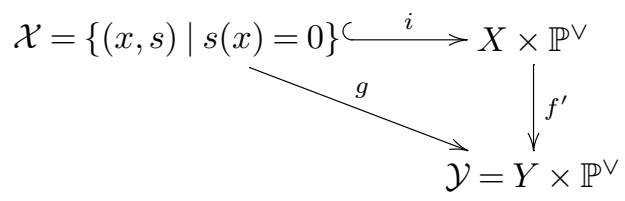

Let $j:\left(X \times \mathbb{P}^{\vee}\right) \backslash \mathcal{X} \rightarrow X \times \mathbb{P}^{\vee}$ be the open embedding. The morphism $u:=f^{\prime} \circ j$ is affine.

In the special case $X=\mathbb{P}$ with $f=\operatorname{Id}_{\mathbb{P}}$ we get $\mathcal{P}:=\{(p, s) \mid s(p)=0\} \subseteq \mathbb{P} \times \mathbb{P}^{\vee}$ which is nonsingular of codimension one and for which the natural projection $\mathcal{P} \rightarrow \mathbb{P}$ is smooth.

A stratification $\mathfrak{X}$ of $X$ with strata $S_{l}$ induces a stratification on $X \times \mathbb{P}^{\vee}$ with strata $S_{l} \times \mathbb{P}^{\vee}$. The following is elementary and left to the reader. See 3.8 and Lemma 3.5.4(b).

$4^{\text {e }}$ SÉRIE - TOME $38-2005-\mathrm{N}^{\circ} 5$ 
Proposition 4.7.1. - The embedding $i: \mathcal{X} \rightarrow X \times \mathbb{P}^{\vee}$ is transversal with respect to the stratification induced by any stratification of $X$, i.e. the intersection $\mathcal{P} \cap\left(X \times \mathbb{P}^{\vee}\right)=\mathcal{X}$ is transversal along every stratum $S_{l} \times \mathbb{P}^{\vee}$ of $X \times \mathbb{P}^{\vee}$. In particular,

$$
I C_{\mathcal{X}} \simeq i^{*} I C_{X \times \mathbb{P} \vee}[-1] \simeq i^{*} p^{\prime *} I C_{X}[d][-1] .
$$

Let $f: X \rightarrow Y$ be as in 2.1. We recall the definition of the defect of semismallness of the map $f$. It plays a crucial role in Goresky-MacPherson's version of the Weak Lefschetz Theorem in [17]. Set $Y^{i}=\left\{y \in Y \mid \operatorname{dim} f^{-1}(y)=i\right\}$.

DEFINITION 4.7.2. - The defect of semismallness of the map $f$ is the integer

$$
r=r(f):=\max _{i \mid Y^{i} \neq \emptyset}\left\{2 i+\operatorname{dim} Y^{i}-\operatorname{dim} X\right\} .
$$

Note that $r(f) \geqslant 0$. If $r(f)=0$, then we say $f$ is semismall. Note that this implies that $f$ is generically finite. If $r(f)=0$ and the maximum is realized only for $i=0$, then we say that $f$ is small.

Remark 4.7.3. - Let $f$ be as in 2.1. If $f$ is semismall, then $f_{*} \mathbb{Q}_{X}[n] \simeq{ }^{p} \mathcal{H}^{0}\left(f_{*} \mathbb{Q}_{X}[n]\right)$ and Theorems 2.1.1(a), (b) hold trivially. In fact, $r(f)=0$ implies that $f_{*} \mathbb{Q}_{X}[n]$ satisfies the conditions of support of Remark 3.6.1 (cf. [3]). The conditions of co-support are automatic since $f_{*} \mathbb{Q}_{X}[n]$ is self-dual.

The geometric quantity $r(f)$ plays a crucial role in our proof by induction. The key point is that if it is not zero, then it decreases by taking hyperplane sections.

LEMMA 4.7.4 $(r(f)$ goes down). -

(a) If $r(f)>0$, then $r(g)<r(f)$.

(b) If $r(f)=0$, then $g$ is small.

Proof. - For $s \in \mathbb{P}^{\vee}$, let $X_{s}:=\{x \in X \mid s(x)=0\}$ be the corresponding hyperplane section. If $(y, s) \in \mathcal{Y}$, then the projection $p: \mathcal{X} \rightarrow X$ identifies $g^{-1}(y, s)$ with $f^{-1}(y) \cap X_{s}$. Set

$$
\mathcal{Y}^{i \prime}=\left\{(y, s): \operatorname{dim} f^{-1}(y)=i=\operatorname{dim} f^{-1}(y) \cap X_{s}\right\} .
$$

The point $(y, s) \in \mathcal{Y}^{i \prime}$ if and only if $X_{s}$ contains a top dimensional component of $f^{-1}(y)$. It is a closed algebraic subset of $\mathcal{Y}^{i}$. Set

$$
\mathcal{Y}^{i \prime \prime}=\left\{(y, s) \mid \operatorname{dim} f^{-1}(y)=i+1 \text { and } \operatorname{dim} f^{-1}(y) \cap X_{s}=i\right\} .
$$

It is an open algebraic subset of $\mathcal{Y}^{i}$. We have that

$$
\mathcal{Y}^{i}=\mathcal{Y}^{i \prime} \amalg \mathcal{Y}^{i \prime \prime} .
$$

Since the set of hyperplanes in a projective space containing a given irreducible subvariety of dimension $d$ is a linear space of codimension at least $d+1$, the definition of $r(f)$ implies that $\operatorname{dim} \mathcal{Y}^{i \prime} \leqslant \operatorname{dim} Y^{i}+\operatorname{dim} \mathbb{P}^{\vee}-(i+1) \leqslant r(f)-2 i+\operatorname{dim} X+\operatorname{dim} \mathbb{P}^{\vee}-(i+1)=$ $r(f)-3 i+\operatorname{dim} \mathcal{X}$. It follows that

$$
2 i+\operatorname{dim} \mathcal{Y}^{i \prime}-\operatorname{dim} \mathcal{X} \leqslant r(f)-i, \quad \forall i \geqslant 0 .
$$


Since the general hyperplane section does not contain any irreducible component of $f^{-1}(y)$, we have that $\operatorname{dim} \mathcal{Y}^{i \prime \prime}=\operatorname{dim} Y^{i+1}+\operatorname{dim} \mathbb{P}^{\vee} \leqslant r(f)+-2(i+1)+\operatorname{dim} X+\operatorname{dim} \mathbb{P}^{\vee}=$ $r(f)-1-2 i+\operatorname{dim} \mathcal{X}$. It follows that

$$
2 i+\operatorname{dim} \mathcal{Y}^{i \prime \prime}-\operatorname{dim} \mathcal{X} \leqslant r(f)-1, \quad \forall i \geqslant 0 .
$$

Suppose that either $Y^{0}$ is empty, or $\operatorname{dim} Y^{0}-\operatorname{dim} X<r(f)$. Then the first inequality above is strict for $i=0$. Combining it with the second inequality, we get that $r(g) \leqslant r(f)-1$.

Suppose that $Y^{0}$ is not empty and that $\operatorname{dim} Y^{0}-\operatorname{dim} X=r(f)$. Then $r(f)=0$. The two inequalities above give $r(g) \leqslant r(f)$, hence $r(g)=0$. Moreover, $\operatorname{dim} \mathcal{Y}^{i}-2 i+\operatorname{dim} \mathcal{X}<0, \forall i>0$ so that $g$ is small.

A similar argument, based on "Hironaka's principle of counting constants", as explained in [29], proves the following proposition, left to the reader:

PROpOSITION 4.7.5. - Let $X$ be nonsingular and $\eta$ be an ample line bundle on $X$. There exists $m_{0} \gg 0$ such that for every $m \geqslant m_{0}$, having denoted by $X^{k}$ the transversal intersection of $k$ general hyperplane sections in $|m \eta|, k \geqslant 1$ and by $f_{k}: X^{k} \rightarrow Y$ the resulting morphism, we have:

(a) If $r(f) \geqslant k$, then $r\left(f_{k}\right) \leqslant r(f)-k$.

(b) If $r(f)=0$, then $f_{1}: X^{1} \rightarrow Y$ is small.

The left $t$-exactness of affine maps has important implications for the topology of algebraic varieties.

LEMMA 4.7.6 (Left $t$-exactness and Weak Lefschetz). - Let

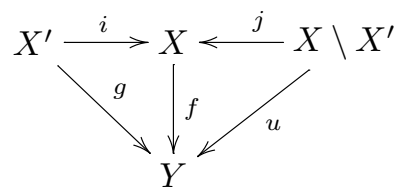

be a commutative diagram of algebraic varieties with $i$ a closed embedding, $f$ proper, $u$ affine and let $P \in \operatorname{Perv}(X)$. Then

(i) the natural map ${ }^{\eta} \mathcal{H}^{l}\left(f_{*} P\right) \rightarrow{ }^{\eta} \mathcal{H}^{l}\left(g_{*} i^{*} P\right)$ is iso for $l \leqslant-2$ and mono for $l=-1$;

(ii) the natural map ${ }^{p} \mathcal{H}^{l}\left(g_{*} i^{!} P\right) \rightarrow{ }^{p} \mathcal{H}^{l}\left(f_{*} P\right)$ is iso for $l \geqslant 2$ and epi for $l=1$.

Proof. - By applying $f_{*} \simeq f_{!}$to the triangle $j_{!} j^{!} P \rightarrow P \rightarrow i_{*} i^{*} P \rightarrow$ and by using the isomorphisms $u_{!} \simeq f_{!} j_{!}, j^{!} \simeq j^{*}, f_{*} i_{*} \simeq g_{*}$ one gets the triangle $u_{!} j^{*} P \rightarrow f_{*} P \rightarrow g_{*} i^{*} P \rightarrow$. Since $j^{*} P \in \operatorname{Perv}\left(X \backslash X^{\prime}\right)$ and $u$ ! is left $t$-exact, (i) follows by taking the long exact sequence of perverse cohomology on $Y$.

(ii) is obtained by first applying (i) to $\mathcal{D}(P) \in \operatorname{Perv}(X)$ and then by applying the rules $\mathcal{D}^{p} \mathcal{H}^{l}(-) \simeq{ }^{p} \mathcal{H}^{-l}(\mathcal{D}(-)), \mathcal{D} f_{*} \mathcal{D} \simeq f_{!} \simeq f_{*}$ and $\mathcal{D} g_{*} i^{*} \mathcal{D} \simeq g_{!} i i^{!} \mathcal{D} \mathcal{D} \simeq g_{*} i^{!}$.

We shall need the following immediate consequence of Lemma 4.7.6.

Proposition 4.7.7. - Let $Y$ be a projective variety, $i: Y_{1} \rightarrow Y$ be a hyperplane section and $P \in \operatorname{Perv}(Y)$. Then the natural maps

$$
i^{*}: \mathbb{H}^{j}(Y, P) \rightarrow \mathbb{H}^{j}\left(Y_{1}, i^{*} P\right), \quad i_{*}: \mathbb{H}^{l}\left(Y_{1}, i^{!} P\right) \rightarrow \mathbb{H}^{l}(Y, P)
$$

are isomorphisms for $j \leqslant-2$ and $l \geqslant 2$, injective for $j=-1$ and surjective for $l=1$.

$4^{\mathrm{e}}$ SÉRIE - TOME $38-2005-\mathrm{N}^{\circ} 5$ 
Proof. - Apply Lemma 4.7.6 and take hypercohomology.

Consider the universal hyperplane section in 4.7. There is the commutative diagram

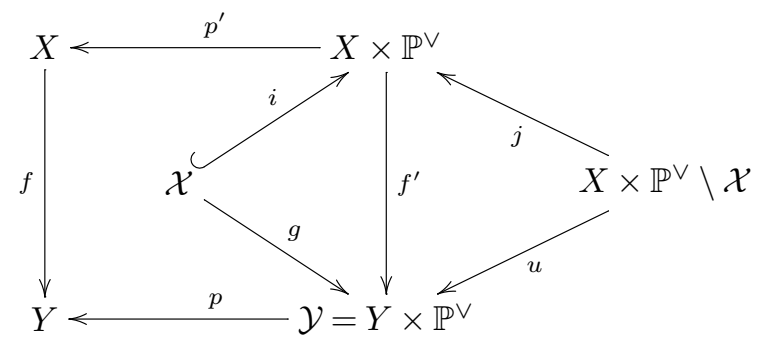

where $u:=f^{\prime} \circ j$ is an affine morphism.

Proposition 4.7.8 (The Relative Weak Lefschetz Theorem). - Let $K \in \operatorname{Perv}(X), K^{\prime}:=$ $p^{\prime *} K[d], M:=i^{*} K^{\prime}[-1]$. Then

(i) $p^{*} \mathcal{H}^{l}\left(f_{*} K\right)[d] \rightarrow \mathcal{H}^{l+1}\left(g_{*} M\right)$ is iso for $l \leqslant-2$ and mono for $l=-1$;

(ii) ${ }^{p} \mathcal{H}^{l-1}\left(g_{*} M\right) \rightarrow p^{*} \mathcal{H}^{l}\left(f_{*} K\right)[d]$ is iso for $l \geqslant 2$ and epi for $l=1$.

Proof. - Since $p^{\prime *}[d]$ is $t$-exact, (i) follows from Lemma 4.7.6(i) applied to $K^{\prime}$ in the set-up of 4.7, keeping in mind that $f_{*}^{\prime} p^{\prime *} \simeq p^{*} f_{*}$ and $p^{*}[d]\left({ }^{p} \mathcal{H}^{l}\left(f_{*} K\right)\right) \simeq{ }^{p} \mathcal{H}^{l}\left(p^{*}[d]\left(f_{*} K\right)\right)$.

By transversality, 4.7.1 and Lemma 3.5.4(b), $i^{!} K^{\prime} \simeq i^{*} K^{\prime}[2]$ and (ii) follows from Lemma 4.7.6(ii).

The following complements Proposition 4.7.8; see [1, 5.4.11].

PROPOSITION 4.7.9. $-p^{*}[d]^{p} \mathcal{H}^{-1}\left(f_{*} K\right)$ can be identified with the biggest perverse subsheaf of ${ }^{p} \mathcal{H}^{0}\left(g_{*} M\right)$ coming from $Y$, and $p^{*}[d]^{p} \mathcal{H}^{1}\left(f_{*} K\right)$ with the biggest quotient perverse sheaf of ${ }^{p} \mathcal{H}^{0}\left(g_{*} M\right)$ coming from $Y$.

\section{Proof of the main theorems in 2.1}

In this section, we prove Theorem 2.1.1, i.e. the Relative Hard Lefschetz Theorem, the Decomposition Theorem and the Semisimplicity Theorem (except for ${ }^{p} \mathcal{H}^{0}\left(f_{*} \mathbb{Q}_{X}[n]\right)$ ), the Hard Lefschetz Theorem for Perverse Cohomology 2.1.4, the Hodge Structure Theorem 2.1.5, the $(\eta, L)$ Decomposition Theorem 2.1.7 and the Generalized Hodge-Riemann Bilinear Relations 2.1.8.

The set-up is the one of 2.1. We assume 2.6.2 and remind the reader of Remark 2.6.3.

\subsection{Relative Hard Lefschetz, Decomposition and Semisimplicity $(i \neq 0)$}

We use the notation and results in 4.7. Let $\eta^{\prime}:=i^{*} p^{\prime *} \eta$; it is $g$-ample.

LEMmA 5.1.1. - Suppose that $\eta^{\prime r}:{ }^{p} \mathcal{H}^{-r}\left(g_{*} M\right) \stackrel{\simeq}{\longrightarrow} \mathcal{H}^{r}\left(g_{*} M\right)$ for all $r \geqslant 0$ and that ${ }^{p} \mathcal{H}^{0}\left(g_{*} M\right)$ is semisimple. Then $\eta^{r}:{ }^{p} \mathcal{H}^{-r}\left(f_{*} K\right) \stackrel{\simeq}{\longrightarrow} \mathcal{H}^{r}\left(f_{*} K\right)$ for $r \geqslant 0$.

Proof. - We have $\eta^{r}=i_{*} \circ \eta^{\prime r-1} \circ i^{*}$. If $r \neq 1$, then we conclude by the Weak Lefschetz Theorem 4.7.8.

Let $r=1$. Since $p^{*}[d]$ is fully faithful, $\eta$ is an isomorphism if and only if $p^{\prime *} \eta[d]: p^{* p} \mathcal{H}^{-1}\left(f_{*} K\right)[d] \rightarrow p^{*} \mathcal{H}^{1}\left(f_{*} K\right)[d]$ is an isomorphism. This map is the composition of the monomorphism $i^{*}$ with the epimorphism $i_{*}$. By the semisimplicity of ${ }^{p} \mathcal{H}^{0}\left(g_{*} M\right)$, the sequence of perverse subsheaves $i^{*} \operatorname{Ker} p^{*} \eta[d] \subseteq \operatorname{Ker} i_{*} \subseteq{ }^{\mathcal{}} \mathcal{H}^{0}\left(g_{*} M\right)$ splits and we get a direct 
sum decomposition

$$
{ }^{p} \mathcal{H}^{0}\left(g_{*} M\right)=i^{*} \operatorname{Ker} p^{\prime *} \eta[d] \oplus R \oplus S,
$$

where the restriction of $i_{*}$ to $S$ is an isomorphism with $p^{* p} \mathcal{H}^{1}\left(f_{*} K\right)[d]$.

The projection ${ }^{P} \mathcal{H}^{0}\left(g_{*} M\right) \rightarrow i^{*} \operatorname{Ker} p^{\prime *} \eta[d] \oplus S \simeq i^{*} \operatorname{Ker} p^{\prime *} \eta[d] \oplus p^{*} \mathcal{H}^{1}\left(f_{*} K\right)[d]$ is an epimorphism and both summands come from $Y$. By the maximality statement of Proposition 4.7.9, $i^{*} \operatorname{Ker} p^{*} \eta[d]=0$, i.e. $\eta:^{p} \mathcal{H}^{-1}\left(f_{*} K\right) \rightarrow^{p} \mathcal{H}^{1}\left(f_{*} K\right)$ is a monomorphism.

Since the complex $K$ and the map $\eta$ are self-dual, $\eta$ is also an epimorphism, hence an isomorphism.

Remark 5.1.2. - The relative Hard Lefschetz Theorem 2.1.1 holds when $\eta$ is $f$-ample, i.e. ample when restricted to the fibers of $f$. In fact, if $\eta$ is $f$-ample, then $\tilde{\eta}:=\eta+m L$ is ample for every $m \gg 0$ and, by Remark 4.4.3, ${ }^{p} \mathcal{H}^{j}(\eta)={ }^{p} \mathcal{H}^{j}(\tilde{\eta})$, for every $j \in \mathbb{Z}$. Note that ample implies $f$-ample. We need Theorem 2.1.1 for $f$-ample line bundles in the next proposition.

We can now prove Theorem 2.1.1 parts (a)-(c), except for $i=0$.

Proposition 5.1.3. - Let $f: X \rightarrow Y$ and $\eta$ be as in 2.1 and assume that 2.6.2 holds.

Then the Relative Hard Lefschetz Theorem 2.1.1(a) and the Decomposition Theorem 2.1.1(b) hold for $f$. The Semisimplicity Theorem 2.1.1(c) holds for ${ }^{P} \mathcal{H}^{i}\left(f_{*} \mathbb{Q}_{X}[n]\right)$ with $i \neq 0$.

Proof. - We apply the inductive hypothesis 2.6.2 to $g: \mathcal{X} \rightarrow \mathcal{Y}$, which satisfies $r(g)<r(f)$ (cf. 4.7.4). Setting $K=\mathbb{Q}_{X}[n]$, we have $M=\mathbb{Q}_{\mathcal{X}}[n+d-1]$. By the inductive hypothesis and Remark 5.1.2, we have: (1) $\eta^{\prime r}$ is an isomorphism for every $r$ and (2) ${ }^{p} \mathcal{H}^{0}\left(g_{*} M\right)$ is semisimple. By Lemma 5.1.1, $\eta^{r}$ is an isomorphism for $r \geqslant 0$. This proves that Theorem 2.1.1(a) holds for $f$. As it has already been observed in 4.4.4, the well-known Deligne-Lefschetz Criterion for $E_{2}$-degeneration [9] yields Theorem 2.1.1(b) for $f$. The semisimplicity statement (b) for $i \neq 0$ follows from the Weak Lefschetz Proposition 4.7.8 and the semisimplicity of ${ }^{p} \mathcal{H}^{0}\left(g_{*} M\right)$.

\subsection{Hard Lefschetz for perverse cohomology groups}

Note that $r(f) \leqslant \operatorname{dim} X$. If $r(f)>0$, consider $1 \leqslant k \leqslant r(f)$, let $X^{k}$, be the transversal intersection of $k$ general hyperplane sections of the linear system $\eta$ and $f_{k}: X^{k} \rightarrow Y$ be the resulting map. By Proposition 4.7.5, we may assume that $\eta$ is such that if $r(f)>0$, then $r\left(f_{k}\right) \leqslant r(f)-k$, for every $1 \leqslant k \leqslant r(f)$.

The following contains weak-Lefschetz-type results for the hyperplane sections of $X$.

Proposition 5.2.1. - Assumptions as in 5.2. Let $1 \leqslant k \leqslant r$.

(i) The natural restriction map ${ }^{p} \mathcal{H}^{l-k}\left(f_{*} \mathbb{Q}_{X}[n]\right) \rightarrow{ }^{p} \mathcal{H}^{l}\left(f_{k_{*}} \mathbb{Q}_{X^{k}}[n-k]\right)$ is iso for $l<0$ and a splitting mono for $l=0$.

(ii) The natural Gysin map ${ }^{p} \mathcal{H}^{l}\left(f_{k_{*}} \mathbb{Q}_{X^{k}}[n-k]\right) \rightarrow{ }^{p} \mathcal{H}^{k+l}\left(f_{*} \mathbb{Q}_{X}[n]\right)$ is an iso for $l>0$ and a splitting epi for $l=0$.

(iii) The maps induced by $L$ in hypercohomology are compatible with the splittings (i) and (ii). In particular, if for given $h$ and $j$ the map

$$
L^{j}: H_{0}^{h}\left(X^{k}\right) \rightarrow H_{0}^{h+2 j}\left(X^{k}\right)
$$

is injective (respectively surjective, respectively bijective), then the maps

$$
L^{j}: H_{-k}^{h}(X) \rightarrow H_{-k}^{h+2 j}(X), \quad L^{j}: H_{k}^{h+2 k}(X) \rightarrow H_{k}^{h+2 k+2 j}(X),
$$

are injective (respectively surjective, respectively bijective).

$4^{\mathrm{e}}$ SÉRIE - TOME $38-2005-\mathrm{N}^{\circ} 5$ 
Proof. - Let $k=1$. Lemma 4.7.6 applied to $X^{1} \subseteq X$ and $\mathbb{Q}_{X}[n]$ implies (i) and (ii). The case $k>1$ follows by induction.

Since cupping with $L=f^{*} A$ commutes with any direct sum decomposition on $Y$, (iii) follows.

We need the following easy consequence of Proposition 5.2.1 to prove Theorem 2.1.4. The more precise statement $\operatorname{Ker} L_{n-1} \subseteq H_{\leqslant-1}^{n-1}(X)$ is true, but its proof would require that we prove Theorem 2.1.4 first.

LEMMA 5.2.2. - Assumptions as in 5.2.

$$
\operatorname{Ker} L_{n-1}=\operatorname{Ker} L_{n-1, \leqslant 0} \subseteq H_{\leqslant 0}^{n-1}(X) .
$$

Proof. - Since $L$ acts compatibly with the $p$-splitting, it suffices to prove that $\operatorname{Ker} L_{n-1} \subseteq$ $H_{k}^{n-1}(X)$ is trivial, for every $k>0$. By Proposition 5.2.1, $H_{k}^{n-1}(X)$ is isomorphic to a direct summand of $H_{0}^{(n-k)-(k+1)}\left(X^{k}\right)$ with $L$ acting as the restriction of $L_{\mid X^{k}}$ to the direct summand. Inductively, this map is injective. We conclude by Proposition 5.2.1(iii).

PROPOSITION 5.2.3. - Under the assumption 2.6.2 the Hard Lefschetz theorem for perverse cohomology groups 2.1.4 holds for $f$, i.e.

$$
\eta^{k}: H_{-k}^{j}(X) \simeq H_{k}^{j+2 k}(X), \quad L^{k}: H_{b}^{n+b-k}(X) \simeq H_{b}^{n+b+k}(X), \quad k \geqslant 0, \quad b, j \in \mathbb{Z} .
$$

Proof. - Since $f_{*} \mathbb{Q}_{X}[n] p$-splits by 5.1.3, there is a decomposition

$$
H_{\leqslant b}^{n+j}(X) \stackrel{\varphi}{\simeq} \bigoplus_{i \leqslant b} \mathbb{H}^{j-i}\left(Y,{ }^{p} \mathcal{H}^{i}\left(f_{*} \mathbb{Q}_{X}[n]\right)\right) .
$$

The statement for $\eta^{k}$ follows from the previously established Relative Hard Lefschetz Theorem for $f$ (cf. 5.1.3) and from the compatibility 4.4.2.

The rest of the proof is concerned with $L^{k}$. The cases $b \neq 0$ follow from the inductive hypotheses: apply Theorem 2.1.4 and Proposition 5.2.1(iii) to $f_{\mid X^{k}}: X^{k} \rightarrow Y$.

Let $b=0$. Note that the statement is trivial for $k=0$. Choose a sufficiently general hyperplane section $Y_{1}$ of $Y$ (cf. 3.2.2) and let $X_{1}=f^{-1}\left(Y_{1}\right)$, which, by Bertini Theorem we can assume nonsingular.

Recalling the canonical identification $H_{0}^{n+k}(X)=\mathbb{H}^{-k}\left(Y,{ }^{p} \mathcal{H}^{0}\left(f_{*} \mathbb{Q}_{X}[n]\right)\right)$ and the compatibility 4.4.3, we have a commutative diagram

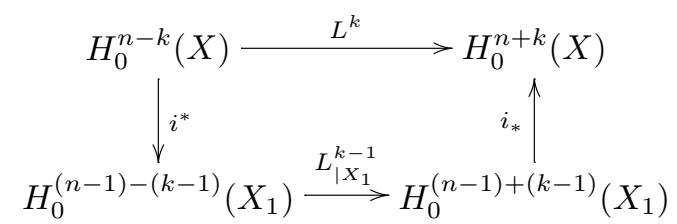

where $i^{*}$ is restriction and $i_{*}$ is the (dual) Gysin map.

Let $k \geqslant 2$. By Proposition 4.7.7, $i^{*}$ and $i_{*}$ are isomorphisms. By Theorem 2.1.4 applied to $f_{1}: X_{1} \rightarrow Y_{1}$, the map $L_{\mid X_{1}}^{k-1}$ is an isomorphism and so is the map $L^{k}$.

Let $k=1$. We must check that $L: H_{0}^{n-1}(X) \rightarrow H_{0}^{n+1}(X)$ is an isomorphism.

By the self-duality of $p \mathcal{H}^{0}\left(f_{*} \mathbb{Q}_{X}[n]\right)$, the two spaces have the same dimension so that it enough to check that $L$ is injective. 
Let $\alpha \in \operatorname{Ker} L \subseteq H_{0}^{n-1}(X)$. According to 4.4.4, $\alpha=\sum_{j \geqslant 0} \eta^{j} \alpha_{j}$. By Remark 4.4.3, $L$ commutes with any direct sum decomposition so that $L \eta^{j} \alpha_{j}=0$, for every $j \geqslant 0$. Since $L$ commutes with $\eta$ and, by what we have already proved for $\eta^{k}$, the map $\eta^{j}$ is injective on $H_{-2 j}^{n-2 j-1}(X) \ni \alpha_{j}$ for every $j \geqslant 0$, we have that $L \alpha_{j}=0$ for every $j \geqslant 0$. The case $b \neq 0$, which was dealt-with above, implies that if $j>0$, then $L$ is an isomorphism from $H_{-2 j}^{n-2 j-1}(X)$. This implies that $\alpha_{j}=0$, for every $j>0$, i.e. $\alpha=\alpha_{0}$.

Since $L$ acting on $H^{*}(X)$ is strict and $\operatorname{Ker} L \subseteq H_{\leqslant 0}^{n-1}(X)$ by Lemma 5.2.2, we have $\operatorname{Ker} L /\left(\operatorname{Ker} L \cap H_{\leqslant-1}^{n-1}\right)=\operatorname{Ker}\left\{H_{0}^{n-1}(X) \stackrel{L}{\rightarrow} H_{0}^{n+1}(X)\right\}$. It follows that there exists $a \in$ Ker $L \subseteq H_{\leqslant 0}^{n-1}(X)$ such that its class in $H_{0}^{n-1}(X)$ satisfies $[a]=\alpha$.

Let $a=\sum a^{p q}$ be the $(p, q)$-decomposition for the natural Hodge structure on $H^{n-1}(X)$. Clearly $a^{p q} \in \operatorname{Ker} L$. It follows that we may assume that $\alpha=[a]$, with $a \in \operatorname{Ker} L$ of some pure type $(p, q)$.

By way of contradiction, assume that $\alpha \neq 0$. By Lemma 4.3.8, we have $i^{*} \alpha=\left[a_{\mid X_{1}}\right]$. Since $i^{*}$ is injective by Proposition 4.7.7, $0 \neq i^{*} \alpha \in H_{0}^{n-1}\left(X_{1}\right)$. This restricted class is of pure type $(p, q)$, for the pure Hodge structure coming inductively from Theorem 2.1.5 applied to $f_{\mid X_{1}}: X_{1} \rightarrow Y_{1}$. By Lemma 4.3.8: (i) $L_{\mid X_{1}} i^{*} \alpha=i^{*}(L \alpha)$ and (ii) since $\left(\mathcal{P}_{\eta}\right)_{\mid X_{1}} \simeq \mathcal{P}_{\eta_{\mid X_{1}}}$ [1], we have $\eta_{\mid X_{1}} i^{*} \alpha=0$. Since $\alpha=\alpha_{0}, i^{*} \alpha \in P_{0}^{0}\left(X_{1}\right)$.

By the inductive Generalized Hodge-Riemann Relations 2.1.8 for $f_{\mid X_{1}}: X_{1} \rightarrow Y_{1}$

$$
0 \neq \pm S_{00}^{\eta_{\mid} L_{\mid}}\left(i^{*} \alpha, \overline{i^{*} \alpha}\right)=\int_{X_{1}} a_{\mid X_{1}} \wedge \bar{a}_{\mid X_{1}}=\int_{X} L \wedge a \wedge \bar{a}=0
$$

a contradiction.

Proposition 5.2.3 allows to complete the identification of the filtrations defined in 4.5:

Proposition 5.2.4. - For every $i, j \in \mathbb{Z}$ :

$$
\begin{aligned}
& W^{\eta}=W^{\mathrm{deg}}, \quad W^{L}=W^{\mathrm{tot}}, \\
& W_{i}^{L}=\bigoplus_{l \in \mathbb{Z}} H_{\leqslant l+i}^{n+l}(X), \quad G r_{i}^{L}=\bigoplus_{l \in \mathbb{Z}} H_{l+i}^{n+l}(X), \quad W_{i}^{L} \cap H^{n+j}(X)=H_{\leqslant i+j}^{n+j}(X), \\
& G r_{j+i}^{\eta} G r_{j}^{L} H^{*}(X)=H_{-i}^{n-i-j}(X)
\end{aligned}
$$

and the forms $S_{i j}^{\eta L}$ of (30) are therefore defined on $H_{-i}^{n-i-j}(X)$.

The filtration $W^{\eta}[j]$ is the weight-j filtration of $\eta$ relative to $W^{L}(c f .(28))$.

Proof. - Since $L$ is compatible with the splitting $H^{*}(X)=\bigoplus_{l} H^{l}(X)$, the convolution formula (23) implies that

$$
W_{i}^{L}=\bigoplus_{l}\left(W_{i}^{L} \cap H^{l}(X)\right) .
$$

By the characterization of weight filtrations, in order to prove that $W^{\text {deg }}=W^{\eta}$ and $W^{\text {tot }}=W^{L}$, it is enough to show that

(i) $\eta W_{k}^{\text {deg }} \subseteq W_{k-2}^{\text {deg }}$,

(ii) $\eta^{k}: H^{n-k}(X) \simeq H^{n+k}(X)$,

(iii) $L W_{k}^{\text {tot }} \subseteq W_{k-2}^{\text {tot }}$, and

(iv) $L^{k}: G r_{k}^{\text {tot }} \simeq G r_{-k}^{\text {tot }}$, for all $k \geqslant 0$.

$4^{\mathrm{e}}$ SÉRIE - TOME $38-2005-\mathrm{N}^{\circ} 5$ 
While (i) is obvious and (iii) follows from 4.4.3, (ii) and (iv) are mere re-formulations of the Hard Lefschetz Theorems 3.1.2 for $\eta^{k}$ acting on ordinary cohomology and of the just-established Hard Lefschetz for Perverse Cohomology Theorem 2.1.4 for the action of $L^{k}$, respectively. This proves (38), (39) and (40). The assertion on $S^{\eta L}$ follows from (40) and the definition (30).

The second assertion follows from $W^{L}=W^{\text {deg }}$, (i) above and the Hard Lefschetz Theorem 2.1.4 for $\eta$.

Proof of the Hodge Structure Theorem 2.1.5. - Since $L$ is of pure type $(1,1)$ acting on $H^{*}(X)$, the convolution formula (23) for $W^{L}$ implies that $W_{i}^{L} \cap H^{n+j}(X)$ is a Hodge sub-structure of $H^{n+j}(X)$ for every $i, j \in \mathbb{Z}$. We conclude by (39).

Proof of the ( $\eta, L)$-Decomposition Corollary 2.1.7. - By Proposition 5.2.4, $W^{\eta}[j]$ is the weight $-j$ filtration of $\eta$ relative to $W^{L}$ and 4.5 applies.

\subsection{The Generalized Hodge-Riemann Bilinear Relations $\left(P_{j}^{i} \neq P_{0}^{0}\right)$}

In this section we are going to prove the Generalized Hodge-Riemann Bilinear Relations 2.1.8 for $f$, except for $P_{0}^{0}$. As in 5.2, we may assume that the $\eta$-sections are general enough.

LEMMA 5.3.1.-

(a) Let $r>0$ and $X^{r}$ be the complete transversal intersection of $r$ general sections of $\eta$. Then the natural restriction map $i^{*}: H_{-r}^{n-r-j}(X) \rightarrow H_{0}^{n-r-j}\left(X^{r}\right)$, is an injective map of pure Hodge structures for every $j \in \mathbb{Z}$, and $i^{*}\left(P_{-r}^{-j}(X)\right) \subseteq P_{0}^{-j}\left(X^{r}\right)$ for every $j \geqslant 0$.

(b) Let $X_{1}=f^{-1}\left(Y_{1}\right)$, where $Y_{1}$ is a general section of $A$, transversal to the strata of $Y$. Then, for every $j>0$, the natural restriction map $i^{*}: H_{0}^{n-j}(X) \rightarrow H_{0}^{n-j}\left(X_{1}\right)$ is an injection of pure Hodge structures and $i^{*}\left(P_{0}^{-j}(X)\right) \subseteq P_{0}^{-j+1}\left(X_{1}\right)$.

Proof. - The inductive hypotheses apply to $X^{r} \rightarrow Y$ and to $X_{1} \rightarrow Y_{1}$ so that all perverse cohomology groups have natural Hodge structures.

(a) We have proved Theorem 2.1.5 for $f$. The map $H^{*}(X) \rightarrow H^{*}\left(X^{r}\right)$ is a map of Hodge structures and Remark 4.2.3 implies that so is the map in question. Lemma 5.2.1(i) implies the injectivity statement. The fact that $P_{-r}^{-j}(X)$ maps to $P_{0}^{-j}\left(X^{r}\right)$ can be shown as follows. Let $l>0$ and $l^{\prime} \geqslant 0$ and let $X^{l}$ be the transversal complete intersection of $l$ general sections of $\eta$. The map $f_{*}\left(\eta^{l+l^{\prime}}\right): f_{*} \mathbb{Q}_{X}[n] \rightarrow f_{*} \mathbb{Q}_{X}\left[n+2 l+2 l^{\prime}\right]$ factorizes as

$$
f_{*} \mathbb{Q}_{X}[n] \rightarrow g_{*} \mathbb{Q}_{X^{l}}[n-l][l] \stackrel{g_{*}\left(\eta_{\mid X}^{l^{\prime}}\right)}{\longrightarrow} g_{*} \mathbb{Q}_{X^{l}}[n-l][l]\left[2 l^{\prime}\right] \rightarrow f_{*} \mathbb{Q}_{X}\left[n+2 l+2 l^{\prime}\right] .
$$

The statement follows from applying the functors $\mathbb{H}^{*}\left(\mathcal{H}^{-r}(-)\right)$ to the factorization above when $l=r$ and $l^{\prime}=1$.

(b) The compatibility with the perverse decomposition of Lemma 4.3.8 implies that the map in question has the indicated range. The map $H^{*}(X) \rightarrow H^{*}\left(X_{1}\right)$ is a map of Hodge structures and so is the map in question. The injectivity statement follows from Proposition 4.7.7. The fact that $P_{0}^{-j}(X)$ maps to $P_{0}^{-j+1}\left(X_{1}\right)$ follows from the fact that $L^{j}=i_{*} \circ L_{\mid X_{1}}^{j-1} \circ i^{*}$, where $i_{*}$ is an isomorphism in the range we are using it.

Proposition 5.3.2. - The Generalized Hodge-Riemann Bilinear Relations 2.1.8 hold for the direct summands $\eta^{-i+l} L^{-j+m} P_{i-2 l}^{j-2 m} \neq P_{0}^{0}$.

Proof. - By Remark 4.5.2, it is enough to consider the case $i, j \geqslant 0,(i, j) \neq(0,0)$. Also, we assume that $l=m=0$, and leave the easy necessary modifications to deal with the other cases to the reader. 
Let $X_{s}^{r}$ be the complete intersection of $r$ general sections of $\eta$ and $s$ general sections of $L$. If $r=0$, then we consider only the sections of $L$. Similarly, if $s=0$.

Since $\int_{X} \eta^{r} \wedge L^{s} \wedge a \wedge b=\int_{X_{s}^{r}} a_{\mid X_{s}^{r}} \wedge b_{\mid X_{s}^{r}}$, we have that

$$
S_{r s}^{\eta L}(X)(a, b)=S_{00}^{\eta_{\mid} L_{\mid}}\left(X_{s}^{r}\right)\left(a_{\mid X_{s}^{r}}, b_{\mid X_{s}^{r}}\right) .
$$

The statement follows from a repeated application of Lemma 5.3.1, of the inductive hypothesis Theorem 2.1.8 applied to $X_{s}^{r} \rightarrow Y_{s}$ and from Remark 3.1.1.

\subsection{The space $\Lambda \subseteq H^{n}(X)$, its approximability and the polarization of $P_{0}^{0}$}

In this section we are going to complete the proof of the Generalized Hodge-Riemann Bilinear Relations 2.1 .8 for $f$, by polarizing $P_{0}^{0}$. For convenience, we consider cohomology with real coefficients.

Let $\varepsilon>0$ be a real number. Define

$$
\Lambda_{\varepsilon}:=\operatorname{Ker}(\varepsilon \eta+L) \subseteq H^{n}(X) .
$$

The spaces $\Lambda_{\varepsilon}$ are Hodge sub-structures. By the classical Hard Lefschetz Theorem, $\operatorname{dim} \Lambda_{\varepsilon}=$ $b_{n}-b_{n-2}$, where $b_{i}$ are the Betti numbers of $X$. Define

$$
\Lambda:=\lim _{\varepsilon \rightarrow 0} \Lambda_{\varepsilon}
$$

where the limit is taken in the Grassmannian $G\left(b_{n}-b_{n-2}, H^{n}(X)\right)$. The space $\Lambda \subseteq H^{n}(X)$ is a real Hodge sub-structure for that is a closed condition. Clearly, $\Lambda \subseteq \operatorname{Ker}\left\{H^{n}(X) \stackrel{L}{\longrightarrow}\right.$ $\left.H^{n+2}(X)\right\}$, but in general there is no equality, since, by counting dimensions,

$$
\operatorname{dim} \operatorname{Ker}\left\{H^{n}(X) \stackrel{L}{\longrightarrow} H^{n+2}(X)\right\}=b_{n}-b_{n-2}+\operatorname{dim} \operatorname{Ker}\left\{H^{n-2}(X) \stackrel{L}{\longrightarrow} H^{n}(X)\right\} .
$$

The main goal of this section is to characterize the subspace $\Lambda$ in terms of $\eta$ and $L$.

Since $L^{k}: G r_{k}^{L} \simeq G r_{-k}^{L}$, we have

$$
\operatorname{Ker} L^{k} \subseteq W_{k-1}^{L}
$$

In order to keep track of cohomological degrees, we set

$$
L_{r}^{k}: G r_{r}^{\eta}=H^{n-r}(X) \rightarrow H^{n-r+2 k}(X)=G r_{r-2 k}^{\eta} .
$$

The following two lemmata will allow to identify $\Lambda$.

LEMmA 5.4.1. - $\eta \operatorname{Ker} L_{2} \cap\left(\eta \operatorname{Ker} L_{2}\right)^{\perp} \cap \cdots \cap\left(\eta^{i} \operatorname{Ker} L_{2 i}^{i}\right)^{\perp}=\{0\} \in H^{n}(X), i \gg 0$.

Proof. - It is enough to show that

$$
\eta \operatorname{Ker} L_{2} \cap\left(\eta \operatorname{Ker} L_{2}\right)^{\perp} \cap \cdots \cap\left(\eta^{i} \operatorname{Ker} L_{2 i}^{i}\right)^{\perp}=\eta \operatorname{Ker} L_{2} \cap W_{-i}^{L}, \quad \forall i \geqslant 0,
$$

for then the lemma follows by taking $i=n+1$, for example.

The claim above can be proved by induction as follows. The starting step $i=0$ of the induction follows from (41):

$$
\operatorname{Ker} L_{2} \subseteq W_{0}^{L}
$$

$4^{\text {e }}$ SÉRIE - TOME $38-2005-\mathrm{N}^{\circ} 5$ 
Suppose the claim proved for $i$. We are left with showing that

$$
\eta \operatorname{Ker} L_{2} \cap W_{-i-1}^{L}=\eta \operatorname{Ker} L_{2} \cap W_{-i}^{L} \cap\left(\eta^{i+1} \operatorname{Ker} L_{2 i+2}^{i+1}\right)^{\perp} .
$$

The inclusion "ᄃ" follows at once from (41) and self-duality: $\eta^{i+1} \operatorname{Ker} L_{2 i+2}^{i+1} \subseteq W_{i}^{L}=$ $\left(W_{-i-1}^{L}\right)^{\perp}$. The other inclusion follows from the nondegeneracy of the forms $S_{i+2, i}^{\eta L}$, as we now show.

Let $\alpha=\eta \lambda \in \eta \operatorname{Ker} L_{2} \cap W_{-i}^{L}$.

Claim 1. - $\lambda \in W_{-i}^{L}$.

We have $\lambda \in W_{0}^{L}$. By way of contradiction, assume that $\lambda \in W_{-i^{\prime}}^{L}$, for some $-i<-i^{\prime} \leqslant 0$. Since $\eta \lambda \in W_{-i}^{L}$, we have $\eta \lambda \in H_{\leqslant-i}^{n}(X)$ and we would have that the map

$$
\eta: G r_{2}^{\eta} G r_{-i^{\prime}}^{L}=H_{-i^{\prime}-2}^{n-2}(X) \rightarrow G r_{0}^{\eta} G r_{-i^{\prime}}^{L}=H_{-i^{\prime}}^{n}
$$

is not injective, contradicting Proposition 5.2.4, i.e. the injectivity of $\eta$ for $i \geqslant-1$.

CLAIM 2. - There exists $\lambda^{\prime} \in W_{2 i+2}^{\eta} \cap W_{i}^{L}=H_{\leqslant-2-i}^{n-2-2 i}(X)$ such that $\lambda=L^{i} \lambda^{\prime}$.

We have that $L^{k}: G r_{k}^{L} \simeq G r_{-k}^{L}$ for every $k \geqslant 0$. Using the case $k=i$ we may write

$$
\lambda=L^{i} \lambda_{1}+\tau_{1}, \quad \lambda_{1} \in H_{\leqslant-2-i}^{n-2-2 i}(X), \tau_{1} \in H_{\leqslant-2-i-1}^{n-2}(X) .
$$

Replacing $\lambda$ with $\tau_{1}, k=i$ with $k=i+1$ and iterating we get

$$
\lambda=L^{i} \sum_{t=1}^{j} L^{t-1} \lambda_{t}+\tau_{j}, \quad L^{t-1} \lambda_{t} \in H_{\leqslant-2-i}^{n-2-2 i}(X), \tau_{j} \in H_{\leqslant-2-i-j}^{n-2}(X) .
$$

Claim 2 follows by taking $j \gg 0$.

Since $L_{2} \lambda=0$, we have $L_{2 i+2}^{i+1} \lambda^{\prime}=0$. So far, we have proved that

$$
\text { if } \alpha \in \eta \operatorname{Ker} L \cap W_{-i}^{L}, \quad \text { then } \alpha=\eta L_{2 i+2}^{i} \lambda^{\prime} \text { with } L_{2 i+2}^{i+1} \lambda^{\prime}=0 .
$$

Let $\beta \in \eta^{i+1} \operatorname{Ker} L_{2 i+2}^{i+1}$. By (41), $\beta=\eta^{i+1} \beta^{\prime}$, for some $\beta^{\prime} \in \operatorname{Ker} L_{2 i+2}^{i+1} \subseteq W_{i}^{L}$. By the very definition (30) of the forms $S^{\eta L}$ it follows that, denoting by the same symbol an element in some $H_{\leqslant *}^{*}$ and the corresponding class in $H_{*}^{*}(X)$,

$$
S_{i j}^{\eta L}\left(\lambda^{\prime}, \beta^{\prime}\right)=S\left(\eta L^{i} \lambda^{\prime}, \eta^{i+1} \beta^{\prime}\right)=S(\alpha, \beta) .
$$

Finally, let us assume that $\alpha \in \eta \operatorname{Ker} L_{2} \cap W_{-i}^{L} \cap\left(\eta^{i+1} \operatorname{Ker} L_{2 i+2}^{i+1}\right)^{\perp}$. This implies $S(\alpha, \beta)=0$.

By the Generalized Hodge-Riemann Bilinear Relations 2.1.8, the restriction of the form $S_{i+2, i}^{\eta L}$ to $\operatorname{Ker} L^{i+1} \subseteq H_{-i-2}^{n-2 i-2}(X)$ is nondegenerate. It follows that the class of $\lambda^{\prime}$ in $H_{-i-2}^{n-2 i-2}(X)$ is zero, i.e. $\lambda^{\prime} \in W_{i-1}^{L}$. Since $\eta$ respects the filtration $W^{L}$ while $L$ shifts it by -2 , we conclude that $\alpha=\eta L^{i} \lambda^{\prime} \in W_{-i-1}^{L}$. We have proved the remaining inclusion.

LEMMA 5.4.2. $-\Lambda=\operatorname{Ker} L_{0} \cap\left(\bigcap_{i \geqslant 1}\left(\eta^{i} \operatorname{Ker} L_{2 i}^{i}\right)^{\perp}\right) \subseteq H_{\leqslant 0}^{n}(X)$ and there is the orthogonal direct sum decomposition:

$$
\operatorname{Ker} L_{0}=\Lambda \oplus \eta \operatorname{Ker} L_{2} \text {. }
$$


Proof. - We show that

$$
\Lambda_{\varepsilon} \subseteq \bigcap_{i \geqslant 1}\left(\eta^{i} \operatorname{Ker} L_{2 i}^{i}\right)^{\perp}
$$

It is enough to show that $\Lambda_{\varepsilon} \subseteq\left(\eta^{i} \operatorname{Ker} L_{2 i}^{i}\right)^{\perp}$, for every $i \geqslant 1$. Let $u_{\varepsilon} \in \Lambda_{\varepsilon}$, i.e. $\eta u_{\varepsilon}=-\varepsilon^{-1} L u_{\varepsilon}$. We have $\eta^{i} u_{\varepsilon}=\left(-\varepsilon^{-1}\right)^{i} L^{i} u_{\varepsilon}$. Let $\lambda \in H^{n-2 i}(X)$ be such that $L^{i} \lambda=0$. We have $\int_{X} u_{\varepsilon} \wedge \eta^{i} \lambda=$ $\left(-\frac{1}{\varepsilon}\right)^{i} \int_{X} u_{\varepsilon} \wedge L^{i} \lambda=0$. The wanted inclusion follows and $\Lambda \subseteq \bigcap_{i \geqslant 1}\left(\eta^{i} \operatorname{Ker} L_{2 i}^{i}\right)^{\perp}$.

We show that $\Lambda \subseteq \operatorname{Ker} L_{0}$ : if $\Lambda \ni u=\lim _{\varepsilon \rightarrow 0} u_{\varepsilon}$, with $u_{\varepsilon} \in \Lambda_{\varepsilon}$, then $L u=\lim _{\varepsilon \rightarrow 0} L u_{\varepsilon}=$ $\lim _{\varepsilon \rightarrow 0}\left(-\varepsilon \eta u_{\varepsilon}\right)=0$.

It follows that $\Lambda \subseteq \operatorname{Ker} L_{0} \cap\left(\cap_{i \geqslant 1}\left(\eta^{i} \operatorname{Ker} L_{2 i}^{i}\right)^{\perp}\right)$.

By Lemma 5.4.1, $\bigcap_{i \geqslant 1}\left(\eta^{i} \operatorname{Ker} L_{2 i}^{i}\right)^{\perp} \cap \eta \operatorname{Ker} L_{2}=\{0\}$ and therefore $\Lambda \cap \eta \operatorname{Ker} L_{2}=\{0\}$. By counting dimensions, the internal direct sum $\Lambda \oplus \eta \operatorname{Ker} L_{2}=\operatorname{Ker} L_{0}$. On the other hand, we also have an internal direct sum $\left(\operatorname{Ker} L_{0} \cap\left(\bigcap_{i \geqslant 1}\left(\eta^{i} \operatorname{Ker} L_{2 i}^{i}\right)^{\perp}\right)\right) \oplus \eta \operatorname{Ker} L_{2} \subseteq \operatorname{Ker} L_{0}$ and this implies that the inclusion $\Lambda \subseteq \operatorname{Ker} L_{0} \cap\left(\bigcap_{i \geqslant 1}\left(\eta^{i} \operatorname{Ker} L_{2 i}^{i}\right)^{\perp}\right)$ is in fact an equality. The orthogonality of the decomposition is immediate.

The form $S_{00}^{\eta L}$ is nondegenerate on each direct summand of the $(\eta, L)$-decomposition for $H_{0}^{n}(X)$. In particular, it is so on $P_{0}^{0}$.

LEMmA 5.4.3. - The form $(-1)^{n} S_{00}^{\eta L}$ defines a polarization of $\Lambda_{0}:=\Lambda /\left(\Lambda \cap H_{\leqslant-1}^{n}(X)\right)$.

Proof. - By the classical Hard Lefschetz Theorem, the Poincaré pairing multiplied by $(-1)^{\frac{n(n+1)}{2}}$ is a polarization of $\Lambda_{\varepsilon}$ for every $\varepsilon>0$. In particular, the form $(-1)^{n} S(-, C(-))$ is semipositive definite when restricted to $\Lambda$.

It follows that $(-1)^{n} S_{00}^{\eta L}(-, C(-))$, being semipositive definite and nondegenerate on $P_{0}^{0}$, is in fact positive definite, i.e. $(-1)^{n} S_{00}^{\eta L}$ is a polarization of $P_{0}^{0}$.

LEMMA 5.4.4. - We have an orthogonal direct sum decomposition:

$$
\operatorname{Ker} L_{0} /\left(\operatorname{Ker} L_{0} \cap H_{\leqslant-1}^{n}(X)\right)=\Lambda_{0} \oplus\left(\eta \operatorname{Ker} L_{2} / \eta \operatorname{Ker} L_{2} \cap H_{\leqslant-1}^{n}(X)\right) .
$$

Proof. - The statement follows from the following elementary fact: if $V$ is a vector space with a bilinear form and $V_{1} \subseteq V$ is its radical, an orthogonal direct sum decomposition

$$
V=U_{1} \oplus U_{2}
$$

induces an orthogonal direct sum decomposition

$$
V / V_{1}=U_{1} /\left(V_{1} \cap U_{1}\right) \oplus U_{2} /\left(V_{1} \cap U_{2}\right)
$$

and the bilinear form is nondegenerate on the two summands. We apply this to $V=\operatorname{Ker} L_{0}$, $V_{1}=\operatorname{Ker} L_{0} \cap H_{\leqslant-1}^{n}(X), U_{1}=\Lambda$ and $U_{2}=\eta \operatorname{Ker} L_{2}$.

We now conclude the proof of the Polarization Theorem 2.1.8 for $P_{0}^{0}$ :

Proof of Theorem 2.1.8. - Since $P_{0}^{0} \subseteq \operatorname{Ker} \eta$, we have an inclusion of Hodge structures

$$
P_{0}^{0} \subseteq\left(\eta \operatorname{Ker} L_{2}\right)^{\perp} /\left(\eta \operatorname{Ker} L_{2}\right)^{\perp} \cap H_{\leqslant-1}^{n}(X)=\Lambda_{0}
$$

which, in view of Lemma 5.4.3 and Remark 3.1.1, are polarized by $(-1)^{n} S_{00}^{\eta L}(-, C(-))$. 


\section{The Semisimplicity Theorem 2.1.1(c) for ${ }^{\mathcal{Y}} \mathcal{H}^{0}\left(f_{*} \mathbb{Q}_{X}[n]\right)$}

The set-up is as in 2.1 and we assume that 2.6.2 holds. Recall Remark 2.6.3.

In this section we prove that ${ }^{\mathcal{H}} \mathcal{H}^{0}\left(f_{*} \mathbb{Q}_{X}[n]\right)$ is semisimple, i.e. we establish the remaining case $i=0$ of Theorem 2.1.1(c) for $f$. Along the way we also prove the Generalized Grauert Contractibility Criterion 2.1.9 and the Refined Intersection Form Theorem 2.1.10, thus proving all the results in 2.1 .

\subsection{The induction on the strata: reduction to $S_{0}$}

We introduce the stratification with which we work. Let $K \in O b(D(X))$. The typical example will be $K=\mathbb{Q}_{X}[n]$. We fix once and for all $\mathfrak{X}$ and $\mathfrak{Y}$ finite algebraic Whitney stratifications for $f$ such that $K$ is $\mathfrak{X}$-cc. By 3.3 and 3.6, $f_{*} K$ and all of its perverse cohomology complexes $p_{\mathcal{H}}^{j}\left(f_{*} K\right), \forall j \in \mathbb{Z}$, are $\mathfrak{Y}$-cc.

We employ the notation in 3.2. Let $0 \leqslant s \leqslant d$. Denote by

$$
S_{s} \stackrel{\alpha_{s}}{\longrightarrow} U_{s} \stackrel{\beta_{s}}{\longleftarrow} U_{s+1}
$$

the corresponding closed and open embeddings.

The stratification $\mathfrak{Y}$ induces a stratification $\mathfrak{Y}_{U_{s}}$ on $U_{s}$ and the trivial one, $\mathfrak{Y}_{S_{s}}$, on $S_{s}$. The maps $\alpha_{s}$ and $\beta_{s}$ are stratified with respect to these stratifications.

Let $K^{\prime}$ be $\mathfrak{Y}$-cc, e.g. $K^{\prime}=f_{*} K$ or $K^{\prime}={ }^{p} \mathcal{H}^{j}\left(f_{*} K\right), l \in \mathbb{Z}$. Then $\alpha_{s}^{*} K^{\prime}$ is $\mathfrak{Y}_{S_{s}}$-cc and $\beta_{s}^{*} K^{\prime}$ is $\mathfrak{Y}_{U_{s+1}}$-cc.

Let $K^{\prime} \in O b\left(D\left(U_{s}\right)\right)$ be $\mathfrak{Y}_{U_{s}}$-cc. By 3.3, all terms of the triangle $\alpha_{s !} \alpha_{s}^{!} K^{\prime} \rightarrow K^{\prime} \rightarrow$ $\beta_{s *} \beta_{s}^{*} K^{\prime} \stackrel{[1]}{\longrightarrow}$ are $\mathfrak{Y}_{U_{s}}$-cc and the maps induced at the level of cohomology sheaves are, when restricted to the strata $S_{l}, l \geqslant s$, maps of local systems.

Let $n:=\operatorname{dim} X, m:=\operatorname{dim} f(X)$. The stratum $S_{m}$ has a unique connected component $S_{f}$ contained in the open subset of $f(X)$ over which $f$ is smooth.

Clearly, all the complexes we shall be interested in have support contained in $f(X)$. In addition, depending on whether they are defined on $Y, U_{s}$ or $S_{s}$, they are either $\mathfrak{Y}$-cc, $\mathfrak{Y}_{U_{s}}$ cc, or $\mathfrak{Y}_{S_{s}}$-cc.

Remark 6.1.1. - By the condition of (co)support in 3.6, we have

$$
{ }^{p} \mathcal{H}^{j}\left(f_{*} \mathbb{Q}_{X}[n]\right)_{\mid U_{s}} \simeq \tau_{\geqslant-m} \tau_{\leqslant-s}{ }^{p} \mathcal{H}^{j}\left(f_{*} \mathbb{Q}_{X}[n]\right)_{\mid U_{s}}, \quad \forall 0 \leqslant s \leqslant m .
$$

The sheaf $\mathcal{H}^{-s}\left({ }^{p} \mathcal{H}^{j}\left(f_{*} \mathbb{Q}_{X}[n]\right)_{\mid U_{s}}\right)$ is a local system on $S_{s}$.

Let $f_{s}: U_{s}^{\prime}:=f^{-1}\left(U_{s}\right) \rightarrow U_{s}$ be the corresponding maps. Note that $U_{s}^{\prime}=\emptyset, \forall s>m$. We have natural restriction isomorphisms

$$
{ }^{p} \mathcal{H}^{j}\left(f_{*} \mathbb{Q}_{X}[n]_{\mid U_{s}}\right) \simeq{ }^{p} \mathcal{H}^{j}\left(f_{s_{*}} \mathbb{Q}_{U_{s}^{\prime}}[n]\right) .
$$

Recall that, if $P \in \operatorname{Perv}\left(U_{s+1}\right)$, then $\beta_{s ! *} P \simeq \tau_{\leqslant-s-1} P \in \operatorname{Perv}\left(U_{s}\right)$ (cf. 3.8.1).

In this set-up, Deligne's Theorem [9] can be re-formulated in terms of the existence of an isomorphism

$$
f_{m_{*}} \mathbb{Q}_{U_{m}^{\prime}}[n] \simeq \bigoplus_{j}{ }^{\mathcal{H}} \mathcal{H}^{j}\left(f_{m_{*}} \mathbb{Q}_{U_{m}^{\prime}}[n]\right)[-j]
$$

where ${ }^{p} \mathcal{H}^{j}\left(f_{m_{*}} \mathbb{Q}_{U_{m}^{\prime}}[n]\right)$ is supported, as a complex on $U_{m}$, precisely on $S_{f}$ and is there isomorphic to $\left(R^{n-m+j} f_{m_{*}} \mathbb{Q}_{f^{-1}\left(S_{f}\right)}\right)[m]$. 
Remark 6.1.2. - The local systems $R^{n-m+j} f_{m_{*}} \mathbb{Q}_{U_{m}^{\prime}}$ on $S_{f}$ are semisimple by Deligne Semisimplicity Theorem 3.1.4. In particular, the complexes ${ }^{p} \mathcal{H}^{j}\left(f_{m_{*}} \mathbb{Q}_{U_{m}^{\prime}}[n]\right)$ are semisimple in $\operatorname{Perv}\left(U_{m}\right)$.

The following Lemma essentially reduces the proof of the missing part of the Decomposition Theorem to the local criterion of Lemma 6.1.3(b). To prove that the local criterion is met we reduce it to a global property of projective maps, Lemma 5.4.3.

LEMMA 6.1.3.-

(a) For every $(j, s) \neq(0,0)$ we have a canonical isomorphism in Perv $\left(U_{s}\right)$ :

$$
{ }^{p} \mathcal{H}^{j}\left(f_{*} \mathbb{Q}_{X}[n]\right)_{\mid U_{s}} \simeq \beta_{s * !}\left({ }^{p} \mathcal{H}^{j}\left(f_{*} \mathbb{Q}_{X}[n]\right)_{\mid U_{s+1}}\right) \oplus \mathcal{H}^{-s}\left({ }^{p} \mathcal{H}^{j}\left(f_{*} \mathbb{Q}_{X}[n]\right)_{\mid U_{s}}\right)[s],
$$

where the projection to the first summand is the (unique) lifting of truncation and the projection to the second stems from truncation (cf. 6.1.1).

(b) $\operatorname{For}(j, s)=(0,0)$

$$
{ }^{p} \mathcal{H}^{0}\left(f_{*} \mathbb{Q}_{X}[n]\right) \simeq \beta_{0 * !}\left({ }^{p} \mathcal{H}^{0}\left(f_{*} \mathbb{Q}_{X}[n]\right)_{\mid U_{1}}\right) \oplus \mathcal{H}^{0}\left({ }^{p} \mathcal{H}^{0}\left(f_{*} \mathbb{Q}_{X}[n]\right)\right)[0]
$$

if and only if the natural map of dual skyscraper sheaves (cf. 3.5.2)

$$
\mathcal{H}^{0}\left(\alpha_{0 !} \alpha_{0}{ }^{!} \mathcal{H}^{0}\left(f_{*} \mathbb{Q}_{X}[n]\right)\right) \rightarrow \alpha_{0 *} \alpha_{0}{ }^{*} \mathcal{H}^{0}\left({ }^{p} \mathcal{H}^{0}\left(f_{*} \mathbb{Q}_{X}[n]\right)\right)
$$

is an isomorphism.

Proof. - (a) The perverse sheaf ${ }^{p} \mathcal{H}^{j}\left(f_{*} \mathbb{Q}_{X}[n]\right)$ is semisimple for $j \neq 0$ by Theorem 2.1.1(c) for $f$. We apply the Splitting Criterion 4.1.3 whose hypotheses are met in view of Remark 4.1.2.

Let $j=0 .{ }^{p} \mathcal{H}^{0}\left(f_{*} \mathbb{Q}_{X}[n]\right)$ is self-dual by Poincaré-Verdier duality. By Remark 4.1.2, it is enough to check that the Splitting Criterion 4.1 .3 holds for $1 \leqslant s \leqslant m$. In the case $s=m$, ${ }^{p} \mathcal{H}^{0}\left(f_{*} \mathbb{Q}_{X}[n]\right)_{\mid U_{m}}$ is a shifted local system and there is nothing to prove. Let $1 \leqslant s \leqslant m-1$. Let $Y_{s} \subseteq Y$ be the complete intersection of $s$ hyperplane sections chosen so that (1) it meets every connected component of the pure and positive dimensional $S$ transversally at a finite set $T$ and (2) $X_{s}:=f^{-1}\left(Y_{s}\right)$ is a nonsingular variety (cf. 3.2.2). We obtain a projective morphism $f_{s}: X_{s} \rightarrow Y_{s}$. We have $\operatorname{dim} X>\operatorname{dim} X_{s}$ and we can apply our inductive hypotheses: Theorem 2.1.1(b) and (c) hold and ${ }^{p} \mathcal{H}^{0}\left(f_{s_{*}} \mathbb{Q}_{X_{s}}[n-s]\right)$ is semisimple. By Lemma 4.3.8: ${ }^{p} \mathcal{H}^{0}\left(f_{s_{*}} \mathbb{Q}_{X_{s}}[n-s]\right) \simeq{ }^{p} \mathcal{H}^{0}\left(f_{*} \mathbb{Q}_{X}[n]\right)_{\mid Y_{s}}[-s]$.

The semisimplicity of ${ }^{m} \mathcal{H}^{0}\left(f_{s_{*}} \mathbb{Q}_{X_{s}}[n-s]\right)$ implies, via Remark 4.1.2, that the conditions for the splitting criterion for ${ }^{\eta} \mathcal{H}^{0}\left(f_{s *} \mathbb{Q}_{X_{s}}[n-s]\right)$ of Lemma 4.1.3 are met at every point of $T$ which is a subset of the set of zero-dimensional strata for $f_{s}$.

By the second part of Lemma 3.5.3, we have that the splitting condition for ${ }^{p} \mathcal{H}^{0}\left(f_{*} \mathbb{Q}_{X}[n]\right)_{\mid U_{s}}$ is met as well.

(b) Since we have the result for $U_{1}$, the statement is a mere re-formulation of Lemma 4.1.3.

\subsection{The local system $\mathcal{H}^{-s}\left(\alpha_{s}^{!} p \mathcal{H}^{0}\left(f_{*} \mathbb{Q}_{X}[n]\right)\right)$ on $S_{s}$}

LEMMA 6.2.1. - Let $Z$ be an affine algebraic variety, $Q \in D^{\leqslant 0}(Z)$, i.e. $\operatorname{dim} \operatorname{supp}\left(\mathcal{H}^{i}(Q)\right) \leqslant$ $-i$. Let $\alpha: \Sigma \rightarrow Y$ be the closed embedding of the possibly empty support of $\mathcal{H}^{0}(Q)$.

Then the natural restriction map below is surjective

$$
\mathbb{H}^{0}(Z, Q) \rightarrow \mathbb{H}^{0}\left(Z, \alpha_{*} \alpha^{*} Q\right) .
$$

$4^{\text {e }}$ SÉRIE - TOME $38-2005-\mathrm{N}^{\circ} 5$ 
Proof. - We have the two spectral sequences $E_{2}^{p q}(Q)=\mathbb{H}^{p}\left(Z, \mathcal{H}^{q}(Q)\right) \Longrightarrow \mathbb{H}^{p+q}(Z, Q)$, $E_{2}^{p q}\left(\alpha_{*} \alpha^{*} Q\right)=\mathbb{H}^{p}\left(Z, \mathcal{H}^{q}\left(\alpha_{*} \alpha^{*} Q\right)\right) \Rightarrow \mathbb{H}^{p+q}\left(Z, \alpha_{*} \alpha^{*} Q\right)$. The natural adjunction map $a: Q \rightarrow$ $\alpha_{*} \alpha^{*} Q$ induces a map of spectral sequences $E_{r}(Q) \rightarrow E_{r}\left(\alpha_{*} \alpha^{*} Q\right)$.

Note that $\operatorname{dim} \Sigma \leqslant 0$. The assumptions on $Q$ imply that $E_{2}^{p q}\left(\alpha_{*} \alpha^{*} Q\right)=0$ if either $p \neq 0$, or $q>0$ so that $E_{2}\left(\alpha_{*} \alpha^{*} Q\right)=E_{\infty}\left(\alpha_{*} \alpha^{*} Q\right)$. In particular, $\mathbb{H}^{0}\left(Z, \alpha_{*} \alpha^{*} Q\right)=E_{\infty}^{00}\left(\alpha_{*} \alpha^{*} Q\right)=$ $E_{2}^{00}\left(\alpha_{*} \alpha^{*} Q\right)$.

Since $Q \in D^{\leqslant 0}(Z), Q$ is $\mathfrak{Z}$-cc with respect to some stratification $\mathfrak{Z}$ of $Z$, $\operatorname{supp} \mathcal{H}^{q}(Q)$ is a closed affine subset of $Z$ of dimension at most $-q$. The theorem on the cohomological dimension of affine sets with respect to constructible sheaves, [21, Theorem 10.3.8], implies that $E_{2}^{p q}(Q)=0$ for every $p+q>0$.

We have $E_{2}^{p q}(Q)=E_{\infty}^{p q}(Q)=0$ if either $p<0$ or $p+q>0$.

It follows that we have the surjection

$$
\mathbb{H}^{0}(Z, Q) \rightarrow E_{\infty}^{00}(Q)=E_{\infty}^{00}\left(\alpha_{*} \alpha^{*} Q\right)=\mathbb{H}^{0}\left(Z, \alpha_{*} \alpha^{*} Q\right)
$$

In what follows, by the conditions of support for perverse sheaves, supp $\mathcal{H}^{0}\left({ }^{p} \mathcal{H}^{b}\left(f_{*} \mathbb{Q}_{X}[n]\right)\right)$ is either empty, or a finite set of points. In the first case, Proposition 6.2.2 is trivial.

Proposition 6.2.2. - Let $b \in \mathbb{Z}$ and $\alpha$ be the closed embedding into $X$ of the zero-dimensional set $\operatorname{supp} \mathcal{H}^{0}\left({ }^{p} \mathcal{H}^{b}\left(f_{*} \mathbb{Q}_{X}[n]\right)\right)=\left\{y_{1}, \ldots, y_{r}\right\}$. The restriction map

$$
H_{b}^{n+b}(X)=\mathbb{H}^{0}\left(Y,{ }^{p} \mathcal{H}^{b}\left(f_{*} \mathbb{Q}_{X}[n]\right)\right) \rightarrow \mathbb{H}^{0}\left(Y, \alpha_{*} \alpha^{*} \mathcal{H}^{b}\left(f_{*} \mathbb{Q}_{X}[n]\right)\right)=\bigoplus_{i=1, \ldots, r} H_{b}^{n+b}\left(f^{-1}\left(y_{i}\right)\right)
$$

is surjective. Dually, the cycle map below is injective

$$
\bigoplus_{i=1, \ldots, r} H_{n+b,-b}^{\mathrm{BM}}\left(f^{-1}\left(y_{i}\right)\right) \rightarrow H_{-b}^{n-b}(X) .
$$

Proof. - Let $U \subseteq Y$ be an affine open set such that $\operatorname{supp} \mathcal{H}^{0}\left({ }^{p} \mathcal{H}^{b}\left(f_{*} \mathbb{Q}_{X}[n]\right)\right) \subseteq U$ and $U^{\prime}:=f^{-1}(U)$. Consider the commutative diagram

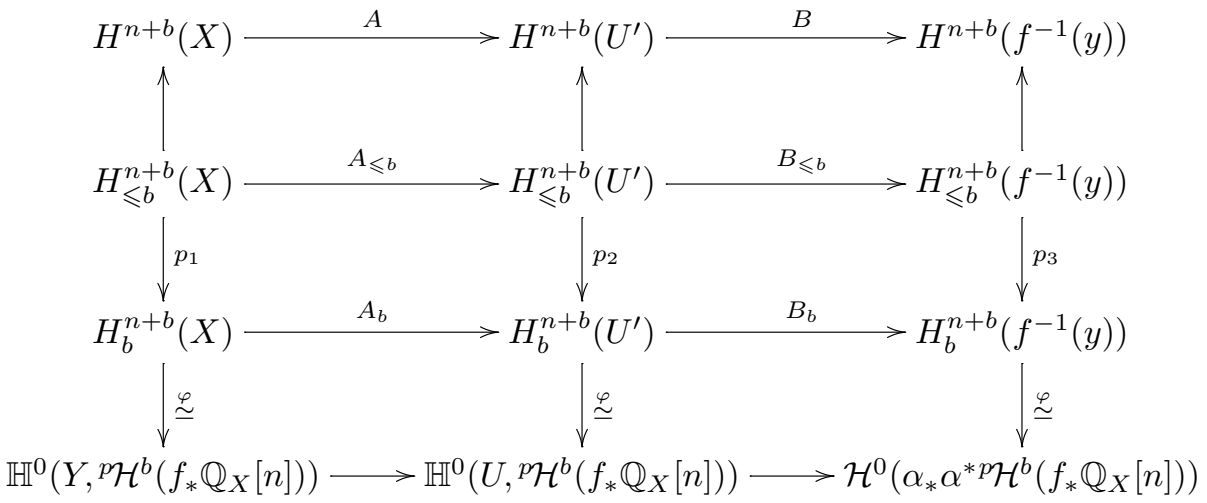

where the vertical maps pointing up are the natural injections, the quotient maps $p_{i}$ are surjective and the vertical maps on the bottom row are the identifications of Remark 4.3.4. In view of the existing splitting $\varphi$, the maps $A$ and $B$ are strict with respect to the perverse filtrations on $H^{n+b}(X), H^{n+b}(U)$ and the induced filtration on $H^{n+b}\left(f^{-1}(y)\right)$ (cf. 4.3.6). 
By Lemma 6.2.1, $B_{b}$ is surjective. This implies that $B_{b} \circ p_{2}=p_{3} \circ B_{\leqslant b}$ is surjective.

By Deligne's Theory of Mixed Hodge Structures, [12, Proposition 8.2.6], $\operatorname{Im} B \circ A=\operatorname{Im} B$. By the strictness with respect to the perverse and to the induced filtration, we infer that $\operatorname{Im} B_{\leqslant b} \circ A_{\leqslant b}=\operatorname{Im} B_{\leqslant b}$

It follows that $p_{3} \circ B_{\leqslant b} \circ A_{\leqslant b}=\left(B_{b} \circ A_{b}\right) \circ p_{1}$ is surjective and so is $B_{b} \circ A_{b}$, i.e. we have proved the wanted surjectivity.

\section{3. ${ }^{\eta} \mathcal{H}^{0}\left(f_{*} \mathbb{Q}_{X}[n]\right)$ is a direct sum of intersection cohomology complexes}

In this section we prove Proposition 6.3.2, i.e. an important step towards the semisimplicity of ${ }^{\gamma} \mathcal{H}^{0}\left(f_{*} \mathbb{Q}_{X}[n]\right)$. A key ingredient is the Generalized Grauert Contractibility Criterion 2.1.9, which is concerned with the Hodge-theoretic properties of the refined intersection form $H_{n-b, b}^{\mathrm{BM}}\left(f^{-1}(y)\right) \rightarrow H_{b}^{n+b}\left(f^{-1}(y)\right)$ introduced in 3.4. Together with Lemma 4.3.6, Theorem 2.1.9 gives complete information on the structure of the refined intersection form on the fibers of $f$.

Proof of the Generalized Grauert Contractibility Criterion 2.1.9. - The injectivity follows from the second statement in Proposition 6.2.2. If $y \notin \operatorname{supp} \mathcal{H}^{0}\left({ }^{p} \mathcal{H}^{b}\left(f_{*} \mathbb{Q}_{X}[n]\right)\right)$, then $H_{n-b, b}^{\mathrm{BM}}\left(f^{-1}(y)\right)=0$ and the injectivity statement is trivial.

The inclusion in $\operatorname{Ker} L$ follows from the fact that one can find a hyperplane section of $Y$ avoiding $y$.

The class map $c l: H_{n-b}^{\mathrm{BM}}\left(f^{-1}(y)\right) \rightarrow H^{n+b}(X)$ is a map of mixed Hodge structures so that the image $\operatorname{Im}(c l) \subseteq H^{n+b}(X)$ is a pure Hodge sub-structure. By Lemma 4.3.6, the class map is filtered and $H_{n-b}^{\mathrm{BM}}\left(f^{-1}(y)\right)=H_{n-b, \leqslant b}^{\mathrm{BM}}\left(f^{-1}(y)\right)$.

It follows that the projection, $\operatorname{Im}\left(c l_{b}\right)$, of $\operatorname{Im}(c l)$ to $H_{b}^{n+b}(X)$ is a pure Hodge sub-structure.

The compatibility with the direct sum decomposition given by Theorem 2.1.1(a) and 4.4.4 follows from the additivity of $\alpha ! \alpha^{!}$, where $\alpha: y \rightarrow Y$.

By the Generalized Hodge-Riemann Bilinear Relations 2.1.8, the direct summands of $\operatorname{Im}\left(c l_{b}\right)$ are $S_{-b 0}^{\eta L}$-orthogonal. Since $\operatorname{Im}\left(c l_{b}\right)$ is a Hodge sub-structure of $H_{b}^{n+b}(X)$, the form $S_{-b 0}^{\eta L}$ induces a polarization on each direct summand (cf. 3.1.1).

The proof of Proposition 6.3.2 requires only the case $b=0$ of Theorem 2.1.9. Consider the natural adjunction map

$$
A: \alpha_{!} \alpha^{!} \mathcal{H}^{0}\left(f_{*} \mathbb{Q}_{X}[n]\right) \rightarrow{ }^{p} \mathcal{H}^{0}\left(f_{*} \mathbb{Q}_{X}[n]\right) .
$$

Proposition 6.3.1. - The map

$$
\mathcal{H}^{0}(A)_{y}: \mathcal{H}^{0}\left(\alpha_{!} \alpha^{!} \mathcal{H}^{0}\left(f_{*} \mathbb{Q}_{X}[n]\right)\right)_{y} \rightarrow \mathcal{H}^{0}\left({ }^{p} \mathcal{H}^{0}\left(f_{*} \mathbb{Q}_{X}[n]\right)\right)_{y}
$$

is an isomorphism.

Proof. - Since the domain and the target have the same rank, it is enough to show injectivity. Let

$$
A^{\prime}: \alpha_{!} \alpha^{! p} \mathcal{H}^{0}\left(f_{*} \omega_{X}[-n]\right) \rightarrow{ }^{p} \mathcal{H}^{0}\left(f_{*} \omega_{X}[-n]\right)
$$

be the natural adjunction map. In view of Remark 3.4.2, the statement to be proved is equivalent to the analogous statement for the map $\mathcal{H}^{0}\left(A^{\prime}\right)_{y}$. Consider the composition

$$
I: \alpha_{!} \alpha^{!}{ }^{p} \mathcal{H}^{0}\left(f_{*} \omega_{X}[-n]\right) \rightarrow{ }^{p} \mathcal{H}^{0}\left(f_{*} \omega_{X}[-n]\right) \simeq{ }^{p} \mathcal{H}^{0}\left(f_{*} \mathbb{Q}_{X}[n]\right) \rightarrow \alpha_{*} \alpha^{* p} \mathcal{H}^{0}\left(f_{*} \mathbb{Q}_{X}[n]\right) .
$$

$4^{\mathrm{e}}$ SÉRIE - TOME $38-2005-\mathrm{N}^{\circ} 5$ 
By the self-duality of $I$, the domain and target of $\mathcal{H}^{0}\left(A^{\prime}\right)_{y}$ have the same rank. The linear map $\mathcal{H}^{0}(I)_{y}$ is the refined intersection form $H_{n, 0}^{\mathrm{BM}}\left(f^{-1}(y) \rightarrow H_{0}^{n}\left(f^{-1}(y)\right)\right.$ which is an isomorphism by Theorem 2.1.9. This implies that $\mathcal{H}^{0}\left(A^{\prime}\right)_{y}$ is injective and hence an isomorphism.

Proposition 6.3.2. - There are canonical isomorphisms in Perv $(Y)$ for every $b$ :

$$
{ }^{p} \mathcal{H}^{b}\left(f_{*} \mathbb{Q}_{X}[n]\right) \simeq \bigoplus_{l=0}^{\operatorname{dim} Y} I C_{\overline{S_{l}}}\left(\alpha_{l}^{*} \mathcal{H}^{-l}\left({ }^{p} \mathcal{H}^{b}\left(f_{*} \mathbb{Q}_{X}[n]\right)\right)\right)
$$

Proof. - It follows from Lemma 6.1.3 and Proposition 6.3.1.

Proof of the Refined Intersection Form Theorem 2.1.10. - By Lemma 4.3.6 we only need to deal with the case $a=b$. In this case, by 4.1.3, the nondegeneracy of the refined intersection form in question is precisely the obstruction to the splitting of ${ }^{p} \mathcal{H}^{b}\left(f_{*} \mathbb{Q}_{X}[n]\right)$ so that the statement follows from Proposition 6.3.2.

\subsection{The semisimplicity of ${ }^{p} \mathcal{H}^{0}\left(f_{*} \mathbb{Q}_{X}[n]\right)$}

The goal of this section is to prove Theorem 6.4.2. The local systems in question do not seem to arise as the ones associated with the cohomology of the fibers of a smooth map, so that Deligne's semisimplicity result 3.1.4 does not apply directly. The idea of the proof is to use hyperplane sections on $Y$ to find a smooth projective family $\mathcal{X}_{T} \rightarrow T$ of $(n-s)$-dimensional varieties over a Zariski-dense open subset $T$ of $S_{s}$ in a way that allows to use Proposition 6.2.2 to infer that, over $T$, the local system $H_{0}^{n-s}\left(\mathcal{X}_{t}\right)$ maps surjectively onto the local system $H_{0}^{n-s}\left(f^{-1}(t)\right)$. The left-hand side is semisimple by Deligne's Semisimplicity Theorem 3.1.4. It follows that so is the right-hand side. On the other hand, the latter is the restriction of $\mathcal{H}^{-s}\left(\alpha_{s}^{*} \eta \mathcal{H}^{0}\left(f_{*} \mathbb{Q}_{X}[n]\right)\right)$ to $T$ and the semisimplicity over $S_{s}$ follows (cf. 3.1.3).

We need a relative version of Proposition 6.2.2.

LEMMA 6.4.1. - Let

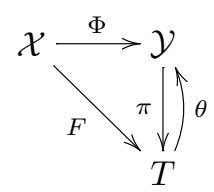

be projective maps of quasi-projective varieties such that:

(1) $\mathcal{X}$ is nonsingular of dimension $n, T$ is nonsingular of dimension $s$;

(2) $F:=\pi \circ \Phi$ is surjective and smooth of relative dimension $n-s$;

(3) the map $\Phi$ is stratified in the sense of Theorem 3.2.3 and the strata of $\mathcal{Y}$ map smoothly and surjectively onto $T$;

(4) $\theta$ is a section of $\pi$, i.e. $\pi \circ \theta=\operatorname{Id}_{T}$ and $\theta(T)$ is a stratum of $\mathcal{Y}$;

(5) there is an isomorphism $\Phi_{*} \mathbb{Q}_{\mathcal{X}}[n] \simeq \bigoplus_{l}{ }^{\mathcal{H}} \mathcal{H}^{l}\left(\Phi_{*} \mathbb{Q}_{\mathcal{X}}[n]\right)[-l]$.

Then there is a surjective map of local systems on $T$ :

$$
\sigma: R^{n-s} F_{*} \mathbb{Q}_{\mathcal{X}} \rightarrow \mathcal{H}^{-s}\left(\theta^{* p} \mathcal{H}^{0}\left(\Phi_{*} \mathbb{Q}_{\mathcal{X}}[n]\right)\right) .
$$

In particular, the local system $\mathcal{H}^{-s}\left(\theta^{* p} \mathcal{H}^{0}\left(\Phi_{*} \mathbb{Q}_{\mathcal{X}}[n]\right)\right)$ on $T$ is semisimple. 
Proof. - By assumption (3), the sheaves in question are indeed local systems on $T$. The closed embedding $i: t \rightarrow T$ of a point in $T$ induces the following diagram with Cartesian squares:

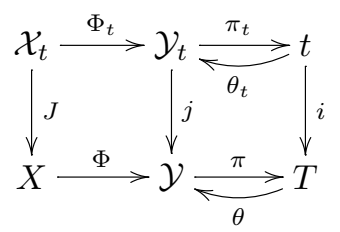

There is the commutative diagram

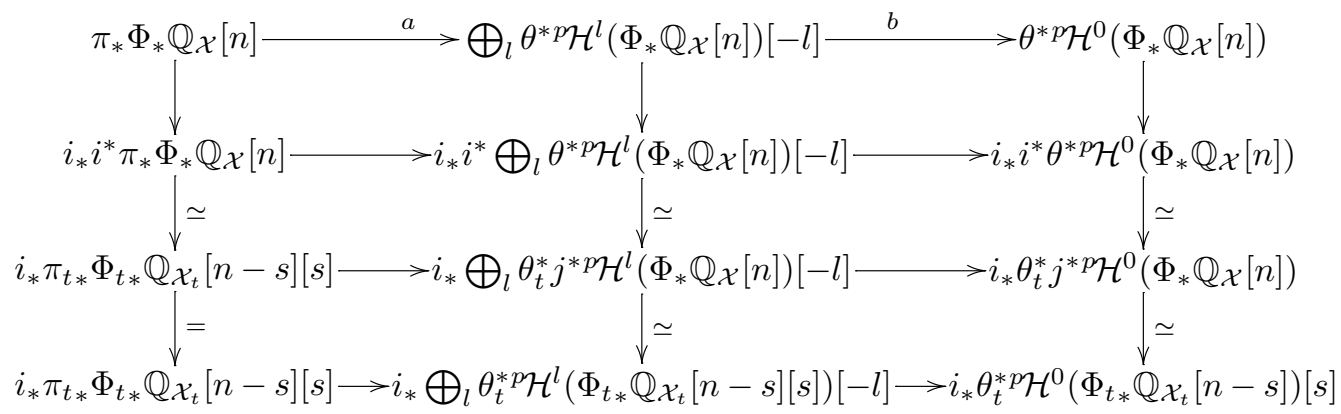

which is obtained as follows. The first row: the first map is obtained by applying $\pi_{*}$ to the adjunction map for $\theta$ and by using (4) and (5); the second map is the natural projection. The first column of maps, is the adjunction relative to $i$. The third row is obtained from the second one using the usual base change relations: $i^{*} \pi_{*} \simeq \pi_{t *} j^{*}, j^{*} \Phi_{*} \simeq \Phi_{t *} J^{*}$ and the equality $i^{*} \theta^{*}=\theta_{t}^{*} j^{*}$. The commutativity of the bottom follows from Lemma 4.3.8 in view of the fact that the codimension $s$ embedding $j: \mathcal{Y}_{t} \rightarrow \mathcal{Y}$ is transverse to all the strata of $\mathfrak{Y}$ by (3).

Keeping in mind (2), define $\sigma$ to be $\mathcal{H}^{-s}(b \circ a)$.

The map $\sigma_{t}$ at the level of stalks is identified to the analogous map on the bottom row which reads as $H^{n-s}\left(\mathcal{X}_{t}\right) \rightarrow H_{0}^{n-s}\left(\Phi_{t}^{-1}(t)\right)$ and is surjective by Proposition 6.2.2.

We conclude by the Semisimplicity Theorem 3.1.4.

The following theorem concludes the proof of the semisimplicity of ${ }^{\mathcal{H}} \mathcal{H}^{0}\left(f_{*} \mathbb{Q}_{X}[n]\right)$, for it shows that every direct summand of it is an intersection cohomology complex associated with a semisimple local system on some locally closed smooth subvariety.

THEOREM 6.4.2. - The local systems $\mathcal{H}^{-s}\left(\alpha_{s}^{* p} \mathcal{H}^{0}\left(f_{*} \mathbb{Q}_{X}[n]\right)\right)$ are semisimple.

Proof. - The statement is trivial for $s=0$ and $s>m=\operatorname{dim} f(X)$. The case $s=m$ follows by Remark 6.1.2. We may assume that $1 \leqslant s \leqslant m-1$. We shall reduce this case to Lemma 6.4.1.

In view of Remark 3.1.3, it is enough to show semisimplicity over a Zariski-dense open subset $T$ of every connected component of $S_{s}$.

Let $\mathbb{P}^{\vee}=|A| \simeq \mathbb{P}^{d^{\prime}}$ be the very ample linear system on $Y$ associated with $A, \Pi:=\left(\mathbb{P}^{\vee}\right)^{s}$, $d:=s d^{\prime}=\operatorname{dim} \Pi$. A point $p \in \Pi$ corresponds to an $s$-tuple $\left(H_{1}, \ldots, H_{s}\right)$ of hyperplanes in $\mathbb{P}$.

Consider the universal $s$-fold complete intersection families $\mathcal{Y}:=\left\{(y, p) \mid y \in \bigcap_{j=1}^{s} H_{j}\right\} \subseteq$ $Y \times \Pi$ and $\mathcal{X}:=\mathcal{Y} \times_{Y \times \Pi}(X \times \Pi) \subseteq X \times \Pi$. Note that $\mathcal{X}$ is nonsingular and the general member of the family $\mathcal{X}$ over $\Pi$ is nonsingular and connected by the Bertini Theorems; in fact 
the assumption $1 \leqslant s \leqslant m-1$ implies $\operatorname{dim} f(X) \geqslant 2$. However, the connectedness plays no essential role.

For every map $W \rightarrow Y$ there is the commutative diagram with Cartesian squares

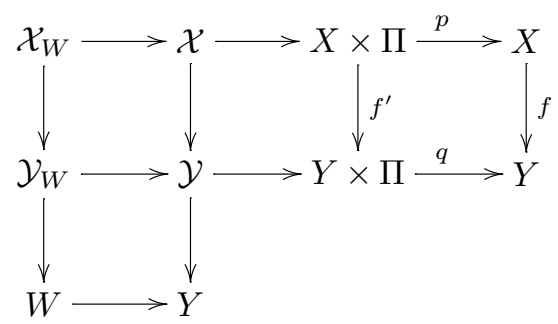

The base-point-freeness of $|A|$ implies that $\mathcal{Y}_{W} \rightarrow W$ is Zariski-locally trivial. Since the general complete intersection of $s$ hyperplanes meets the $s$-dimensional $S$ in a nonempty and finite set, the natural map

$$
b: \mathcal{Y}_{S} \rightarrow \Pi
$$

is dominant.

By Bertini Theorem for $|L|$ (cf. 3.2.2), generic smoothness for $\mathcal{X} \rightarrow \Pi$, the algebraic Thom Isotopy Lemmas (Theorem 3.2.3) and generic smoothness for $\mathcal{Y} \rightarrow \Pi$, there is a Zariski-dense open subset $\Pi^{0} \subseteq \Pi$ such that:

(1) the surjective map $\mathcal{X} \rightarrow \Pi$ is smooth over $\Pi^{0}$;

(2) the complete intersections $Y_{s}$ of $s$ elements associated with the points of $\Pi^{0}$ meet all strata of $Y$ transversally;

(3) the restriction of $h: \mathcal{Y} \rightarrow \Pi$ over $\Pi^{0}$ is stratified so that every stratum maps surjectively and smoothly to $\Pi^{0}$.

Since $b$ is dominant, $b^{-1} \Pi^{0}$ is Zariski-dense and open in $\mathcal{Y}_{S}$.

Since $\mathcal{Y}_{S} \rightarrow S$ is Zariski-locally trivial, there exists a Zariski-dense open subset $T \subseteq S$ such that $\mathcal{Y}_{T} \rightarrow T$ admits a section $\mu: T \rightarrow \mathcal{Y}_{T}$ with the property that $\mu(T) \subseteq b^{-1} \Pi^{0}$.

By shrinking $T$, we may assume that the quasi-finite map $b \circ \mu: T \rightarrow b(\mu(T)) \subseteq \Pi^{0} \subseteq \Pi$ is smooth, of relative dimension zero.

We have a commutative diagram with Cartesian squares

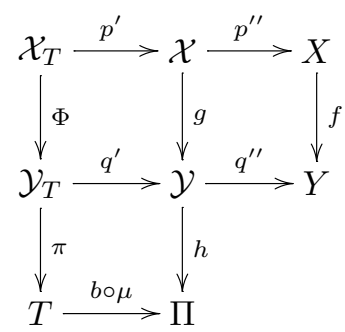

The map $\Phi$ inherits a stratification from the one on $g$ by pull-back and all strata on $\mathcal{Y}_{T}$ map surjectively and smoothly onto $T$.

For every $t \in T, Y_{t}:=\pi^{-1}(t)$ is a complete intersection of $s$ hyperplanes passing through $t \in T \subseteq Y$, meeting all the strata of $Y$ transversally and such that $X_{t}:=(\pi \circ \Phi)^{-1}(t)$ is a smooth projective variety of dimension $n-s$. Note that the map $\pi$ has a tautological section $\theta: T \rightarrow \mathcal{Y}_{T}$ assigning to $t \in T$ the same point $t \in \mathcal{Y}_{t}$. 
We have a commutative diagram where the upper square is Cartesian:

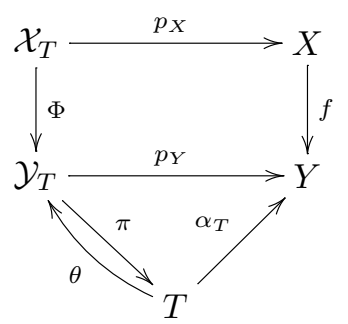

We have proved Theorem 2.1.1(b) for $f$ so that $f_{*} \mathbb{Q}_{X}[n] \simeq \bigoplus_{l}{ }^{p} \mathcal{H}^{l}\left(f_{*} \mathbb{Q}_{X}[n]\right)[-l]$. By Lemma 4.3.8 and Remark 4.3.9, we have, via pull-back, analogous decompositions for $g$ and for $\Phi$ and an isomorphism $\left.\left.p_{Y}^{*} \mathcal{H}^{0}\left(f_{*} \mathbb{Q}_{X}[n]\right)\right) \simeq{ }^{p} \mathcal{H}^{0}\left(\Phi_{*} \mathbb{Q}_{\mathcal{X}_{T}}[n]\right)\right)$.

We are now in the position to apply Lemma 6.4.1 to the diagram $\mathcal{X}_{T} \stackrel{\Phi}{\longrightarrow} \mathcal{Y}_{T} \stackrel{\pi}{\longrightarrow} T \stackrel{\theta}{\longrightarrow} \mathcal{Y}_{T}$ and deduce the semisimplicity of

$$
\mathcal{H}^{-s}\left(\theta^{* p} \mathcal{H}^{0}\left(\Phi_{*} \mathbb{Q}_{\mathcal{X}_{T}}[n]\right)\right) \simeq \mathcal{H}^{-s}\left(\theta^{*} p_{Y}^{*} \mathcal{H}^{0}\left(f_{*} \mathbb{Q}_{X}[n]\right)\right) \simeq \mathcal{H}^{-s}\left(\alpha_{T}^{*} \mathcal{H}^{0}\left(f_{*} \mathbb{Q}_{X}[n]\right)\right) .
$$

We conclude by 3.1.3 applied to $T \subseteq S$.

\section{The pure Hodge structure on Intersection Cohomology}

In this section we prove the Purity Theorem 2.2.1 and the Hodge-Lefschetz Theorem for Intersection Cohomology 2.2.3.

\subsection{The Purity Theorem}

Note that if $\operatorname{dim} X=1$, then Theorem 2.2.1 holds trivially.

LEMMA 7.1.1. - If Theorem 2.2.1 holds for every map $g: Z \rightarrow Z^{\prime}$ of projective varieties, $Z$ nonsingular, $\operatorname{dim} Z<\operatorname{dim} X$, then it holds for every group $H_{i}^{j}(X)(i, j) \neq(0, n)$.

Proof. - Fix $i<0$. Let $r: X^{1} \rightarrow X$ be a nonsingular hyperplane section. Choose stratifications for $f$ and $g$ which have in common the stratification of $Y$.

By the Weak-Lefschetz-type Proposition 4.7.6(i), the Semisimplicity Theorem 2.1.1(c) and by the Hodge Structure Theorem 2.1.5 coupled with Remark 4.2.3, the restriction map $r^{*}: H_{i}^{j}(X) \rightarrow H_{i+1}^{j}\left(X^{1}\right)$ is an injective map of pure Hodge structures.

By Remark 3.8.2, the restriction map $r^{*}$ is a direct sum map

$$
r^{*}=\sum r_{l, S}^{*}: \bigoplus_{l, S} H_{i, l, S}^{j}(X) \rightarrow \bigoplus_{l, S} H_{i+1, l, S}^{j}\left(X^{1}\right) .
$$

Let $\widetilde{S}$ be a connected component of a stratum $S_{\tilde{l}}$. The inductive hypothesis holds for the map $g:=f \circ r: X^{1} \rightarrow Y$. This implies that the natural projection map

$$
\pi: \bigoplus_{l, S} H_{i+1, l, S}^{j}\left(X^{1}\right) \rightarrow \bigoplus_{l, S \neq \tilde{S}} H_{i+1, l, S}^{j}\left(X^{1}\right)
$$

is a map of pure Hodge structures and so is the composition $\pi \circ r^{*}$.

$4^{\mathrm{e}}$ SÉRIE - TOME $38-2005-\mathrm{N}^{\circ} 5$ 
Clearly, $H_{i, \tilde{l}, \tilde{S}}^{j}(X)=\operatorname{Ker}\left(\pi \circ r^{*}\right)$ is then a Hodge sub-structure for every $j \geqslant 0$ and every $i<0$.

The same argument as above, using Proposition 4.7.6(ii) and a cokernel instead of a kernel, shows that any $H_{i, \tilde{l}, \tilde{S}}^{j}(X)$ is a Hodge sub-structure for every $j \geqslant 0$ and every $i>0$.

Let $i=0$. There are two cases left: $j<n$ and $j>n$. They are handled in the same way as above, by first replacing $X^{1}$ with $X_{1}=f^{-1}\left(Y_{1}\right)$ the pre-image of a general hyperplane section on $Y$, and then by using Proposition 4.7.7 instead of Proposition 4.7.6.

Recall that the bilinear form $S_{00}^{\eta L}$ on $H_{0}^{n}(X)$ is induced by the Poincaré pairing $\int_{X}-\wedge-$ on $X$; see 5.2.4, (30) and (34).

LEMma 7.1.2. - The direct sum decomposition $H_{0}^{n}(X)=\bigoplus_{S} H_{0, l, S}^{n}(X)$ is $S_{00}^{\eta L}$-orthogonal.

Proof. - The duality isomorphism $\varepsilon: \mathbb{Q}_{X}[n] \simeq \omega_{X}[-n]$ induces the isomorphism

$$
p_{\mathcal{H}}^{0}\left(f_{*} \mathbb{Q}_{X}[n]\right) \stackrel{{ }^{p} \mathcal{H}^{0}(\varepsilon)}{\simeq} \mathcal{D}\left({ }^{p} \mathcal{H}^{0}\left(f_{*} \mathbb{Q}_{X}[n]\right)\right)
$$

giving $S_{00}^{\eta L}$ in hypercohomology. Setting $L_{0, l, S}:=L_{0, l \mid S}$, this gives rise to an isomorphism

$$
\bigoplus_{0, l, S} I C_{\bar{S}}\left(L_{0, l, S)} \simeq \bigoplus_{0, l, S} I C_{\bar{S}}\left(L_{0, l, S}^{\vee}\right)\right.
$$

which is a direct sum map by Remark 3.8.2.

LEMMA 7.1.3. - Let $V$ be a pure Hodge structure of weight $n . \Psi: V \otimes V \rightarrow \mathbb{Q}(-n)$ be a map of pure Hodge structures which is nondegenerate as a bilinear form. Assume that $V=V_{1} \oplus V_{2}$ with $V_{1} \subseteq V$ a pure Hodge sub-structure and that $V_{1} \perp_{\Psi} V_{2}$.

Then $V_{2} \subseteq V$ is a pure Hodge sub-structure.

Proof. - The space $V_{2}$ is the kernel of the composition $V \rightarrow V^{\vee} \rightarrow V_{1}^{\vee}$.

Proof of the Purity Theorem 2.2.1. - The proof is by induction on $\operatorname{dim} X$.

The statement is trivial when $\operatorname{dim} X=1$, for $f(X)$ is either a point or another curve and in either case there is only one direct summand.

Assume that we have proved the statement for every map $g: Z \rightarrow Z^{\prime}$ of projective varieties with $Z$ nonsingular and $\operatorname{dim} Z<\operatorname{dim} X$.

By Lemma 7.1.1 we are left with the case of $H_{0}^{n}(X)$.

Fix a connected component $S$ of a non-dense stratum $S_{l}$. Let $L_{S}:=L_{0, l_{\mid S}}$ and $y \in S$.

CLAIM. - $L_{S, y}=H_{0}^{n-l}\left(f^{-1}(y)\right)=H_{\leqslant 0}^{n-l}\left(f^{-1}(y)\right)$ is a weight $(n-l)$ pure Hodge substructure of the mixed Hodge structure $H^{n-l}\left(f^{-1}(y)\right)$.

Proof. - The first equality is the definition of $L_{S}$ (see Proposition 6.3.2). Let $Y_{l} \subseteq$ be the intersection of $l$ sufficiently general hyperplane sections of $Y$ through $y \in S$ and $f_{l}: X_{l}:=f^{-1}\left(Y_{l}\right) \rightarrow$ $Y_{l}$ be the resulting map. Clearly, $f_{l}^{-1}(y)=f^{-1}(y)$. The filtrations on the cohomology group $H^{n-l}\left(f^{-1}(y)\right)$ induced by the two maps $f_{l}$ and $f$ coincide by Lemma 4.3.8. The second equality follows from Lemma 4.3.6 applied to $f_{l}$. Theorem 2.1.9, applied to $f_{l}$, gives the last statement of the claim.

Let

$$
\rho: Z_{S} \rightarrow Z_{S}^{\prime}:=\overline{f^{-1}(S)}
$$


be a proper surjective map, with $Z_{S}$ nonsingular and projective and of dimension $\operatorname{dim} Z_{S}=$ $\operatorname{dim} Z_{S}^{\prime}<n$. For example, a resolution of the singularities of the irreducible components of $Z_{S}^{\prime}$. Note that $Z_{S}$ is not necessarily pure-dimensional.

Let $S_{s m} \subseteq S$ be the Zariski-dense open set over which $f \circ \rho$ is smooth.

By refining the stratification, we may assume that $S_{s m}=S$. In fact, the new strata in $S \backslash S_{s m}$ will not contribute any new direct summand to $H_{0}^{n}(X)$.

Setting $g:=f \circ \rho: Z_{S} \rightarrow Y$, the map

$$
\rho: g^{-1}(y) \rightarrow f^{-1}(y)
$$

is proper and surjective from a nonsingular space.

By Theorem 3.1.6, the map $H^{n-l}\left(f^{-1}(y)\right) \stackrel{\rho^{*}}{\rightarrow} H^{n-l}\left(g^{-1}(y)\right)$ is such that

$$
\operatorname{Ker} \rho^{*}=W_{n-l-1} H^{n-l}\left(f^{-1}(y)\right) .
$$

Since $H_{\leqslant 0}^{n-l}\left(f^{-1}(y)\right)$ is of pure weight $n-l$,

$$
\operatorname{Ker} \rho^{*} \cap H_{\leqslant 0}^{n-l}\left(f^{-1}(y)\right)=\{0\}
$$

so that

$$
\rho_{\mid}^{*}: H_{\leqslant 0}^{n-l}\left(f^{-1}(y)\right) \rightarrow H^{n-l}\left(g^{-1}(y)\right)
$$

is injective. It follows that so is the map of local systems

$$
L_{S} \rightarrow\left(R^{n-l} g_{*} \mathbb{Q}_{Z_{S}}\right)_{\mid S} .
$$

By Deligne's Semisimplicity Theorem 3.1.4, this injection splits. Let

$$
Z_{S}=\coprod_{t \geqslant 0} Z_{S}^{t}
$$

be the decomposition into pure-dimensional "components".

By Remark 4.2.3 and the Hodge Structure Theorem 2.1.5, we get that the natural pull-back is a map of pure Hodge structures:

$$
\rho^{*}: H_{0}^{n}(X) \rightarrow \bigoplus_{0 \leqslant t \leqslant n-1} H_{n-t}^{n}\left(Z_{S}^{t}\right)
$$

Denote by $\pi: \bigoplus_{0 \leqslant t \leqslant n-1} H_{n-t}^{n}\left(Z_{S}^{t}\right) \rightarrow V$ the projection corresponding to the direct summand associated with $\left(R^{n-s} g_{*} \mathbb{Q}_{Z_{S}}\right)_{\mid S}$.

Since $t<n$, we can apply the inductive hypothesis and $\pi$ is a map of pure Hodge structures.

By (44),

$$
\operatorname{Ker}\left(\pi \circ \rho^{*}\right)=\bigoplus_{l^{\prime}, S^{\prime} \neq S} H_{0, l^{\prime}, S^{\prime}}^{n}(X) \subseteq H_{0}^{n}(X)
$$

is a Hodge sub-structure.

$4^{\mathrm{e}}$ SÉRIE - TOME $38-2005-\mathrm{N}^{\circ} 5$ 
By Lemmas 7.1.2 and 7.1.3, we have that

$$
H_{0, l, S}^{n}(X) \subseteq H_{0}^{n}(X)
$$

is a pure Hodge sub-structure for every non-dense stratum $S$. This implies immediately that so is any direct sum over non-dense strata.

Applying the two lemmata again, we conclude that the contribution from the dense stratum is also a pure Hodge sub-structure.

\subsection{The Hodge-Lefschetz Theorem}

We need the following

LEMMA 7.2.1. - Let

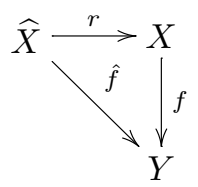

be such that $\hat{f}$ and $f$ are resolutions and $r$ is proper and surjective. Let $Y=\amalg S_{l}$ be a stratification of $Y$ part of stratifications for $\hat{f}$ and for $f$. Let $y \in S$ be the choice of a point on a connected component of a stratum. We have the diagram

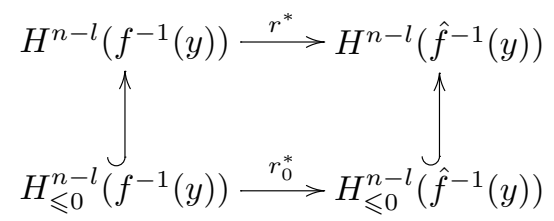

Then $r_{0}^{*}$ is an injection of pure Hodge structures and we have a splitting injection of local systems

$$
L_{0, l, S}^{f} \rightarrow L_{0, l, S}^{\hat{f}} .
$$

Proof. - Let

$$
\widehat{Z}_{S} \stackrel{\rho}{\rightarrow} \overline{\hat{f}^{-1}(S)}
$$

be any projective and surjective map from a nonsingular space and $\theta:=\widehat{Z}_{S} \rightarrow \bar{S}$ be the resulting map.

We may assume, by refining the stratification if necessary, that $\theta$ is smooth over $S$.

We have the commutative diagram

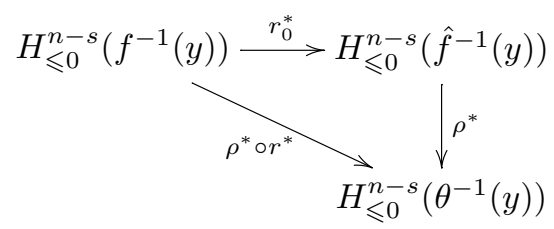

where $\rho^{*} \circ r^{*}$ is injective by the same argument as in the proof of the Theorem 2.2.1.

It follows that $r_{0}^{*}$ is injective. The existence of a splitting comes from semisimplicity. 
Proof of Theorem 2.2.3(a). - There is a commutative diagram

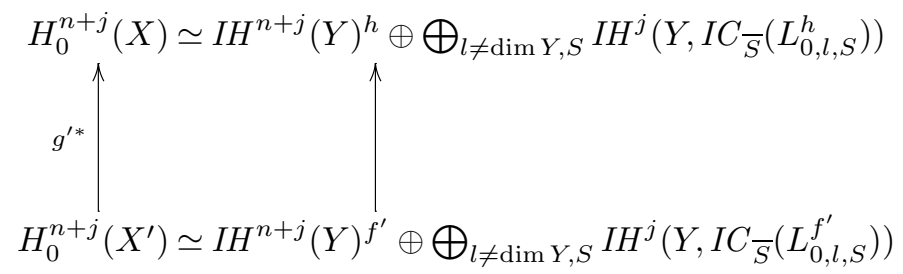

where, by 3.8.2, the vertical map on the right is direct sum map of maps of pure Hodge structures identifying the pure Hodge structure on $I H(Y)^{h}$ with the one on $I H(Y)^{\hat{f}^{\prime}}(Y)$.

Proof of Theorem 2.2.3(b). - By transversality, the complex $r^{*} I C_{Y}[-1]$ satisfies the conditions characterizing $I C_{Y_{1}}$ (cf. 3.8). The result follows from Proposition 4.7.7.

Proof of Theorem 2.2.3(c). - Let $f: X \rightarrow Y$ be a projective resolution of the singularities of $Y$. By the Semisimplicity Theorem 2.1.1(c), the complex $I C_{Y}$ is a direct summand of ${ }^{p} \mathcal{H}^{0}\left(f_{*} \mathbb{Q}_{X}[\operatorname{dim} X]\right)$. By Remark 4.4.3, the cup product map $A^{j}$ is a direct sum map. By Theorem 2.1.4, recalling that we are identifying $L^{j}$ with $A^{j}$, the map $A^{j}$ is an isomorphism on every direct summand, whence the Hard Lefschetz-type statement and its standard algebraic consequence, i.e. the primitive Lefschetz Decomposition.

Proof of Theorem 2.2.3(d). - Since $f$ is birational, (i) the complexes ${ }^{p} \mathcal{H}^{i}\left(f_{*} \mathbb{Q}_{X}[n]\right)$ are supported on proper closed algebraic subsets of $Y$ for every $i \neq 0$ and (ii) $I C_{Y}$ is a direct summand of $\operatorname{Ker} \eta=\mathcal{P}_{\eta}^{0} \subseteq{ }^{\mathcal{H}} \mathcal{H}^{0}\left(f_{*} \mathbb{Q}_{X}[\operatorname{dim} X]\right)$ and is the only summand supported on $Y$.

Let $\eta$ be any ample line bundle on $X$. The result follows from the Generalized HodgeRiemann Bilinear Relations 2.1.8, 2.2.3(a), Remark 3.1.1 and from the fact that $L$ acts compatibly with any direct sum decomposition by Remark 4.4.3.

\section{Acknowledgements}

The authors would like to thank Victor Ginzburg, Mark Goresky, Agnes Szilard, Arpad Toth and Dror Varolin for useful remarks. They would like to thank Robert MacPherson for suggesting improvements and making useful remarks. The first-named author would like to thank the following institutions for their kind hospitality while portions of this work were being carried out: the Korean Institute for Advanced Study, Seoul, the Institute for Advanced Study, Princeton, the Mathematical Sciences Research Institute, Berkeley, the Max Planck Institut für Mathematik, Bonn and the Department of Mathematics of the University of Bologna. The second-named author would like to thank the Department of Mathematics of SUNY at Stony Brook and the Department of Mathematics of the University of Rome, "La Sapienza."

The first-named author dedicates this paper to his family and to the memory of Meeyoung Kim.

The second-named author dedicates this paper to his father for his eightieth birthday, and to E., F. and G.

$4^{\mathrm{e}}$ SÉRIE - TOME $38-2005-\mathrm{N}^{\circ} 5$ 


\section{REFERENCES}

[1] Beilinson A.A., Bernstein J.N., Deligne P., Faisceaux pervers, Astérisque, vol. 100, Soc. Math. France, Paris, 1982.

[2] Borel A., et al., Intersection Cohomology, Progr. Math., vol. 50, Birkhäuser, Boston, 1984.

[3] Borho W., MacPherson R., Partial resolutions of nilpotent varieties, Astérisque 101-102 (1983) 23-74.

[4] Cattani E., Kaplan A., Schmid W., $L^{2}$ and intersection cohomologies for a polarizable variation of Hodge structure, Invent. Math. 87 (1987) 217-252.

[5] Clemens C.H., Degeneration of Kähler manifolds, Duke Math. J. 44 (2) (1977) 215-290.

[6] de Cataldo M., Migliorini L., The Hard Lefschetz theorem and the topology of semismall maps, Ann. Sci. École Norm. Sup. (4) 35 (5) (2002) 759-772.

[7] de Cataldo M., Migliorini L., The Chow Motive of semismall resolutions, Math. Res. Lett. 11 (2004) 151-170.

[8] de Cataldo M., Migliorini L., The Hodge theory of algebraic maps, math.AG/0306030.

[9] Deligne P., Théorème de Lefschetz et critères de dégénérescence de suites spectrales, Publ. Math. IHÉS 35 (1969) 107-126.

[10] Deligne P., Décompositions dans la catégorie dérivée, in: Motives, Seattle, WA, 1991, Part 1, in: Proc. Sympos. Pure Math., vol. 55, American Mathematical Society, Providence, RI, 1994, pp. 115-128.

[11] Deligne P., Théorie de Hodge, II, Publ. Math. IHÉS 40 (1971) 5-57.

[12] Deligne P., Théorie de Hodge, III, Publ. Math. IHÉS 44 (1974) 5-78.

[13] Deligne P., La conjecture de Weil, II, Publ. Math. IHÉS 52 (1980) 138-252.

[14] Dimca A., Sheaves in Topology, Universitext, Springer, Berlin, 2004.

[15] El Zein F., Théorie de Hodge des cycles évanescents, Ann. Sci. École Norm. Sup. (4) 19 (1986) $107-184$.

[16] Goresky M., MaCPherson R., Intersection homology II, Invent. Math. 71 (1983) 77-129.

[17] Goresky M., MacPherson R., Stratified Morse Theory, Ergeb. Math. (3), vol. 2, Springer, Berlin, 1988.

[18] Guillen F., Navarro Aznar V., Sur le théorème local des cycles invariants, Duke Math. J. 61 (1) (1990) 133-155.

[19] IVERSEN B., Cohomology of Sheaves, Universitext, Springer, Berlin, 1986.

[20] Kashiwara M., Kawai T., The Poincaré lemma for variations of polarized Hodge structures, Publ. Res. Inst. Math. Sci. 23 (1987) 345-407.

[21] Kashiwara M., Schapira P., Sheaves on Manifolds, Grundlehren Math. Wiss., vol. 292, Springer, Berlin, 1990.

[22] R. MaCPherson, Global questions in the topology of singular spaces, in: Proc. of the ICM, 1983, Warszawa, pp. 213-235.

[23] Mochizuki T., Asymptotic behaviour of tame nilpotent harmonic bundles with trivial parabolic structure, J. Differential Geom. 62 (2002) 351-559.

[24] Mochizuki T., Asymptotic behaviour of tame harmonic bundles and an application to pure twistor D-modules, math.DG/0312230, 2003.

[25] C. SAвван, Polarizable twistor D-modules, Preprint.

[26] Saito M., Modules de Hodge polarisables, Publ. Res. Inst. Math. Sci. 24 (6) (1988) 849-995.

[27] Saito M., Mixed Hodge modules, Publ. Res. Inst. Math. Sci. 26 (2) (1990) 221-333.

[28] SaIto M., Decomposition theorem for proper Kähler morphisms, Tohoku Math. J. (2) 42 (2) (1990) 127-147.

[29] Sommese A.J., Submanifolds of Abelian varieties, Math. Ann. 233 (3) (1978) 229-256.

[30] STEEnbRInK J., Limits of Hodge structures, Invent. Math. 31 (1975-1976) 229-257. 
[31] Steendirink J., Zucker S., Variation of mixed Hodge structure. I, Invent. Math. 80 (3) (1985) 489542.

[32] VoIsin C., Théorie de Hodge et géométrie algébrique complexe, Cours spécialisés, vol. 10, Société Mathématique de France, Paris, 2002.

(Manuscrit reçu le 19 mai 2004;

accepté, après révision, le 8 juillet 2005.)

Mark Andrea A. DE CATALDO

Department of Mathematics,

SUNY at Stony Brook,

Stony Brook, NY 11794, USA

E-mail: mde@math.sunysb.edu

Luca MIGLIORINI

Dipartimento di Matematica,

Università di Bologna,

Piazza di Porta S. Donato 5,

40126 Bologna, Italy

E-mail:migliori@dm.unibo.it

$4^{\mathrm{e}}$ SÉRIE - TOME $38-2005-\mathrm{N}^{\circ} 5$ 